\title{
Counting the number of Killing vectors in a 3D spacetime
}

\author{
Masato Nozawa \\ Center for Gravitational Physics, Yukawa Institute for Theoretical Physics, Kyoto University, \\ Kyoto 606-8502, Japan \\ E-mail: masato.nozawa@yukawa.kyoto-u.ac.jp
}

\author{
Kentaro Tomoda \\ Advanced Mathematical Institute, Osaka City University, Osaka 558-8585 Japan \\ E-mail: k-tomoda@sci.osaka-cu.ac.jp
}

\begin{abstract}
We devise an algorithm which allows one to count the number of Killing vectors for a Lorentzian manifold of dimension 3. Our algorithm relies on the principal traces of powers of the Ricci tensor and branches intricately according to the values of differential invariants arising from the compatibility conditions of the Killing equation. As illustrating examples, we classify the Lifshitz and pp-wave spacetimes into a hierarchy based on their level of symmetry. A complete classification of spacetimes admitting 4 Killing vectors is also presented.

PACS numbers: 04.20.-q, 02.40.-k
\end{abstract}

Keywords: Killing vector, isometry group, differential invariant, Cartan scalar, CartanKarlhede algorithm, vanishing scalar invariant space

\section{Introduction and summary of our work}

Klein's Erlangen programme [1] provided an attempt to make a connection between geometry and symmetry. In modern parlance, this programme tried to classify the geometry according to the invariant properties under a certain (finite) group action. Whilst this paradigm has turned out to be too narrow to encompass the Riemannian geometry, it nevertheless has significant implications and influences to Riemannian geometry, as well as for theoretical physics. The advent of general relativity stimulated Klein to investigate the role of groups from relativistic standpoint. He had regarded special relativity as the theory of invariants of Minkowski spacetime under the Lorentz group action and also contributed to the formulation of the conservation laws in general relativity, see [2] for a review.

An isometry group acting on a Lorentzian manifold $\left(M, g_{a b}\right)$ is of fundamental importance in understanding the geometrical and physical properties of a spacetime. For instance, the isometry group allows one to set the privileged local coordinate system of $M$ and 


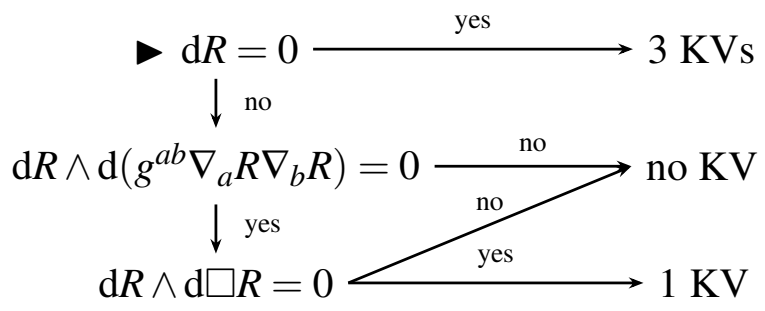

Figure 1a. The Darboux algorithm for a (semi-)Riemannian space of dimension 2. Here $R$ denotes the Ricci scalar; $\nabla_{a}$ denotes the Levi-Civita connection; a box denotes d'Alembertian, $\square \equiv g^{a b} \nabla_{a} \nabla_{b}$; a triangle symbol $>$ stands for a root of this algorithm.

also gives rise to first integrals of geodesic flow that are linear in momenta. In particular, the globally conserved quantities associated with isometries-such as the mass and the angular momentum-play a significant role in the analysis of black holes. It is then natural to ask whether there exists a set of invariants in Klein's sense which is associated with the isometry group.

In this paper, we focus on the local existence of infinitesimal isometries of $\left(M, g_{a b}\right)$. The problem of finding all of them is equivalent to that of finding all independent Killing vectors (KVs). To give an exhaustive list of KVs for a given Riemannian/Lorentzian manifold has a long history in geometry, which dates back at least to Darboux [3]. For dimension $n=2$, he found a set of invariants for determining the existence and the number of KVs as shown in Figure 1a. See also [4, 5, 6, 7, 8] for pertinent results.

For determining the number of KVs in arbitrary dimension, Cartan's equivalence method or the Cartan-Karlhede algorithm [9, 10] is known as an effective technique (see also section 9.2 of [11]). Though it was originally developed to solve alias the equivalence problem, that is the problem of deciding whether given two (semi-)Riemannian manifolds are locally isometric, this amounts to determining the dimension of their local isometry group, as well as the structure constants of the group. Their machinery is explained as follows. Let $\left(M, g_{a b}\right)$ be a (semi-)Riemannian manifold of dimension $n$ and $\mathscr{C}^{p}$ be the set of Cartan scalars of order $p$. Note that Cartan scalars of order $p$ are defined as the frame components of the Riemann-Christoffel tensor and its first $p$ covariant derivatives. As with Newman-Penrose scalars, Cartan scalars are functions on the tangent frame bundle $F(M)$ but not on $M$. Cartan showed that the local geometry of $M$ is completely determined by $\mathscr{C}^{p_{0}}$ with $p_{0} \leq \frac{1}{2} n(n+1)$, where $p_{0}$ is the smallest natural number such that the elements of $\mathscr{C}^{p_{0}+1}$ are functionally dependent on those in $\mathscr{C}^{p_{0}}$. Subsequently, Karlhede demonstrated that in dimension $n=4$ at most 7 differentiations suffice, whereat 3,156 functionally independent scalars are required. Given the set $\mathscr{C}^{p}$ consisting of $q$ functionally independent scalars, the manifold $M$ admits $\frac{1}{2} n(n+1)-q$ independent KVs. Unfortunately, the actual computations required to perform the Cartan-Karlhede algorithm remain formidable, even though it indeed ensures that the problem of finding all KVs is computable.

For a Riemannian space of dimension $n=3$, an important progress has been made in this problem rather recently in [12]. The scheme exploits the compatibility condition of the 
Killing equation (see eq. (1.2) below), and aims exclusively at determining the dimension of the isometry group. This provides a more efficient and effective algorithm to count the number of KVs, allowing us to circumvent enormous amount of computational efforts. We discuss in this paper its extension to a Lorentzian manifold $\left(M, g_{a b}\right)$ of dimension 3. An essential ingredient which operates the mechanism is as follows: Recall that any vector $K^{a}$ is a $\mathrm{KV}$ on $\left(M, g_{a b}\right)$ if and only if the Killing equation is satisfied

$$
£_{K} g_{a b}=2 \nabla_{(a} K_{b)}=0 .
$$

Here $£_{K}$ is the Lie derivative along $K^{a}, \nabla_{a}$ denotes the Levi-Civita connection and indices are raised and lowered with $g_{a b}$ and its inverse. The round brackets denote symmetrisation over the enclosed indices. As the compatibility condition of eq. (1.1), one finds the curvature collineation [13]

$$
£_{K} R_{a b c}{ }^{d}=0,
$$

where $R_{a b c}{ }^{d}$ is the Riemann-Christoffel tensor defined by $2 \nabla_{[a} \nabla_{b]} V_{c}=R_{a b c}{ }^{d} V_{d}$. Here the square brackets over indices is used for skew-symmetrisation. Any solution to the Killing equation (1.1) automatically solves the equation (1.2), but in general the converse is not true. In dimension 3, the following condition is an immediate corollary of (1.2):

$$
£_{K} R=0, \quad £_{K} S^{(2)}=0, \quad £_{K} S^{(3)}=0,
$$

where $R_{a b} \equiv R_{a c b}{ }^{c}$ is the Ricci tensor, $R \equiv R_{a}^{a}=g^{a b} R_{a b}$ is the scalar curvature, and $S^{(2)} \equiv S^{a}{ }_{b} S^{b}{ }_{a}, S^{(3)} \equiv S^{a}{ }_{b} S^{b}{ }_{c} S^{c}{ }_{a}$ are the principal traces of powers of the traceless Ricci tensor $S_{a b} \equiv R_{a b}-(R / 3) g_{a b}$. Thus, any solution to eq. (1.1) must satisfy the following matrix equation $\neq$

$$
\boldsymbol{R}_{a} K^{a}=0, \quad \boldsymbol{R}_{a} \equiv\left[\begin{array}{c}
\nabla_{a} R \\
\nabla_{a} S^{(2)} \\
\nabla_{a} S^{(3)}
\end{array}\right] .
$$

We shall refer to the $3 \times 3$ matrix $\boldsymbol{R}_{a}$ as the first obstruction matrix. This equation implies that any KV must be in $\operatorname{ker} \boldsymbol{R}_{a}$ and hence $\operatorname{det} \boldsymbol{R}_{a}=0$ for $\operatorname{ker} \boldsymbol{R}_{a} \neq \emptyset$, where the determinant of $\boldsymbol{R}_{a}$ is given by

$$
\operatorname{det} \boldsymbol{R}_{a}=\mathrm{d} R \wedge \mathrm{d} S^{(2)} \wedge \mathrm{d} S^{(3)} .
$$

The first obstruction matrix $\boldsymbol{R}_{a}$ is classified by the dimension of its kernel,

$$
d \equiv \operatorname{dim} \operatorname{ker} \boldsymbol{R}_{a}=3-\operatorname{rank} \boldsymbol{R}_{a},
$$

which can be determined according to the minors of $\boldsymbol{R}_{a}$ :

$$
\begin{array}{lll}
\mathrm{d} R \wedge \mathrm{d} S^{(2)}, & \mathrm{d} S^{(2)} \wedge \mathrm{d} S^{(3)}, & \mathrm{d} S^{(3)} \wedge \mathrm{d} R, \\
\mathrm{~d} R, & \mathrm{~d} S^{(2)}, & \mathrm{d} S^{(3)} .
\end{array}
$$

\$ The terms $S^{(m)}(m \geq 4)$ fail to contribute to the obstruction matrix, since the Cayley-Hamilton theorem allows one to express them as lower matrix powers. 
It follows that $\left(M, g_{a b}\right)$ enjoys a local isometry group of dimension $d_{\text {iso. }} \leq \frac{1}{2} d(d+1)$ with an isotropy subgroup of dimension $d_{\text {sub. }} \leq \frac{1}{2} d(d-1)$, acting on orbits of dimension $d_{\text {orb. }} \leq d$.

In any of these cases, a general solution of eq. (1.4) can be written in the form

$$
K^{a}=\sum_{\alpha} \omega_{\alpha} u_{\alpha}^{a}, \quad(\alpha=1, \ldots, d)
$$

where $\left\{u_{\alpha}^{a}\right\}$ are linearly independent vectors that span $\operatorname{ker} \boldsymbol{R}_{a}$ and the coefficients $\left\{\omega_{\alpha}\right\}$ are left arbitrary. In what follows, we refer to the case in which eq. (1.8) holds true as class $d$. Substituting the form (1.8) into eq. (1.1), we obtain a PDE system of the form

$$
\nabla_{a} \boldsymbol{\omega}=\boldsymbol{\Omega}_{a} \boldsymbol{\omega}, \quad \boldsymbol{\omega} \equiv\left[\begin{array}{ll}
\omega_{\alpha} & \varpi_{\beta}
\end{array}\right]^{T}, \quad\left(\beta=1, \ldots, m \equiv \frac{1}{2} d(d-1)\right)
$$

where $\left\{\varpi_{\beta}\right\}$ are the 1-jet variables and the connection $\boldsymbol{\Omega}_{a}$ is expressed in terms of the Ricci rotation coefficients and their ratio. Since eq. (1.9) is the first-order system, its compatibility gives rise to algebraic constraints on $\boldsymbol{\omega}$,

$$
\left(\nabla_{[a} \boldsymbol{\Omega}_{b]}-\boldsymbol{\Omega}_{[a} \boldsymbol{\Omega}_{b]}\right) \boldsymbol{\omega}=0 .
$$

Equivalently, eq. (1.10) can be written in component form of curvature of the bundle

$$
\boldsymbol{R}_{\mathrm{cls} . d} \boldsymbol{\omega}=0 .
$$

We henceforth call $\boldsymbol{R}_{\mathrm{cls} . d}$ as the second obstruction matrix of class $d$. Since the matrix equation (1.4) is a necessary condition for eq. (1.1), the second obstruction matrix yields obstructions to the existence of KVs as differential invariants. A noteworthy asset of this method is that the obstruction is measured by a purely algebraic fashion.

Let us now outline our strategy to be carried out. In each class $d$, we solve eq. (1.10) and then update the form (1.8). When this achives a decrease in the number $d$ in eq. (1.8) or in the number $m$ in eq. (1.9), which are initially given by $d=\operatorname{dim} \operatorname{ker} \boldsymbol{R}_{a}$ and $m=\frac{1}{2} d(d-1)$ respectively, we write out eqs. (1.9)-(1.11) with the updated form, that is the form (1.8) with $\boldsymbol{\omega}$ being the solution of eq. (1.10). Once again, we solve eq. (1.10) and re-update the form (1.8). We iterate this procedure until the latest compatibility is met trivially, or until the number $d$ vanishes as a consequence thereof. This procedure is amendable to a follow-up study. This is a prime advantage of our formulation over the treatment of Cartan-Karlhede.

In this paper, we classify the number of local isometry group for a Lorentzian manifold of dimension 3, by presenting the explicit forms of the second obstruction matrix $\boldsymbol{R}_{\text {cls. } d}$ in all classes. This survey is essentially based on the procedure developed in [12], but differs from it in that: In Lorentzian signature, there appear null KVs and the Ricci tensor is not always diagonalisable. It is this aspect that prohibits the direct application of the previous analysis of Riemannian case [12] and requires the separate study, complicating attempts to pin it down discursively. The strategy employed here is similar in spirit to the Erlangen programme, since the symmetry is classified in terms of differential invariants. On top of the intrinsic interest in 3 spacetime dimensions, the method developed here can be applicable also 
for the 3 dimensional induced metrics as well as for quotient metrics. For $n \geq 4$ dimensions, a considerable number of loose ends are left over and the study of counting KVs remains open. See e.g. [14] for the analysis giving rise to an upper bound of KVs. We hope to address the issue for $n \geq 4$ in the future.

Our main results in this paper can be summarised as follows:

Theorem. Let $\left(M, g_{a b}\right)$ be a 3-dimensional Lorentzian manifold. The number of linearly independent Killing vectors is counted by an algorithm described in Figure 1b. It includes sub-algorithms given in Figures $2 a-4 a$.

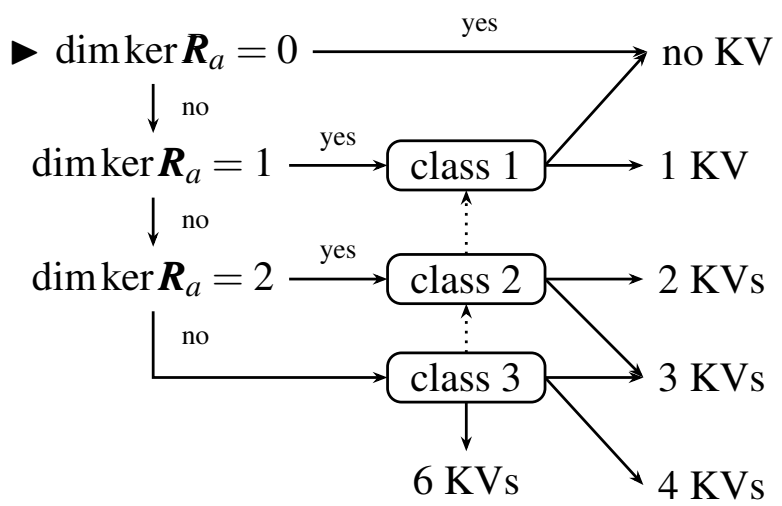

Figure 1b. Main algorithm to determine the number of linearly independent KVs for a 3dimensional spacetime. It consists of the three criteria [see eqs. (1.7)] and the 3-round blocks. The blocks include sub-algorithms which are respectively given in Figures $2 \mathrm{a}-4 \mathrm{a}$. It has the nest structures indicated by dotted lines. By connecting a path which is possibly a combination of solid and dotted lines, the number of KVs is exactly counted. Note that the case admitting $5 \mathrm{KVs}$ is rigid, in the sense that it inevitably generates 6th KV (see e.g. [15] and references therein).

It is noteworthy that the algorithm shown in Figure 1b has the nest structures: The subalgorithm for the class 2 contains that for the class 1 as sub-sub-algorithms; Similarly, the sub-algorithm for the class 3 contains not just that for the class 1 but also that for the class 2 as sub-sub-algorithms. As we will see in Sections 3 and 4, such structures serve to avoid unnecessary repetition and to simplify the whole algorithm.

The rest of this paper is organised as follows. In Sections 2-4, we analyse the Killing equation in classes 1 to 3 in sequence. The corresponding obstruction matrices and subalgorithm are given explicitly. We enlighten readers about the power of our algorithm with some instructive examples in Section 5. We first inspect the Lifshitz spacetime admitting a single parameter $z$, the value of which controls the number of local isometry. After this simple exercise, a special attention is payed to the plane-fronted wave with parallel rays (pp-wave), which admits a covariantly constant null vector and vanishing scalar invariants. This metric epitomises the Lorentzian signature and is specified by a single function. We provide the complete classification of local isometry which turns out to be controlled by the profile of this function. We close this paper with some comments in Section 6. Technical formulae are summarised in Appendix A. An exhaustive classification of spacetimes admitting $4 \mathrm{KVs}$ is 
given in Appendix B. This also serves as an insightful guide to confirm the vindication of the present paper.

Remark that we shall use the same symbol for different sections and subsections recurrently in order to minimise the number of symbols and to lighten the notation. We caution the readers not to be confused by this abuse of notation.

\section{Analysis of class 1}

Let us begin our analysis with the class 1 , in which any $\mathrm{KV}$ can be written as

$$
K^{a} \propto u^{a}
$$

where $u^{a}$ is a vector that annihilates $\boldsymbol{R}_{a}$. The annihilator $u^{a}$ must be specified beforehand, but the results in this section does not depend on the explicit form of $u^{a}$.

For $\operatorname{dim} \operatorname{ker} \boldsymbol{R}_{a}=1$, there is at least one non-vanishing 2-form in eq. (1.7a), which allows us to take $u^{a}$ to be the Hodge dual of it. For instance, $u^{a} \equiv \varepsilon^{a b c} \nabla_{b} R \nabla_{c} S^{(2)}$ for $\mathrm{d} R \wedge \mathrm{d} S^{(2)} \neq 0$, where $\varepsilon^{a b c}$ is the Levi-Civita tensor.

Our discussion has two offshoots according to whether $u^{a}$ is timelike or spacelike (Subsection 2.1), whilst $u^{a}$ is null (Subsection 2.2). Subsection 2.3 gives short summary of this section.

\subsection{Non-null case}

When $u^{a}$ is timelike or spacelike, we can normalise an annihilator of $\boldsymbol{R}_{a}$ to unity by setting

$$
e^{a} \equiv \frac{u^{a}}{\sqrt{\lg _{b c} u^{b} u^{c}}}, \quad g_{a b} e^{a} e^{b}=\imath
$$

where $\imath \equiv \operatorname{sgn}\left(g_{a b} u^{a} u^{b}\right)$. We also introduce the tensor

$$
h_{a b}(e) \equiv g_{a b}-\imath e_{a} e_{b},
$$

that is endowed with a projection property and an orthogonality

$$
h^{a} h_{b}^{c}=h_{b}^{a}, \quad h_{a b} e^{b}=0 .
$$

In this case any KV takes the form

$$
K^{a}=\omega e^{a}
$$

where $\omega$ is an unknown scalar. By using the projection tensor (2.3) and the form (2.5), one can boil down each component of eq. (1.1) to

$$
\begin{aligned}
& 0=e^{a} e^{b} \nabla_{(a} K_{b)}=\imath £_{e} \omega, \\
& 0=e^{a} h^{b}{ }_{c} \nabla_{(a} K_{b)}=\frac{1}{2}\left(\imath \nabla_{c} \omega-e_{c} £_{e} \omega-\imath \Omega_{c}^{\imath} \omega\right), \\
& 0=h^{a}{ }_{c} h^{b}{ }_{d} \nabla_{(a} K_{b)}=\omega \kappa_{c d},
\end{aligned}
$$


where

$$
\Omega_{a}^{l}(e) \equiv-\imath e^{b} \nabla_{b} e_{a}, \quad \quad \kappa_{a b}(e) \equiv h^{c}{ }_{a} h_{b}{ }_{b} \nabla_{(c} e_{d)} .
$$

It follows that the Killing equation (1.1) amounts to

$$
\kappa_{a b}=0, \quad \nabla_{a} \omega=\Omega_{a}^{l} \omega .
$$

It is noted that the condition (2.6a) follows from the second equation in (2.8). The compatibility condition of the latter equation reads $\nabla_{[a} \Omega_{b]}^{l}=0$.

As a result, the necessary and sufficient conditions for the local solvability of eq. (1.1) are aggregated into an algebraic equation

$$
\left(\boldsymbol{R}_{\text {cls. } 1}^{l}\right)_{a b}=0, \quad\left(\boldsymbol{R}_{\text {cls. } 1}^{l}\right)_{a b} \equiv\left[\begin{array}{c}
\kappa_{a b} \\
\nabla_{[a} \Omega_{b]}^{l}
\end{array}\right],
$$

yielding tests for $e^{a}$. If the equation (2.9) is satisfied, there are no extra obstructions for the existence of the Killing vector. This means that precisely one KV exists. On the other hand, the failure of (2.9) means that there exist no KVs.

\subsection{Null case}

In this case, we directly write the $\mathrm{KV}$ as

$$
K^{a}=\omega u^{a}
$$

where $\omega$ is an unknown scalar, keeping the same notation in Subsection 2.1. We also define the projection tensor

$$
q_{a b}(u, v) \equiv g_{a b}-u_{a} v_{b}-v_{a} u_{b},
$$

where $v^{a}$ is a vector filed satisfying $g_{a b} v^{a} v^{b}=0$ and $g_{a b} v^{a} u^{b}=1 \S$. The tensor (2.11) is projective and orthogonal

$$
q_{c}^{a}{ }_{c} q_{b}^{c}=q_{b}^{a}, \quad q_{b}^{a} u^{b}=0, \quad q_{b}^{a} v^{b}=0 .
$$

With the help of eqs. (2.10) and (2.11), the components of eq. (1.1) can be written as follows:

$$
\begin{aligned}
& 0=v^{a} v^{b} \nabla_{(a} K_{b)}=\omega v^{a} v^{b} \nabla_{a} u_{b}+£_{v} \omega, \\
& 0=u^{a} v^{b} \nabla_{(a} K_{b)}=\frac{1}{2}\left(\omega u^{a} v^{b} \nabla_{a} u_{b}+£_{u} \omega\right), \\
& 0=u^{a} q^{b}{ }_{c} \nabla_{(a} K_{b)}=\frac{1}{2} \omega \theta_{c}, \\
& 0=v^{a} q^{b}{ }_{c} \nabla_{(a} K_{b)}=q^{b}{ }_{c}\left(\omega v^{a} \nabla_{(a} u_{b)}+\frac{1}{2} \nabla_{b} \omega\right), \\
& 0=q^{a}{ }_{c} q^{b}{ }_{d} \nabla_{(a} K_{b)}=\omega \kappa q_{c d},
\end{aligned}
$$

$\S$ Remark that the two conditions do not determine $v^{a}$ uniquely. We need to make a particular choice of $v^{a}$ in $M$ [a section of a frame bundle $F(M)$ ]. In spite of this ambiguity, the final outcomes are insensitive to the choice of $v^{a}$. 
where we remark that the $u u$-component is satisfied automatically and the "shear term" in (2.13e) identically vanishes since $q_{a b}$ admits only a single nonvanishing component. Here we have introduced

$$
\kappa(u, v) \equiv q^{a b} \nabla_{a} u_{b}, \quad \theta_{a}(u, v) \equiv u^{b} \nabla_{b} u_{a}-\left(u^{b} v^{c} \nabla_{b} u_{c}\right) u_{a} .
$$

From above equations, it follows that the satisfaction of the Killing equation is tantamount to

$$
\kappa=0, \quad \theta_{a}=0, \quad \nabla_{a} \omega=\Omega_{a} \omega,
$$

where

$$
\Omega_{a}(u, v) \equiv-2 v^{b} \nabla_{(a} u_{b)}+u_{a} v^{b} v^{c} \nabla_{b} u_{c} .
$$

The compatibility condition of the third equation in (2.15) reads $\nabla_{[a} \Omega_{b]}=0$.

As a result, the necessary and sufficient conditions for the local solvability of eq. (1.1) are aggregated into an algebraic equation

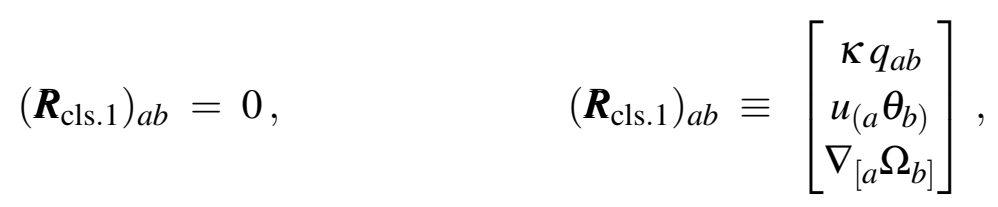

yielding tests for $u^{a}$ and $v^{a}$. If the equation (2.17) is satisfied, there are no extra conditions to be satisfied. Hence, one null KV exists.

\subsection{Short summary of class 1}

We summarise the results here in Figure 2a.

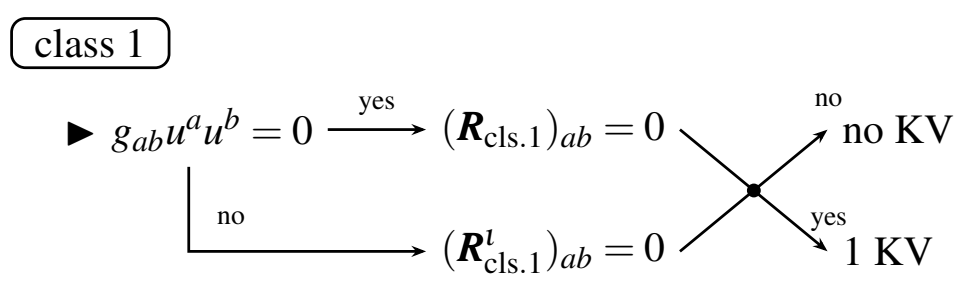

Figure 2a. The sub-algorithm for the class $1, K^{a} \propto u^{a}$. See eqs. (2.9) and (2.17) for notations.

It deserves to emphasise that the sub-algorithm in Figure 2a is applicable also for some cases in class 2 and 3 as explained in Section 1: It might be seemingly appreciated that the prescription in class 2 and 3 is not be adaptive to class 1 , since KVs in either case are expressed as a linear combination of two (or three) annihilators of $\boldsymbol{R}_{a}$ with $\operatorname{dim} \operatorname{ker} \boldsymbol{R}_{a}>1$. Nevertheless, an essential terminus a quo for the argument in class 1 is the assumption $K^{a} \propto u^{a}$ in (2.1), rather than $\operatorname{dim} \operatorname{ker} \boldsymbol{R}_{a}=1$. Indeed, the situation we shall encounter in class 2 and 3 is that the KVs in several branches are proportional to an annihilator of $\boldsymbol{R}_{a}$, whereas $\operatorname{dim} \operatorname{ker} \boldsymbol{R}_{a}>1$. Since $K^{a} \propto u^{a}$ conforms to the applicability of class 1 , no harm is caused in pretending that the recipe in this section is reusable also for such branches. The recyclability of the analysis significantly reduces the total amount of calculations. 


\section{Analysis of class 2}

Our focus in this section is centred on the class 2, in which any $\mathrm{KV}$ can be written as

$$
K^{a}=\sum_{\alpha=1}^{2} \omega_{\alpha} u_{\alpha}^{a},
$$

where $\left\{u_{\alpha}^{a}\right\}$ are vectors that annihilates $\boldsymbol{R}_{a}$. The annihilators must be specified beforehand, but the results in this section do not depend on their explicit form.

For $\operatorname{dim} \operatorname{ker} \boldsymbol{R}_{a}=2$, there is only one linearly independent 1-form in eq. (1.7b), say $u_{a} \|$. It turns out that any vector orthogonal to $u^{a}=g^{a b} u_{b}$ is an annihilator of $\boldsymbol{R}_{a}$. In particular, $u^{a}$ itself is the annihilator if it is null, for which a special handling is required.

We are proceeding along two cases where Subsection 3.1 treats the case where the two annihilators are both non-null, whilst Subsection 2.2 discusses either of them is null. We do not try to examine the case in which two annihilators are null and non-parallel, since we can bring this case to the non-null case by a suitable change of basis. If Subsection 3.3 gives short summary of this section.

\subsection{Non-null case}

In this case, it is assumed that $\left\{e_{i}^{a}, i=1,2,3\right\}$ forms an orthonormal basis of $T(M)$,

$$
g^{a b}=\imath e_{1}^{a} e_{1}^{b}+e_{2}^{a} e_{2}^{b}-\imath e_{3}^{a} e_{3}^{b},
$$

where $\imath \equiv \operatorname{sgn}\left(g_{a b} e_{1}^{a} e_{1}^{b}\right)$, and two vectors $\left\{e_{2}^{a}, e_{3}^{a}\right\}$ are two annihilators of $\boldsymbol{R}_{a}$. Remark that $e_{2}^{a}$ is spacelike, whereas $e_{3}^{a}$ is either spacelike for $\imath=-1$ or timelike for $\imath=+1$.

For $\operatorname{dim} \operatorname{ker} \boldsymbol{R}_{a}=2$, the basis $\left\{e_{i}^{a}\right\}$ is taken as follows: One can choose $e_{1}^{a}$ in such a way that it is proportional to $u^{a}$, i.e.

$$
e_{1}^{a} \equiv \frac{u^{a}}{\sqrt{\iota_{u} g_{b c} u^{b} u^{c}}}
$$

where $\imath_{u} \equiv \operatorname{sgn}\left(g_{a b} u^{a} u^{b}\right)$. So $e_{2}^{a}$ can be taken as a vector such that $g_{a b} e_{1}^{a} e_{2}^{b}=0$ and $g_{a b} e_{2}^{a} e_{2}^{b}=1,{ }^{+}$in terms of which one can specify $e_{3}^{a}$ to be $e_{3}^{a} \equiv \varepsilon^{a b c} e_{1 b} e_{2 c}$.

Given these orthogonal frame $\left\{e_{i}^{a}\right\}$, any KV can be written in the form

$$
K^{a}=\omega_{2} e_{2}^{a}+\omega_{3} e_{3}^{a},
$$

where scalars $\left\{\omega_{2}, \omega_{3}\right\}$ are yet indeterminate.

\| To reduce the number of symbols needed, we have employed the same symbol as that in Section 2. The reader is cautioned not to confuse it with eq. (2.1).

II In an arbitrary dimensional Lorentzian manifold, two null vectors orthogonal to each other must be proportional. This fact allows us to exclude this possibility.

+ Similar to $v^{a}$ in Subsection 2.2 the two conditions do not determine $e_{2}^{a}$ uniquely. We need to make a particular choice of $e_{2}^{a}$ in $M$. Again, the results here do not depend on the choice of $e_{2}^{a}$. 
Instead of writing down the components of eq. (1.1) with eq. (3.4) by the coordinate basis, it is much more convenient to work with the connection components. For this purpose, let us introduce the Ricci rotation coefficients as

$$
\begin{aligned}
e_{1}^{b} \nabla_{b}\left[\begin{array}{l}
e_{1}^{a} \\
e_{2}^{a} \\
e_{3}^{a}
\end{array}\right] & =\left[\begin{array}{ccc}
0 & \kappa_{1} & -\imath \eta_{1} \\
-\imath \kappa_{1} & 0 & -\imath \tau_{1} \\
-\imath \eta_{1} & -\tau_{1} & 0
\end{array}\right]\left[\begin{array}{l}
e_{1}^{a} \\
e_{2}^{a} \\
e_{3}^{a}
\end{array}\right], \\
e_{2}^{b} \nabla_{b}\left[\begin{array}{l}
e_{1}^{a} \\
e_{2}^{a} \\
e_{3}^{a}
\end{array}\right] & =\left[\begin{array}{ccc}
0 & -\kappa_{2} & -\imath \tau_{2} \\
\imath \kappa_{2} & 0 & -\imath \eta_{2} \\
-\imath \tau_{2} & -\eta_{2} & 0
\end{array}\right]\left[\begin{array}{l}
e_{1}^{a} \\
e_{2}^{a} \\
e_{3}^{a}
\end{array}\right], \\
e_{3}^{b} \nabla_{b}\left[\begin{array}{c}
e_{1}^{a} \\
e_{2}^{a} \\
e_{3}^{a}
\end{array}\right] & =\left[\begin{array}{ccc}
0 & \tau_{3} & \imath \kappa_{3} \\
-\imath \tau_{3} & 0 & \imath \eta_{3} \\
\imath \kappa_{3} & \eta_{3} & 0
\end{array}\right]\left[\begin{array}{l}
e_{1}^{a} \\
e_{2}^{a} \\
e_{3}^{a}
\end{array}\right],
\end{aligned}
$$

where $\kappa_{i}, \eta_{i}$, and $\tau_{i}$ are respectively the geodesic curvature, normal curvature and relative torsion of an integral curve of $e_{i}^{a}$. Their derivatives entail relations amongst each other, which are collected in Appendix A.1.

By using eqs. (3.2)-(3.5), the 11-part of eq. (1.1) can be formally boiled down to

$$
\kappa_{1} \omega_{2}+\eta_{1} \omega_{3}=0
$$

yielding tests for $e_{1}^{a}$. This implies that the analysis branches off, depending on whether $e_{1}^{a}$ satisfies the geodesic equation $e_{1}^{b} \nabla_{b} e_{1}^{a}=0$.

3.1.1. Branch where $e_{1}^{a}$ is not a geodesic tangent $\kappa_{1} \eta_{1} \neq 0$ From eq. (3.6), $\omega_{2}$ and $\omega_{3}$ are related to each other, $\omega_{3}=-\left(\kappa_{1} / \eta_{1}\right) \omega_{2}$, or $\omega_{2}=-\left(\eta_{1} / \kappa_{1}\right) \omega_{3}$. This allows us to rewrite eq. (3.4) as

$$
K^{a}=\omega_{2}\left(e_{2}^{a}-\frac{\kappa_{1}}{\eta_{1}} e_{3}^{a}\right), \quad \text { or } \quad K^{a}=\omega_{3}\left(e_{3}^{a}-\frac{\eta_{1}}{\kappa_{1}} e_{2}^{a}\right)
$$

which matches the assumption (2.1) of the class 1. It turns out that the annihilator of $\boldsymbol{R}_{a}$ is specified as $e_{2}^{a}-\left(\kappa_{1} / \eta_{1}\right) e_{3}^{a}$ or $e_{3}^{a}-\left(\eta_{1} / \kappa_{1}\right) e_{2}^{a}$. As explained in 2.3 , the sub-algorithm described in Figure 2a can be immediately testable.

3.1.2. Branch where $e_{1}^{a}$ is a geodesic tangent $\kappa_{1}=\eta_{1}=0 \quad$ In this branch, the 11-part of the Killing equation (3.6) is satisfied automatically. The remaining parts read

$$
\begin{aligned}
& £_{1} \omega_{2}=-\kappa_{2} \omega_{2}+\left(\tau_{1}+\tau_{2}\right) \omega_{3}, \\
& £_{1} \omega_{3}=\imath\left(\tau_{1}-\tau_{3}\right) \omega_{2}+\imath \kappa_{3} \omega_{3}, \\
& £_{2} \omega_{2}=\eta_{2} \omega_{3}, \\
& £_{2} \omega_{3}=\varpi \\
& £_{3} \omega_{2}=-\eta_{2} \omega_{2}-\eta_{3} \omega_{3}+\imath \varpi, \\
& £_{3} \omega_{3}=-\imath \eta_{3} \omega_{2} .
\end{aligned}
$$


where $£_{i}$ denotes the Lie derivative along $e_{i}^{a}$ and eq. (3.8d) is the defining equation of the 1 -jet variable $\varpi$. Since the PDE system (3.8) is not closed with respect to unknown scalars $\left\{\omega_{2}, \omega_{3}, \varpi\right\}$, we need additional relations between them. Such relations come from several parts of the identities $\nabla_{[a} \nabla_{b]} \omega_{2}=\nabla_{[a} \nabla_{b]} \omega_{3}=0$ [c.f. eq. (A.2)], leading to

$$
\begin{aligned}
2\left(\tau_{3}-\tau_{2}\right) \varpi= & -\left(£_{2}\left(\kappa_{2}-\imath \kappa_{3}\right)+\imath £_{3}\left(\tau_{3}-\tau_{2}\right)-2 \imath \eta_{2}\left(\tau_{3}-\tau_{2}\right)\right) \omega_{2} \\
& -\left(£_{2}\left(\tau_{3}-\tau_{2}\right)+£_{3}\left(\kappa_{2}-\imath \kappa_{3}\right)\right) \omega_{3}, \\
2\left(\tau_{3}+\tau_{2}\right) \varpi= & \left(£_{2}\left(\kappa_{2}+\imath \kappa_{3}\right)-\imath £_{3}\left(\tau_{3}-\tau_{2}\right)\right) \omega_{2} \\
& +\left(£_{2}\left(\tau_{3}-\tau_{2}\right)+£_{3}\left(\kappa_{2}+\imath \kappa_{3}\right)+2 \imath \eta_{3}\left(\tau_{3}+\tau_{2}\right)\right) \omega_{3}, \\
\left(\kappa_{2}+\imath \kappa_{3}\right) \varpi= & \imath\left(£_{2} \tau_{3}\right) \omega_{2}+\imath\left(\eta_{3}\left(\kappa_{2}+\imath \kappa_{3}\right)+£_{3} \tau_{2}\right) \omega_{3} .
\end{aligned}
$$

This implies that $\varpi$ can be expressed in terms of $\omega_{2}$ and $\omega_{3}$ except for $\kappa_{2}+\imath \kappa_{3}=\tau_{3}=\tau_{2}=0$. Depending on the nonzeroness of the coefficients $\left\{\kappa_{2}+\imath \kappa_{3}, \tau_{2}+\tau_{3}, \tau_{2}-\tau_{3}\right\}$, the analysis further falls into four sub-branches.

Sub-branch where $\tau_{2}=\tau_{3}=\kappa_{2}+\imath \kappa_{3}=0 \quad$ In this sub-branch, the 1 -jet variable $\varpi$ cannot be expressed in terms of $\omega_{2}$ and $\omega_{3}$. The differential equations for $\varpi$ come from the remaining parts of the identities $\nabla_{[a} \nabla_{b]} \omega_{2}=\nabla_{[a} \nabla_{b]} \omega_{3}=0$. By combining this and eqs. (3.8), we obtain a PDE system

$$
\nabla_{a} \boldsymbol{\omega}=\boldsymbol{\Omega}_{a}^{l} \boldsymbol{\omega}, \quad \boldsymbol{\omega} \equiv\left[\begin{array}{lll}
\omega_{2} & \omega_{3} & \varpi
\end{array}\right]^{T},
$$

where

$$
\begin{gathered}
\boldsymbol{\Omega}_{a}^{\imath} \equiv l e_{1 a}\left[\begin{array}{ccc}
-\kappa_{2} & \tau_{1} & 0 \\
l \tau_{1} & -\kappa_{2} & 0 \\
\imath £_{2} \tau_{1}+\eta_{3} \tau_{1} & \imath \eta_{2} \tau_{1}-£_{2} \kappa_{2} & 0
\end{array}\right]+e_{2 a}\left[\begin{array}{ccc}
0 & \eta_{2} & 0 \\
0 & 0 & 1 \\
\imath £_{2} \eta_{2} & \eta_{3}^{2}+l £_{3} \eta_{2}+l £_{2} \eta_{3} & 0
\end{array}\right] \\
-l e_{3 a}\left[\begin{array}{ccc}
-\eta_{2} & -\eta_{3} & \imath \\
-\imath \eta_{3} & 0 & 0 \\
-\imath £_{2} \eta_{3}-\eta_{3}^{2} & -\imath \eta_{2} \eta_{3} & \eta_{2}
\end{array}\right] .
\end{gathered}
$$

The compatibility condition, $\left(\nabla_{[a} \boldsymbol{\Omega}_{b]}^{l}-\boldsymbol{\Omega}_{[a}^{l} \boldsymbol{\Omega}_{b]}^{l}\right) \boldsymbol{\omega}=0$, for eq. (3.10) reads

$$
\boldsymbol{R}_{\mathrm{cls} .2}^{l} \boldsymbol{\omega}=0, \quad \boldsymbol{R}_{\mathrm{cls} .2}^{l} \equiv\left[\begin{array}{lll}
£_{2} \kappa_{2} & £_{3} \kappa_{2} & 0 \\
£_{2} \lambda_{2} & £_{3} \lambda_{2} & 0
\end{array}\right],
$$

where $\lambda_{2} \equiv R_{a b} e_{2}^{a} e_{2}^{b}$. Remark that the first line corresponds to eq. (3.9a), and some remaining components are derivable from its derivative.

In this sub-branch, the rank of $\boldsymbol{R}_{\text {cls. } 2}^{l}$ controls the number of KVs: If $\operatorname{rank} \boldsymbol{R}_{\text {cls. } 2}^{l}=0$, three $\mathrm{KVs}$ exist; If rank $\boldsymbol{R}_{\mathrm{cls} .2}^{l}=2$, there is no KV; Otherwise $\operatorname{rank} \boldsymbol{R}_{\mathrm{cls} .2}^{l}=1, \omega_{2}$ and $\omega_{3}$ are related to each other. This implies that the KVs in this branch are proportional to an annihilator of $\boldsymbol{R}_{a}$. As explained in 2.3, the sub-algorithm described in Figure 2a can be immediately testable. 
Other sub-branches Except for the case of $\tau_{2}=\tau_{3}=\kappa_{2}+\imath \kappa_{3}=0$, eqs. (3.9) allow us to write the 1 -jet variable $\Phi$ in terms of $\left\{\omega_{2}, \omega_{3}\right\}$. In these sub-branches, eq. (3.8) is closed with respect to $\left\{\omega_{2}, \omega_{3}\right\}$ and then one needs to consider separately the compatibility of PDE systems of the form

$$
\nabla_{a} \boldsymbol{\omega}=\boldsymbol{\Omega}_{a}^{l} \boldsymbol{\omega}, \quad \boldsymbol{\omega} \equiv\left[\begin{array}{ll}
\omega_{2} & \omega_{3}
\end{array}\right]^{T} .
$$

Remark that explicit forms of the connection $\boldsymbol{\Omega}_{a}^{l}$ and the obstruction matrix $\boldsymbol{R}_{\text {cls.2 }}^{l}$ depend on the nonzeroness of the coefficients $\left\{\kappa_{2}+\imath \kappa_{3}, \tau_{2}, \tau_{3}\right\}$. We thus number $\boldsymbol{R}_{\text {cls.2 }}^{l}$ serially (\#1)-(\# 3 ) and each result is displayed as follows:

(\#1) For the case of $\tau_{2}=\tau_{3}=0, \kappa_{2}+\imath \kappa_{3} \neq 0$,

$$
\begin{aligned}
\varpi & =\imath \eta_{3} \omega_{3} \\
\boldsymbol{\Omega}_{a}^{\imath}= & \imath e_{1 a}\left[\begin{array}{cc}
-\kappa_{2} & \tau_{1} \\
\imath \tau_{1} & \imath \kappa_{3}
\end{array}\right]+e_{2 a}\left[\begin{array}{cc}
0 & \eta_{2} \\
0 & \imath \eta_{3}
\end{array}\right]-\imath e_{3 a}\left[\begin{array}{cc}
-\eta_{2} & 0 \\
-\imath \eta_{3} & 0
\end{array}\right], \\
\boldsymbol{R}_{\text {cls.2 }}^{l \# 1}= & {\left[\begin{array}{ll}
£_{2} \kappa_{2} & £_{3} \kappa_{2} \\
£_{2} \kappa_{3} & £_{3} \kappa_{3} \\
£_{2} \eta_{2} & £_{3} \eta_{2} \\
£_{2} \eta_{3} & £_{3} \eta_{3} \\
£_{2} \tau_{1} & £_{3} \tau_{1}
\end{array}\right] . }
\end{aligned}
$$

(\#2) For the case of $\tau_{2}=\tau_{3} \neq 0$,

$$
\begin{aligned}
& \varpi=\left(\frac{£_{2} \kappa_{\delta}}{4 \tau_{2}}\right) \omega_{2}+\left(\frac{£_{3} \kappa_{\delta}}{4 \tau_{2}}+\imath \eta_{3}\right) \omega_{3}, \\
& \mathbf{\Omega}_{a}^{\imath}=\imath e_{1 a}\left[\begin{array}{cc}
-\frac{\kappa_{\delta}+\kappa_{\sigma}}{2} & \tau_{1}+\tau_{2} \\
\imath\left(\tau_{1}-\tau_{2}\right) & \frac{\kappa_{\delta}-\kappa_{\sigma}}{2}
\end{array}\right]+e_{2 a}\left[\begin{array}{cc}
0 & \eta_{2} \\
\frac{£_{2} \kappa_{\delta}}{4 \tau_{2}} & \frac{£_{3} \kappa_{\delta}}{4 \tau_{2}}+\imath \eta_{3}
\end{array}\right]-\imath e_{3 a}\left[\begin{array}{cc}
\frac{\imath £_{2} \kappa_{\delta}}{4 \tau_{2}}-\eta_{2} & \frac{\imath £_{3} \kappa_{\delta}}{4 \tau_{2}} \\
-\imath \eta_{3} & 0
\end{array}\right]
\end{aligned}
$$

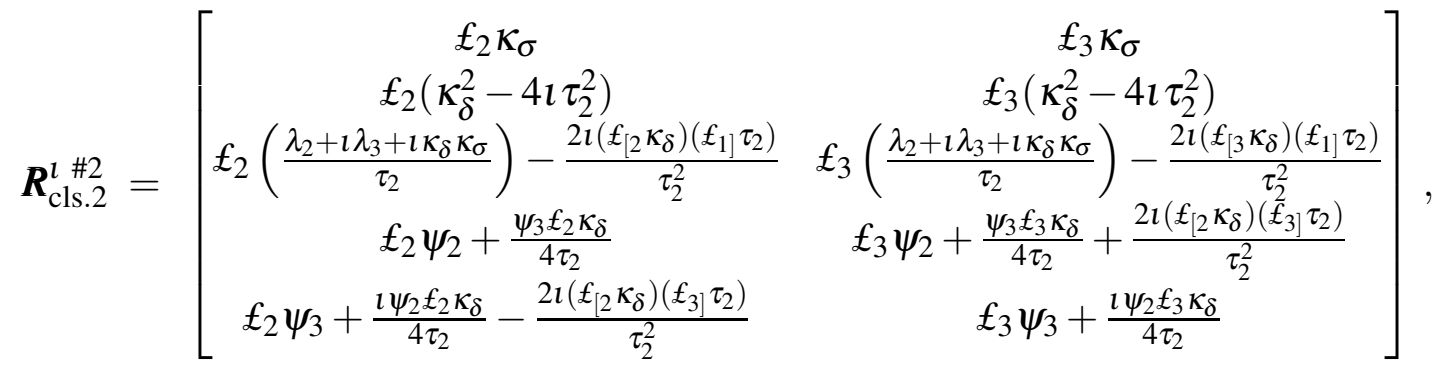

where

$$
\begin{array}{rlrl}
\lambda_{2} & \equiv R_{a b} e_{2}^{a} e_{2}^{b}, & \lambda_{3} \equiv R_{a b} e_{3}^{a} e_{3}^{b}, \\
\kappa_{\delta} \equiv \kappa_{2}+\imath \kappa_{3}, & \kappa_{\sigma} \equiv \kappa_{2}-\imath \kappa_{3}, \\
\psi_{\alpha} \equiv \frac{\imath £_{\alpha} \kappa_{\delta}}{\tau_{2}}+(-1)^{\alpha-1} 4 \eta_{\alpha} . & & (\alpha=2,3)
\end{array}
$$


(\#3) For the case of $\tau_{2} \neq \tau_{3}$,

$$
\begin{aligned}
& \varpi=-\left(\frac{£_{2} \kappa_{\sigma}+\imath £_{3} \tau_{\delta}-2 \imath \tau_{\delta} \eta_{2}}{2 \tau_{\delta}}\right) \omega_{2}-\left(\frac{£_{3} \kappa_{\sigma}+£_{2} \tau_{\delta}}{2 \tau_{\delta}}\right) \omega_{3}, \\
& \boldsymbol{\Omega}_{a}^{\iota}=\imath e_{1 a}\left[\begin{array}{cc}
-\frac{\kappa_{\delta}+\kappa_{\sigma}}{2} & \tau_{1}-\frac{\tau_{\delta}-\tau_{\sigma}}{2} \\
\imath \tau_{1}-\imath \frac{\tau_{\delta}+\tau_{\sigma}}{2} & \frac{\kappa_{\delta}-\kappa_{\sigma}^{2}}{2}
\end{array}\right]+e_{2 a}\left[\begin{array}{cc}
0 & \eta_{2} \\
\imath \eta_{2}-\frac{\varepsilon_{2} \kappa_{\sigma}+\imath £_{3} \tau_{\delta}}{2 \tau_{\delta}} & -\frac{£_{2} \tau_{\delta}+£_{3} \kappa_{\sigma}}{2 \tau_{\delta}}
\end{array}\right] \\
& -\imath e_{3 a}\left[\begin{array}{cc}
-\frac{\imath £_{2} \kappa_{\sigma}+£_{3} \tau_{\delta}}{2 \tau_{\delta}} & -\eta_{3}-\imath \frac{£_{3} \kappa_{\sigma}+£_{2} \tau_{\delta}}{2 \tau_{\delta}} \\
-\imath \eta_{3} & 0
\end{array}\right] \\
& \boldsymbol{R}_{\mathrm{cls.2}}^{l \# 3}=\left[\begin{array}{cc}
£_{2} \kappa_{\delta}-\imath £_{3} \tau_{\delta}+\left(\sigma_{2}-2 \imath \eta_{2}\right) \tau_{\sigma} & £_{3} \kappa_{\delta}+£_{2} \tau_{\delta}+\left(\sigma_{3}+2 \imath \eta_{3}\right) \tau_{\sigma} \\
£_{2}\left(\tau_{\sigma}+\tau_{\delta}\right)+\imath\left(\sigma_{2}-2 \imath \eta_{2}\right) \kappa_{\delta} & £_{3}\left(\tau_{\sigma}-\tau_{\delta}\right)+\imath\left(\sigma_{3}+2 \imath \eta_{3}\right) \kappa_{\delta} \\
£_{2} R_{11}-2 \imath \tau_{\delta} R_{13} & £_{3} R_{11}-2 \tau_{\delta} R_{12} \\
£_{2} \sigma_{2}-\frac{\sigma_{3}\left(\sigma_{2}-2 \imath \eta_{2}\right)}{2}-\left(\kappa_{\delta}+\kappa_{\sigma}\right) \tau_{\delta} & £_{2} \sigma_{3}-\frac{\sigma_{3}^{2}}{2}+\sigma_{2} \eta_{2}-\frac{\left(\tau_{\sigma}-\tau_{\delta}\right)^{2}}{2}-\Upsilon \\
£_{3} \sigma_{2}-\frac{\imath \sigma_{2}^{2}}{2}-\imath \sigma_{3} \eta_{3}+\frac{\left(\tau_{\sigma}+\tau_{\delta}\right)^{2}}{2}+\Upsilon & £_{3} \sigma_{3}-\frac{\imath \sigma_{2}\left(\sigma_{3}+2 \imath \eta_{3}\right)}{2}-\imath\left(\kappa_{\delta}-\kappa_{\sigma}\right) \tau_{\delta}
\end{array}\right],
\end{aligned}
$$

where $R_{i j} \equiv R_{a b} e_{i}^{a} e_{j}^{b}$ and

$$
\begin{array}{rlrl}
\tau_{\sigma} & \equiv \tau_{3}+\tau_{2}, & \tau_{\delta} \equiv \tau_{3}-\tau_{2}, \\
\sigma_{2} \equiv \frac{£_{2} \kappa_{\sigma}+\imath £_{3} \tau_{\delta}}{\tau_{\delta}}, & \sigma_{3} \equiv \frac{£_{3} \kappa_{\sigma}+£_{2} \tau_{\delta}}{\tau_{\delta}}, \\
\Upsilon & \equiv R_{22}-\imath\left(R_{11}+R_{33}\right)-\frac{\imath}{2}\left(\kappa_{\delta}^{2}-\kappa_{\sigma}^{2}\right) . &
\end{array}
$$

In a way parallel to that of $\boldsymbol{R}_{\mathrm{cls} .2}^{l}$, the rank of $\boldsymbol{R}_{\mathrm{cls} .2}^{l \# 1}, \boldsymbol{R}_{\mathrm{cls} .2}^{l \# 2}, \boldsymbol{R}_{\mathrm{cls} .2}^{l \# 3}$ controls the number of KVs. For instance, in the case of $\tau_{2}=\tau_{3}=0, \kappa_{2}+\imath \kappa_{3} \neq 0$, rank $\boldsymbol{R}_{\text {cls. } 2}^{l \# 1}=0$ implies that two $\mathrm{KVs}$ exist. If $\operatorname{rank} \boldsymbol{R}_{\mathrm{cls} .2}^{l \# 1}=2$, we have no KVs. Otherwise, $\operatorname{rank} \boldsymbol{R}_{\mathrm{cls} .2}^{l \# 1}=1$ and then the KVs in this branch are proportional to an annihilator of $\boldsymbol{R}_{a}$. As explained in 2.3, the sub-algorithm described in Figure 2a can be immediately testable.

\subsection{Null case}

In this case, we suppose that $\left\{u^{a}, v^{a}, e^{a}\right\}$ spans a double-null basis of $T(M)$,

$$
g^{a b}=u^{a} v^{b}+v^{a} u^{b}+e^{a} e^{b},
$$

where $\left\{u^{a}, v^{a}\right\}$ are null vectors such that $g_{a b} u^{a} v^{b}=1 ; e^{a}$ is a spacelike unit vector orthogonal to $u^{a}$ and $v^{a} ;\left\{u^{a}, e^{a}\right\}$ are two annihilators of $\boldsymbol{R}_{a}$.

When dim $\operatorname{ker} \boldsymbol{R}_{a}=2$ and the 1 -form in eq. (1.7b) is null, a null vector $u^{a}$ can be taken as its contravariant counterpart. By constructing another null vector $v^{a}$ satisfying $g_{a b} u^{a} v^{b}=1$, a spacelike unit vector $e^{a}$ can also taken as $e^{a} \equiv \varepsilon^{a b c} u_{b} v_{c}$.

Given the above assumptions, any KV can be written in the form

$$
K^{a}=\omega_{u} u^{a}+\omega_{e} e^{a},
$$


where $\left\{\omega_{u}, \omega_{e}\right\}$ are unknown scalars to be determined.

In order to write the components of eq. (1.1) with eq. (3.17) explicitly, we introduce the Ricci rotation coefficients as

$$
\begin{aligned}
u^{b} \nabla_{b}\left[\begin{array}{l}
u^{a} \\
v^{a} \\
e^{a}
\end{array}\right] & =\left[\begin{array}{ccc}
\kappa_{u} & 0 & \eta_{u} \\
0 & -\kappa_{u} & \tau_{u} \\
-\tau_{u} & -\eta_{u} & 0
\end{array}\right]\left[\begin{array}{l}
u^{a} \\
v^{a} \\
e^{a}
\end{array}\right], \\
v^{b} \nabla_{b}\left[\begin{array}{c}
u^{a} \\
v^{a} \\
e^{a}
\end{array}\right] & =\left[\begin{array}{ccc}
-\kappa_{v} & 0 & \tau_{v} \\
0 & \kappa_{v} & \eta_{v} \\
-\eta_{v} & -\tau_{v} & 0
\end{array}\right]\left[\begin{array}{c}
u^{a} \\
v^{a} \\
e^{a}
\end{array}\right], \\
e^{b} \nabla_{b}\left[\begin{array}{c}
u^{a} \\
v^{a} \\
e^{a}
\end{array}\right] & =\left[\begin{array}{ccc}
\tau_{e} & 0 & -\kappa_{e} \\
0 & -\tau_{e} & -\eta_{e} \\
\eta_{e} & \kappa_{e} & 0
\end{array}\right]\left[\begin{array}{c}
u^{a} \\
v^{a} \\
e^{a}
\end{array}\right] .
\end{aligned}
$$

where $\kappa_{u}, \eta_{u}$, and $\tau_{u}$ are respectively the geodesic, normal curvature and relative torsion of an integral curve of $u^{a}$. The same geometric interpretation is bestowed with quantities for $v^{a}$ and $e^{a}$. Their derivatives entail relations amongst each other, which are collected in Appendix A.2.

By using eqs. (3.17)-(3.18), the $u u$-component of eq. (1.1) reads

$$
\eta_{u} \omega_{e}=0 .
$$

Depending on whether $u^{a}$ satisfies the geodesic equation $u^{b} \nabla_{b} u^{a}=\kappa_{u} u^{a}$, the analysis branches off.

3.2.1. Branch where $u^{a}$ is not a geodesic tangent In this branch, $\eta_{u} \neq 0$. Hence eq. (3.19) implies $\omega_{e}=0$ and then eq. (3.17) takes the form

$$
K^{a}=\omega_{u} u^{a},
$$

which conforms with the assumption (2.1) of the class 1 . As explained in 2.3, the subalgorithm described in Figure 2a can be immediately testable.

3.2.2. Branch where $u^{a}$ is a geodesic tangent $\eta_{u}=0$ In this branch, the remaining parts of eq. (1.1) lead to

$$
\begin{aligned}
£_{u} \omega_{u} & =-\kappa_{u} \omega_{u}+\left(\tau_{u}+\tau_{v}\right) \omega_{e}, \\
£_{u} \omega_{e} & =-\kappa_{e} \omega_{e}, \\
£_{v} \omega_{u} & =\kappa_{v} \omega_{u}+\eta_{v} \omega_{e}, \\
£_{v} \omega_{e} & =\varpi, \\
£_{e} \omega_{u} & =-\left(\tau_{v}+\tau_{e}\right) \omega_{u}-\eta_{e} \omega_{e}-\varpi, \\
£_{e} \omega_{e} & =\kappa_{e} \omega_{u},
\end{aligned}
$$


where eq. (3.21d) defines the 1-jet variable $\varpi$. As is the case in Subsection 3.1, the PDE system (3.21) is not closed with respect to unknown scalars $\left\{\omega_{u}, \omega_{e}, \varpi\right\}$. The supplementary equations follow from several parts of the identities $\nabla_{[a} \nabla_{b]} \omega_{u}=\nabla_{[a} \nabla_{b]} \omega_{e}=0$, yielding

$$
\begin{aligned}
2 \tau_{v} \varpi= & \left(£_{u} \kappa_{v}+£_{v} \kappa_{u}+2 \kappa_{u} \kappa_{v}+\left(\tau_{u}-\tau_{v}\right)\left(\tau_{v}+\tau_{e}\right)\right) \omega_{u} \\
& -\left(£_{v}\left(\tau_{u}+\tau_{v}\right)-£_{u} \eta_{v}-\eta_{v}\left(2 \kappa_{u}-\kappa_{e}\right)-\eta_{e}\left(\tau_{u}-\tau_{v}\right)\right) \omega_{e}, \\
\kappa_{e} \varpi= & \left(£_{u} \tau_{v}+\kappa_{e}\left(\tau_{u}-\tau_{v}\right)\right) \omega_{u}+\left(£_{e} \tau_{v}-\kappa_{e} \eta_{e}\right) \omega_{e} .
\end{aligned}
$$

This implies that the 1-jet variable $\varpi$ is written in terms of $\omega_{u}$ and $\omega_{e}$ except for $\tau_{v}=\kappa_{e}=0$. Depending on the vanishing of the coefficients $\left\{\tau_{v}, \kappa_{e}\right\}$, the analysis falls into three subbranches.

Sub-branch where $\tau_{v}=\kappa_{e}=0 \quad$ In this sub-branch, the 1-jet variable $\varpi$ cannot be expressed in terms of $\omega_{u}$ and $\omega_{e}$. The differential equations for $\varpi$ come from the remaining parts of the identities $\nabla_{[a} \nabla_{b]} \omega_{u}=\nabla_{[a} \nabla_{b]} \omega_{e}=0$. By combining these with eqs. (3.21), we obtain a PDE system of the form

$$
\nabla_{a} \boldsymbol{\omega}=\boldsymbol{\Omega}_{a} \boldsymbol{\omega}, \quad \boldsymbol{\omega} \equiv\left[\begin{array}{lll}
\omega_{u} & \omega_{e} & \varpi
\end{array}\right]^{T},
$$

where

$$
\begin{aligned}
& \boldsymbol{\Omega}_{a} \equiv u_{a}\left[\begin{array}{ccc}
\kappa_{v} & \eta_{v} & 0 \\
0 & 0 & 1 \\
£_{v} \tau_{u}-£_{u} \eta_{v}-2 \eta_{v} \kappa_{u}-\eta_{e} \tau_{u} & -£_{v} \eta_{e}-£_{e} \eta_{v}+\eta_{e}\left(\eta_{e}+\kappa_{v}\right)+\eta_{v}\left(\tau_{u}-2 \tau_{e}\right) & \kappa_{v}
\end{array}\right] \\
& +v_{a}\left[\begin{array}{ccc}
-\kappa_{u} & \tau_{u} & 0 \\
0 & 0 & 0 \\
0 & 0 & -\kappa_{u}
\end{array}\right]+e_{a}\left[\begin{array}{ccc}
-\tau_{e} & -\eta_{e} & -1 \\
0 & 0 & 0 \\
0 & 0 & -\tau_{e}
\end{array}\right]
\end{aligned}
$$

The compatibility condition, $\left(\nabla_{[a} \boldsymbol{\Omega}_{b]}-\boldsymbol{\Omega}_{[a} \boldsymbol{\Omega}_{b]}\right) \boldsymbol{\omega}=0$, for eq. (3.23) reads

$$
\boldsymbol{R}_{\mathrm{cls} .2} \boldsymbol{\omega}=0, \quad \boldsymbol{R}_{\mathrm{cls} .2} \equiv\left[\begin{array}{ccc}
R_{u v} & R_{v e} & 0 \\
£_{e} R_{v e}+\tau_{e} R_{v e} & -£_{e} R_{v v}-2 \tau_{e} R_{v v}-\eta_{e} R_{v e} & -2 R_{v e}
\end{array}\right],
$$

where $R_{u v} \equiv R_{a b} u^{a} v^{b}, R_{v e} \equiv R_{a b} v^{a} e^{b}$ and $R_{v v} \equiv R_{a b} v^{a} v^{b}$. The first line corresponds to eq. (3.22a).

In this sub-branch, the rank of $\boldsymbol{R}_{\text {cls.2 }}$ influences the number of KVs in the same way as that presented in Subsection 3.1.2.

Other sub-branches Except for $\tau_{v}=\kappa_{e}=0$, eqs. (3.22) allow us to express the 1-jet variable $\varpi$ in terms of $\left\{\omega_{u}, \omega_{e}\right\}$. In these sub-branches, eq. (3.21) is closed with respect to $\left\{\omega_{u}, \omega_{e}\right\}$ and then the compatibility of PDE systems is of the form

$$
\nabla_{a} \boldsymbol{\omega}=\boldsymbol{\Omega}_{a} \boldsymbol{\omega}, \quad \boldsymbol{\omega} \equiv\left[\begin{array}{ll}
\omega_{u} & \omega_{e}
\end{array}\right]^{T} .
$$


The upshot is as follows:

(\#1) For the case of $\tau_{v}=0, \kappa_{e} \neq 0$,

$$
\begin{aligned}
\varpi= & \tau_{u} \omega_{u}-\eta_{e} \omega_{e}, \\
\boldsymbol{\Omega}_{a}= & u_{a}\left[\begin{array}{cc}
\kappa_{v} & \eta_{v} \\
\tau_{u} & -\eta_{e}
\end{array}\right]+v_{a}\left[\begin{array}{cc}
-\kappa_{u} & \tau_{u} \\
0 & -\kappa_{e}
\end{array}\right]+e_{a}\left[\begin{array}{cc}
-\tau_{u}-\tau_{e} & 0 \\
\kappa_{e} & 0
\end{array}\right], \\
\boldsymbol{R}_{\mathrm{cls} .2}^{\# 1}= & {\left[\begin{array}{cc}
R_{u v}-\frac{1}{2} R_{e e} & R_{v e}+\eta_{v} \kappa_{e} \\
£_{u} \tau_{u} & £_{e} \tau_{u} \\
£_{u} \kappa_{e}-\kappa_{u} \kappa_{e} & £_{e} \kappa_{e}-\tau_{e} \kappa_{e} \\
£_{u} \eta_{v}+2 \kappa_{u} \eta_{v} & £_{e} \eta_{v}+2 \tau_{e} \eta_{v} \\
£_{u} \eta_{e}+\kappa_{u} \eta_{e} & £_{e} \eta_{e}+\tau_{e} \eta_{e}
\end{array}\right], }
\end{aligned}
$$

where $R_{e e} \equiv R_{a b} e^{a} e^{b}$.

(\#2) For the case of $\tau_{v} \neq 0$,

$$
\begin{aligned}
& \varpi=\left(\frac{£_{u} \kappa_{v}+£_{v} \kappa_{u}+2 \kappa_{u} \kappa_{v}+\left(\tau_{u}-\tau_{v}\right)\left(\tau_{v}+\tau_{e}\right)}{2 \tau_{v}}\right) \omega_{u} \\
& -\left(\frac{£_{v}\left(\tau_{u}+\tau_{v}\right)-£_{u} \eta_{v}-\eta_{v}\left(2 \kappa_{u}-\kappa_{e}\right)-\eta_{e}\left(\tau_{u}-\tau_{v}\right)}{2 \tau_{v}}\right) \omega_{e},
\end{aligned}
$$

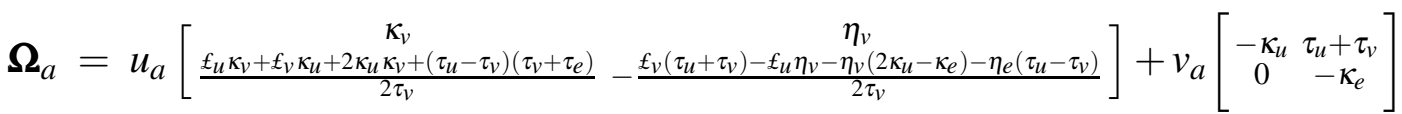

$$
\begin{aligned}
& +e_{a}\left[-\frac{f_{u} \kappa_{v}+\epsilon_{v} \kappa_{u}+2 \kappa_{u} \kappa_{v}+\left(\tau_{u}+\tau_{v}\right)\left(\tau_{v}+\tau_{e}\right)}{2 \tau_{v}} \frac{f_{v}\left(\tau_{u}+\tau_{v}\right)-\varepsilon_{u} \eta_{v}-\eta_{v}\left(2 \kappa_{u}-\kappa_{e}\right)-\eta_{e}\left(\tau_{u}+\tau_{v}\right)}{2 \tau_{v}}\right],
\end{aligned}
$$

and $\boldsymbol{R}_{\mathrm{cls} .2}^{\# 2} \boldsymbol{\omega}=0$ can be written as

$$
\begin{aligned}
0= & \left(£_{u} \kappa_{e}-\kappa_{u} \kappa_{e}\right) \omega_{u}+\left(£_{e} \kappa_{e}+\left(\tau_{v}-\tau_{e}\right) \kappa_{e}\right) \omega_{e} \\
0= & \left(2 £_{u} \tau_{v}+\left(\psi_{1}+2 \tau_{u}\right) \kappa_{e}\right) \omega_{u}+\left(2 £_{e} \tau_{v}+\left(\psi_{2}-2 \eta_{e}\right) \kappa_{e}\right) \omega_{e} \\
0= & \left(£_{u} \psi_{1}\right) \omega_{u}+\left(£_{u} \psi_{2}+\left(\kappa_{u}-\kappa_{e}\right) \psi_{2}+\left(\tau_{u}+\tau_{v}\right) \psi_{1}+2 \tau_{u} \tau_{v}-R_{e e}\right) \omega_{e} \\
0= & \left(£_{v}\left(\psi_{1}-2 \tau_{v}\right)-\frac{\psi_{2}}{2}\left(\psi_{1}-2 \tau_{v}\right)-2 \eta_{v} \kappa_{e}\right) \omega_{u} \\
& +\left(£_{v} \psi_{2}-\frac{\psi_{2}}{2}\left(\psi_{2}+2 \kappa_{v}\right)+\left(\psi_{1}+2 \tau_{v}\right) \eta_{v}-2 R_{v v}\right) \omega_{e} \\
0= & \left(£_{e} \psi_{1}+\frac{\psi_{1}^{2}}{2}+\kappa_{e} \psi_{2}+R_{e e}\right) \omega_{u}+\left(£_{e} \psi_{2}+\frac{\psi_{1}}{2}\left(\psi_{2}-2 \eta_{e}\right)+\tau_{e} \psi_{2}-2 \eta_{e} \tau_{v}\right) \omega_{e},
\end{aligned}
$$

where

$$
\psi_{1} \equiv \frac{1}{\tau_{v}}\left(R_{u v}-\frac{1}{2} R_{e e}\right)-\tau_{v}, \quad \psi_{2} \equiv \frac{1}{\tau_{v}}\left(R_{v e}+\eta_{v} \kappa_{e}+£_{v} \tau_{v}\right)
$$

In these sub-branches, the rank of $\boldsymbol{R}_{\text {cls.2 }}^{\# 1}$ and $\boldsymbol{R}_{\text {cls. } 2}^{\# 2}$ governs the number of KVs in the same way as that presented in Subsection 3.1.2. 


\subsection{Short summary of class 2}

We summarise the results obtained in this section in Figures $3 a-3 c$.

\section{class 2}

Either of two annihilators of $\boldsymbol{R}_{a}$ is null $\stackrel{\text { yes }}{\longrightarrow}$ class 2 null no

Figure 3a. The sub-algorithm for the class 2. For details, see the beginning of this section.

\section{class 2 non-null}

- The third vector is a geodesic tangent $\longrightarrow$ no

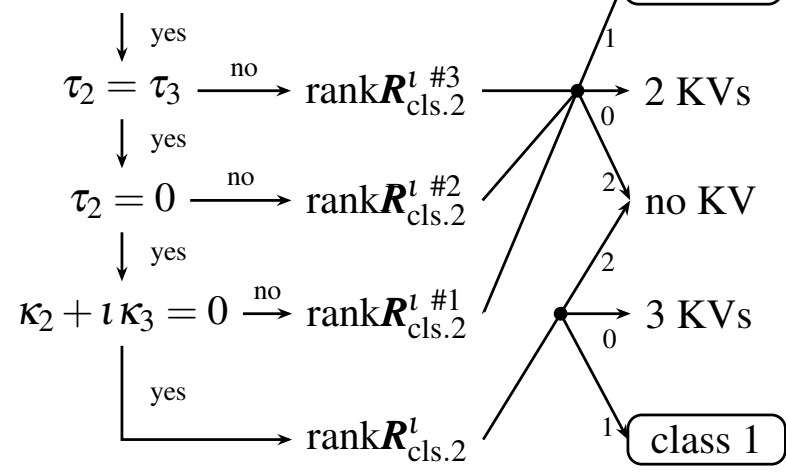

Figure 3b. The sub-algorithm for the class 2 non-null. See eqs. (3.5), (3.12)-(3.15) for notations.

class 2 null

- The null annihilator is a geodesic tangent $\stackrel{\text { no }}{\longrightarrow}$ class 1

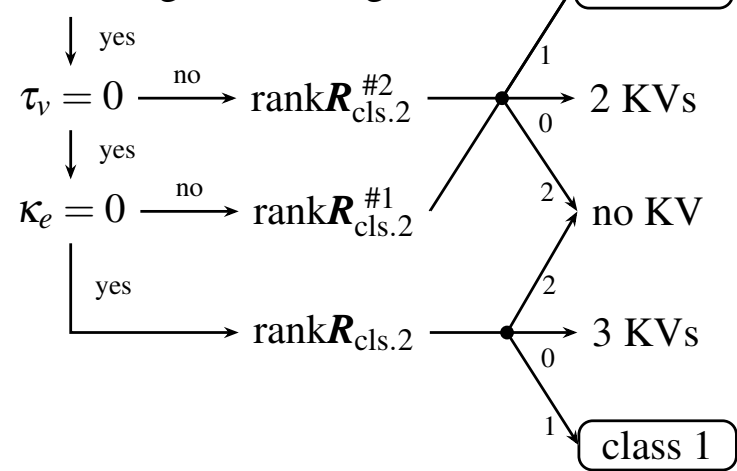

Figure 3c. The sub-algorithm for the class 2 null. See eqs. (3.18), (3.25)-(3.27) for notations. 
It should be emphasised that the sub-algorithm in Figures $3 a-3 c$ can be again applicatory for some cases in class 3 as explained in Section 1: At the outset, KVs in class 3 is expressed as a linear combination of the three annihilators of $\boldsymbol{R}_{a}$. In some branches, the KVs eventuates into the form of a linear combination of two (or less) annihilators of $\boldsymbol{R}_{a}$, while keeping the property $\operatorname{dim} \operatorname{ker} \boldsymbol{R}_{a}=3$. Since $K^{a}=\sum_{\alpha}^{2} \omega_{\alpha} u_{\alpha}^{a}$ accords with the prerequisite of class 2 (3.1), the results in this section is adapted to such branches as well.

\section{Analysis of class 3}

In this section, we address the case of class 3, for which all criteria in eqs. (1.7) are vanishing. This implies $\boldsymbol{R}_{a}$ is a zero matrix, whence any vector can be an annihilator of $\boldsymbol{R}_{a}$. Since the first obstruction matrix $\boldsymbol{R}_{a}$ has been intentionally designed to ensure that all the eigenvalues of the traceless Ricci operator $S^{a}{ }_{b}$ are constants if $\operatorname{rank} \boldsymbol{R}_{a}$ is zero, it is thereby reasonable to resort to the Jordan basis of $S^{a}{ }_{b}$. Perhaps the other choices for the basis of $T(M)$ fail to lessen the burden of computations, despite the fact that Jordan basis inevitably demands us to solve the eigenvalue problem. Thus, our proposed formulation is based on the Jordan decomposition of the matrix $S^{a}{ }_{b}$, which is nothing but the classification of the Segre type of $S^{a}{ }_{b}$. It can be found in [16] that the Segre classification is carried out by an examination of the minimal polynomial of $S^{a}{ }_{b}$ as shown in Figure 4a. See also [17] for the Segre classification of symmetric tensors in Lorentzian geometry.

Let us pause here to declare the Segre notation [11]. The eigenvalue equation $S^{a}{ }_{b} V^{b}=$ $\lambda V^{a}$ determines the orders of elementary divisors which belong to the several eigenvalues. A characteristic feature in Lorentzian geometry is that the elementary divisors can be non-simple and the eigenvalues can be complex. The Segre notation stands for the orders of elementary divisors with the round brackets specifying which eigenvalues coincide. If two eigenvalues are complex conjugates, they are denoted by $z$ and $\bar{z}$.

With these notations in mind, we are proceeding along four cases: We discuss the Segre type $[1,11]$ in Subsection 4.1, the Segre type [21] in Subsection 4.2, the Segre type [3] in Subsection 4.3 and the Segre type $[z \bar{z} 1]$ in Subsection 4.4 .

\subsection{Type $[1,11]$ and its degeneracies}

In this case, we have the following Jordan chains:

$$
S^{a}{ }_{b} e_{\alpha}^{b}=\lambda_{\alpha} e_{\alpha}^{a}, \quad(\alpha=1,2,3)
$$

with $\sum_{\alpha} \lambda_{\alpha}=0$. Here $e_{\alpha}^{a}$ is an eigenvector of $S^{a}{ }_{b}$ belong to the eigenvalue $\lambda_{\alpha}$. In this subsection, it is assumed that $\left\{e_{\alpha}^{a}\right\}$ are normalised and $e_{1}^{a}$ is timelike, so that $\left\{e_{\alpha}^{a}\right\}$ form an orthonormal basis of $T(M)$,

$$
g^{a b}=-e_{1}^{a} e_{1}^{b}+e_{2}^{a} e_{2}^{b}+e_{3}^{a} e_{3}^{b} .
$$




\section{class 3}

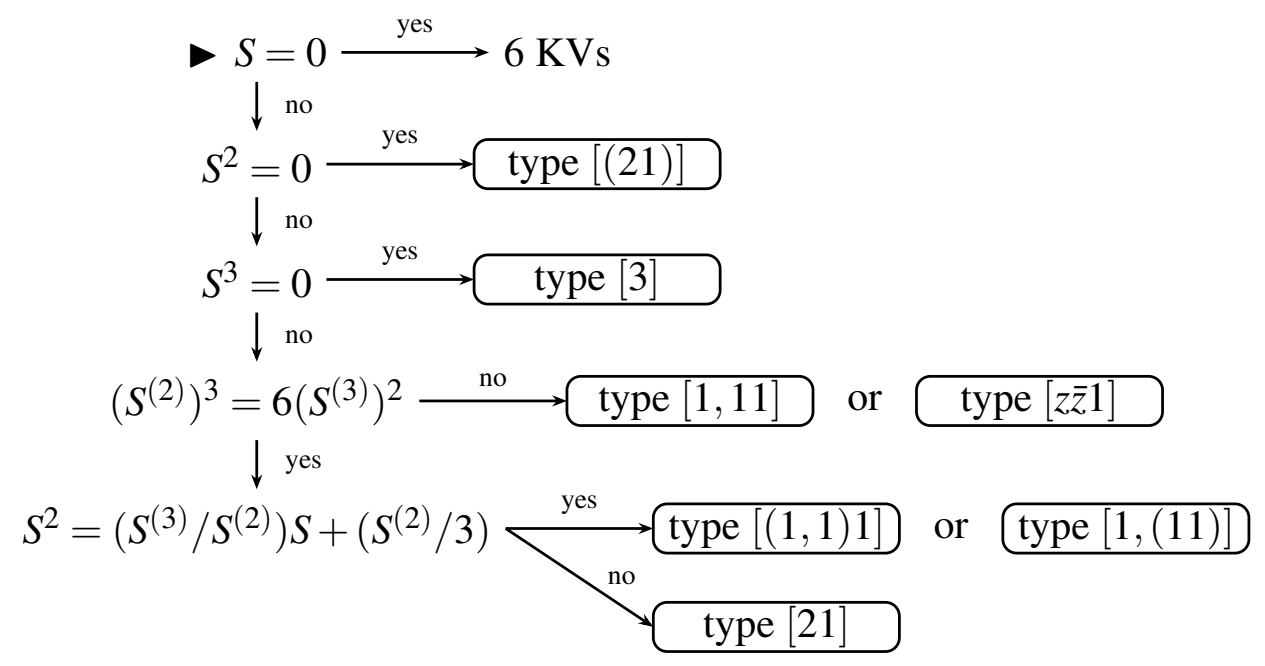

Figure 4a. The sub-algorithm for the class 3 . The notation is as follows: The two indices on the traceless Ricci operator $S^{a}{ }_{b}$ are dropped for short, e.g. $S^{2}$ denotes $S_{b}^{a} S^{b}{ }_{c} ; S^{(i)}$ denotes the $g$-trace of $S^{i}(i=2,3)$.

Then, any KV can be written as

$$
K^{a}=\sum_{\alpha=1}^{3} \omega_{\alpha} e_{\alpha}^{a},
$$

where scalars $\left\{\omega_{\alpha}\right\}$ are yet indeterminate.

It is an elementary computation to write down the the first compatibility condition, $£_{K} S_{a b}=0$, of eq. (1.1), giving

$$
\begin{aligned}
& 0=\left(\lambda_{1}-\lambda_{2}\right)\left(\varpi_{2}+\kappa_{2} \omega_{2}-\left(\tau_{1}+\tau_{3}\right) \omega_{3}\right), \\
& 0=\left(\lambda_{2}-\lambda_{3}\right)\left(\varpi_{3}-\left(\tau_{1}-\tau_{2}\right) \omega_{1}+\eta_{3} \omega_{3}\right), \\
& 0=\left(\lambda_{3}-\lambda_{1}\right)\left(\varpi_{1}-\eta_{1} \omega_{1}-\left(\tau_{2}-\tau_{3}\right) \omega_{3}\right),
\end{aligned}
$$

where the Ricci rotation coefficients are defined by eqs. (3.5), and the 1-jet variables $\left\{\varpi_{\alpha}\right\}$ are respectively defined as

$$
\varpi_{1} \equiv £_{3} \omega_{1}, \quad \varpi_{2} \equiv £_{1} \omega_{2}, \quad \varpi_{3} \equiv £_{2} \omega_{3} .
$$

The eigenvalues $\lambda_{\alpha}$ are constrained by the second Bianchi identity $\nabla_{a} R_{b}^{a}-(1 / 2) \nabla_{b} R=0$ as

$$
\begin{aligned}
& 0=\left(\lambda_{1}-\lambda_{2}\right) \kappa_{2}-\left(\lambda_{3}-\lambda_{1}\right) \kappa_{3}, \\
& 0=\left(\lambda_{1}-\lambda_{2}\right) \kappa_{1}+\left(\lambda_{2}-\lambda_{3}\right) \eta_{3}, \\
& 0=\left(\lambda_{2}-\lambda_{3}\right) \eta_{2}+\left(\lambda_{3}-\lambda_{1}\right) \eta_{1} .
\end{aligned}
$$

The compatibility conditions (4.4) are fulfilled trivially if the Segre type is $[(1,11)]$, $\lambda_{1}=\lambda_{2}=\lambda_{3}=0$, thereby yielding the result shown in Figure 4a. In the remaining parts of this subsection, we investigate the Segre types $[1,(11)],[(1,1) 1]$ and $[1,11]$ separately. 
4.1.1. Branch where the Segre type is $[1,(11)]$ In this branch, two eigenvalues in the spacelike direction coincide, i.e. $\lambda_{2}=\lambda_{3}=-(1 / 2) \lambda_{1}$. Then, it immediately follows from eqs. (4.4) and (4.6) that

$$
\begin{array}{lll}
\kappa_{1}=0, & \eta_{1}=0, & \kappa_{3}=-\kappa_{2}, \\
\varpi_{1}=\left(\tau_{2}-\tau_{3}\right) \omega_{2}, & \varpi_{2}=-\kappa_{2} \omega_{2}+\left(\tau_{1}+\tau_{3}\right) \omega_{2} . &
\end{array}
$$

Given these conditions (4.7), the Killing equation (1.1) and the identities $\nabla_{[a} \nabla_{b]} \omega_{1}=$ $\nabla_{[a} \nabla_{b]} \omega_{2}=\nabla_{[a} \nabla_{b]} \omega_{3}=0$ produce a PDE system of the form

$$
\nabla_{a} \boldsymbol{\omega}=\boldsymbol{\Omega}_{a} \boldsymbol{\omega}, \quad \boldsymbol{\omega} \equiv\left[\begin{array}{llll}
\omega_{1} & \omega_{2} & \omega_{3} & \varpi_{3}
\end{array}\right]^{T},
$$

where

$$
\begin{aligned}
& \boldsymbol{\Omega}_{a} \equiv-e_{1 a}\left[\begin{array}{cccc}
0 & 0 & 0 & 0 \\
0 & -\kappa_{2} & \tau_{1}+\tau_{3} & 0 \\
0 & -\tau_{1}+\tau_{2} & \kappa_{2} & 0 \\
-2 \kappa_{2} \tau_{1} & \kappa_{2} \eta_{2}-£_{1} \eta_{2} & -\left(\tau_{1}+\tau_{3}\right) & \eta_{2}
\end{array}\right] \\
& +e_{2 a}\left[\begin{array}{cccc}
0 & 0 & -\tau_{2}+\tau_{3} & 0 \\
\kappa_{2} & 0 & \eta_{2} & 0 \\
0 & 0 & 0 & 1 \\
-\kappa_{2} \eta_{2}-£_{2} \tau_{2} & -£_{2} \eta_{2} & \eta_{3}^{2}-\left(\tau_{1}-\tau_{2}\right)\left(\tau_{2}-\tau_{3}\right)-£_{2} \eta_{3}-£_{3} \eta_{2} & 0
\end{array}\right] \\
& +e_{3 a}\left[\begin{array}{cccc}
0 & \tau_{2}-\tau_{3} & 0 & 0 \\
-\tau_{2}-\tau_{3} & -\eta_{2} & -\eta_{3} & -1 \\
-\kappa_{2} & \eta_{3} & 0 & 0 \\
2 \kappa_{2} \eta_{3}-£_{2} \kappa_{2} & -\eta_{3}^{2}+\left(\tau_{1}-\tau_{2}\right)\left(\tau_{2}-\tau_{3}\right)+£_{2} \eta_{3} & \eta_{2} \eta_{3} & \eta_{2}
\end{array}\right] \text {. }
\end{aligned}
$$

The compatibility condition for eq. (4.8) leads to

$$
\begin{aligned}
\left(\tau_{2}+\tau_{3}\right) \varpi_{3} & =-\tau_{2}\left(\tau_{2}+\tau_{3}\right) \omega_{1}+\left(£_{3} \tau_{2}-\eta_{2}\left(\tau_{2}+\tau_{3}\right)+2 \eta_{3} \kappa_{2}\right) \omega_{2}-\left(£_{2} \tau_{3}-2 \eta_{2} \kappa_{2}\right) \omega_{3} \\
4 \kappa_{2} \varpi_{3} & =-4 \kappa_{2} \tau_{2} \omega_{1}-\left(£_{2}\left(\tau_{2}+\tau_{3}\right)\right) \omega_{2}-\left(£_{3}\left(\tau_{2}+\tau_{3}\right)+4 \eta_{3} \kappa_{2}\right) \omega_{3} .
\end{aligned}
$$

This implies that $\varpi_{3}$ can be expressed in terms of $\left\{\omega_{1}, \omega_{2}, \omega_{3}\right\}$ except for $\kappa_{2}=\tau_{2}+\tau_{3}=0$. Depending on the nonzeroness of the coefficients $\left\{\kappa_{2}, \tau_{2}+\tau_{3}\right\}$, the analysis falls into three sub-branches.

Sub-branch where $\kappa_{2}=\tau_{2}+\tau_{3}=0 \quad$ In this sub-branch, the conditions (4.10) gives

$$
\left(£_{2} \tau_{2}\right) \omega_{2}+\left(£_{3} \tau_{2}\right) \omega_{3}=0
$$

whilst the relations in eqs. (A.3) imply that $£_{2} \tau_{2}=£_{3} \tau_{2}=0$. We conclude that four KVs exist in this sub-branch, since the compatibility is trivially fulfilled.

Other sub-branches Except for $\kappa_{2}=\tau_{2}+\tau_{3}=0$, the 1-jet variable $\bar{\varpi}_{3}$ is not an independent variable, but is expressed in in terms of $\left\{\omega_{1}, \omega_{2}, \omega_{3}\right\}$ by virtue of eqs. (4.10). In these subbranches, eq. (4.8) is closed with respect to $\left\{\omega_{1}, \omega_{2}, \omega_{3}\right\}$ and then the compatibility of PDE systems is of the form

$$
\nabla_{a} \boldsymbol{\omega}=\boldsymbol{\Omega}_{a} \boldsymbol{\omega}, \quad \boldsymbol{\omega} \equiv\left[\begin{array}{lll}
\omega_{1} & \omega_{2} & \omega_{3}
\end{array}\right]^{T} .
$$


The results for two cases (\#1) and (\#2) are presented as follows:

(\#1) For the case of $\tau_{2}+\tau_{3}=0, \kappa_{2} \neq 0$,

$$
\begin{aligned}
\varpi_{3} & =-\tau_{2} \omega_{1}-\eta_{3} \omega_{3} \\
\boldsymbol{\Omega}_{a} & =-e_{1 a}\left[\begin{array}{ccc}
0 & 0 & 0 \\
0 & -\kappa_{2} & -\tau_{2} \\
0 & \tau_{2} & \kappa_{2}
\end{array}\right]+e_{2 a}\left[\begin{array}{ccc}
0 & 0 & -2 \tau_{2} \\
\kappa_{2} & 0 & \eta_{2} \\
-\tau_{2} & 0 & -\eta_{3}
\end{array}\right]+e_{3 a}\left[\begin{array}{ccc}
0 & 2 \tau_{2} & 0 \\
\tau_{2} & -\eta_{2} & 0 \\
-\kappa_{2} & \eta_{3} & 0
\end{array}\right], \\
\boldsymbol{R}_{[1,(11)]}^{\# 11} & =\left[\begin{array}{ccc}
0 & £_{2} \tau_{2} & £_{3} \tau_{2} \\
£_{1} \eta_{2} & £_{2} \eta_{2} & £_{3} \eta_{2} \\
£_{1} \eta_{3} & £_{2} \eta_{3} & £_{3} \eta_{3}
\end{array}\right]
\end{aligned}
$$

(\#2) For the case of $\tau_{2}+\tau_{3} \neq 0$,

$$
\begin{aligned}
& \varpi_{3}=\left(\frac{\tau_{\delta}-\tau_{\sigma}}{2}\right) \omega_{1}+\left(\frac{£_{2} \kappa_{2}}{\tau_{\sigma}}\right) \omega_{2}+\left(\frac{£_{3} \kappa_{2}}{\tau_{\sigma}}-\eta_{3}\right) \omega_{3}, \\
& \boldsymbol{\Omega}_{a}=-e_{1 a}\left[\begin{array}{ccc}
0 & 0 & 0 \\
0 & -\kappa_{2} & \tau_{1}+\frac{\tau_{\delta}+\tau_{\sigma}}{2} \\
0 & -\tau_{1}-\frac{\tau_{\delta}-\tau_{\sigma}}{2} & \kappa_{2}
\end{array}\right]+e_{2 a}\left[\begin{array}{ccc}
0 & 0 & \tau_{\delta} \\
\kappa_{2} & 0 & \eta_{2} \\
\frac{\tau_{\delta}-\tau_{\sigma}}{2} & \frac{f_{2} \kappa_{2}}{\tau_{\sigma}} & \frac{f_{3} \kappa_{2}}{\tau_{\sigma}}-\eta_{3}
\end{array}\right] \\
& +e_{3 a}\left[\begin{array}{ccc}
0 & -\tau_{\delta} & 0 \\
-\frac{\tau_{\delta}+\tau_{\sigma}}{2} & -\frac{£_{2} \kappa_{2}}{\tau_{\sigma}}-\eta_{2} & -\frac{f_{3} \kappa_{2}}{\tau_{\sigma}} \\
-\kappa_{2} & \eta_{3} & 0
\end{array}\right], \\
& \boldsymbol{R}_{[1,(11)]}^{\# 2}=\left[\begin{array}{ccc}
0 & £_{2} \tau_{\delta} & £_{3} \tau_{\delta} \\
\Pi_{1} & \Pi_{2} & \Pi_{3} \\
\Xi_{1} & \Xi_{2} & \Xi_{3}
\end{array}\right],
\end{aligned}
$$

where

$$
\begin{aligned}
\tau_{\delta} & \equiv \tau_{3}-\tau_{2} \\
\tau_{\sigma} & \equiv \tau_{3}+\tau_{2} \\
\Pi_{\alpha} & \equiv £_{\alpha} \eta_{2}+\frac{£_{\alpha} £_{2} \kappa_{2}}{\tau_{\sigma}}-\left(£_{2}\left(\tau_{\delta}+3 \tau_{\sigma}\right)-4 \eta_{2} \kappa_{2}\right) \frac{£_{\alpha} \kappa_{2}}{2 \tau_{\sigma}^{2}} \\
\Xi_{\alpha} & \equiv £_{\alpha} \eta_{3}-\frac{£_{\alpha} £_{3} \kappa_{2}}{\tau_{\sigma}}-\left(£_{3}\left(\tau_{\delta}-3 \tau_{\sigma}\right)-4 \eta_{3} \kappa_{2}\right) \frac{£_{\alpha} \kappa_{2}}{2 \tau_{\sigma}^{2}}
\end{aligned}
$$

In these sub-branches, the rank of $\boldsymbol{R}_{[1,(11)]}^{\# 1}$ and $\boldsymbol{R}_{[1,(11)]}^{\# 2}$ is linked to the number of KVs as follows: If $\operatorname{rank} \boldsymbol{R}_{[1,(11)]}^{\# 1}=0$, three $\mathrm{KVs}$ exist; If $\operatorname{rank} \boldsymbol{R}_{[1,(11)]}^{\# 1}=3$, there is no $\mathrm{KV}$; If $\operatorname{rank} \boldsymbol{R}_{[1,(11)]}^{\# 1}=2$, the sub-algorithm described in Figure 2a can be testable since the KVs in this case are proportional to an annihilator of $\boldsymbol{R}_{a}$; Otherwise $\operatorname{rank} \boldsymbol{R}_{[1,(11)]}^{\# 1}=1$, the sub-algorithm described in Figures 3a-3c can be testable since the KVs in this case can be written as a linear combination of two annihilators of $\boldsymbol{R}_{a}$. The argument for $\boldsymbol{R}_{[1,(11)]}^{\# 2}$ parallels with above. 
4.1.2. Branch where the Segre type is $[(1,1) 1] \quad$ In this branch, we have $\lambda_{1}=\lambda_{3}=-(1 / 2) \lambda_{2}$. Then, it immediately follows from eqs. (4.4) and (4.6) that

$$
\begin{array}{lll}
\kappa_{2}=0, & \eta_{2}=0, & \kappa_{1}=\eta_{3} \\
\varpi_{2}=\left(\tau_{1}+\tau_{3}\right) \omega_{3}, & \varpi_{3}=\left(\tau_{1}-\tau_{2}\right) \omega_{1}-\eta_{3} \omega_{3} . &
\end{array}
$$

Given these conditions (4.14), the Killing equation (1.1) and the identities $\nabla_{[a} \nabla_{b]} \omega_{1}=$ $\nabla_{[a} \nabla_{b]} \omega_{2}=\nabla_{[a} \nabla_{b]} \omega_{3}=0$ produce a PDE system of the form

$$
\nabla_{a} \boldsymbol{\omega}=\boldsymbol{\Omega}_{a} \boldsymbol{\omega}, \quad \boldsymbol{\omega} \equiv\left[\begin{array}{llll}
\omega_{1} & \omega_{2} & \omega_{3} & \varpi_{1}
\end{array}\right]^{T},
$$

where

$$
\begin{aligned}
\boldsymbol{\Omega}_{a} \equiv & -e_{1 a}\left[\begin{array}{cccc}
0 & -\kappa_{1} & -\eta_{1} & 0 \\
0 & 0 & \tau_{1}+\tau_{3} & 0 \\
-\eta_{1} & -\tau_{1}+\tau_{3} & -\kappa_{3} & 1 \\
-\eta_{1} \kappa_{3}-£_{3} \kappa_{1}-2 \eta_{1} \kappa_{1}-\eta_{1}^{2}+\left(\tau_{1}+\tau_{3}\right)\left(\tau_{2}-\tau_{3}\right)-£_{3} \eta_{1} & \kappa_{3}
\end{array}\right] \\
& +e_{2 a}\left[\begin{array}{cccc}
\kappa_{1} & 0 & -\tau_{2}+\tau_{3} & 0 \\
0 & 0 & 0 & 0 \\
\tau_{1}-\tau_{2} & 0 & -\kappa_{1} & 0 \\
\left(\tau_{1}-\tau_{2}\right) \kappa_{3} & -2 \kappa_{1} \tau_{2} & f_{2} \kappa_{3}-\kappa_{1} \kappa_{3} & 0
\end{array}\right] \\
& +e_{3 a}\left[\begin{array}{cccc}
0 & 0 & 0 & 1 \\
-\tau_{1}-\tau_{3} & 0 & 0 & 0 \\
\kappa_{3} & \kappa_{1} & 0 & 0 \\
\eta_{1}^{2}-\left(\tau_{1}+\tau_{3}\right)\left(\tau_{2}-\tau_{3}\right)+£_{1} \kappa_{3}+£_{3} \eta_{1} & \kappa_{1} \kappa_{3}-£_{3} \tau_{3} £_{3} \kappa_{3} & 0
\end{array}\right] .
\end{aligned}
$$

The compatibility condition for eq. (4.15) leads to

$$
\begin{gathered}
\left(\tau_{1}-\tau_{3}\right) \varpi_{1}=\left(2 \kappa_{1} \kappa_{3}-£_{3} \tau_{1}\right) \omega_{1}-\tau_{3}\left(\tau_{1}-\tau_{3}\right) \omega_{2}-\left(2 \eta_{1} \kappa_{1}-\kappa_{3}\left(\tau_{1}-\tau_{3}\right)-£_{1} \tau_{3}\right) \omega_{3} \\
4 \kappa_{1} \varpi_{1}=\left(4 \eta_{1} \kappa_{1}+£_{1}\left(\tau_{1}-\tau_{3}\right)\right) \omega_{1}-4 \kappa_{1} \tau_{3} \omega_{2}+\left(£_{3}\left(\tau_{1}-\tau_{3}\right)\right) \omega_{3}
\end{gathered}
$$

This implies that $\varpi_{1}$ can be expressed in terms of $\left\{\omega_{1}, \omega_{2}, \omega_{3}\right\}$ except for $\kappa_{1}=\tau_{1}-\tau_{3}=0$. Depending on whether the coefficients $\left\{\kappa_{1}, \tau_{1}-\tau_{3}\right\}$ of $\varpi$ are vanishing, the analysis falls into three sub-branches.

$\underline{\text { Sub-branch where } \kappa_{1}=\tau_{1}-\tau_{3}=0} \quad$ In this sub-branch, the conditions (4.17) gives

$$
\left(£_{1} \tau_{1}\right) \omega_{1}+\left(£_{3} \tau_{1}\right) \omega_{3}=0
$$

whilst the relations in eqs. (A.3) imply that $£_{1} \tau_{1}=£_{3} \tau_{1}=0$. We conclude that four KVs exist in this sub-branch, as the compatibility conditions are trivially met.

Other sub-branches Except when $\kappa_{1}=\tau_{1}-\tau_{3}=0$, eqs. (4.17) allow us to write the 1-jet variable $\Phi_{1}$ in terms of $\left\{\omega_{1}, \omega_{2}, \omega_{3}\right\}$. In these sub-branches, eq. (4.15) is closed with respect to $\left\{\omega_{1}, \omega_{2}, \omega_{3}\right\}$ and then the compatibility of PDE systems takes the form

$$
\nabla_{a} \boldsymbol{\omega}=\boldsymbol{\Omega}_{a} \boldsymbol{\omega}, \quad \boldsymbol{\omega} \equiv\left[\begin{array}{lll}
\omega_{1} & \omega_{2} & \omega_{3}
\end{array}\right]^{T} .
$$


The individual results are described as follows:

(\#1) For the case of $\tau_{1}=\tau_{3}, \kappa_{1} \neq 0$,

$$
\begin{aligned}
\varpi_{1} & =\eta_{1} \omega_{1}-\tau_{1} \omega_{2} \\
\boldsymbol{\Omega}_{a} & =-e_{1 a}\left[\begin{array}{ccc}
0 & -\kappa_{1} & -\eta_{1} \\
0 & 0 & 2 \tau_{1} \\
0 & -\tau_{1} & -\kappa_{3}
\end{array}\right]+e_{2 a}\left[\begin{array}{ccc}
\kappa_{1} & 0 & \tau_{1} \\
0 & 0 & 0 \\
\tau_{1} & 0 & -\kappa_{1}
\end{array}\right]+e_{3 a}\left[\begin{array}{ccc}
\eta_{1} & -\tau_{1} & 0 \\
-2 \tau_{1} & 0 & 0 \\
\kappa_{3} & \kappa_{1} & 0
\end{array}\right], \\
\boldsymbol{R}_{[(1,1) 1]}^{\# 1} & =\left[\begin{array}{lll}
£_{1} \tau_{1} & 0 & £_{3} \tau_{1} \\
£_{1} \eta_{1} & £_{2} \eta_{1} & £_{3} \eta_{1} \\
£_{1} \kappa_{3} & £_{2} \kappa_{3} & £_{3} \kappa_{3}
\end{array}\right],
\end{aligned}
$$

(\#2) For the case of $\tau_{1} \neq \tau_{3}$,

$$
\begin{aligned}
& \varpi_{1}=\left(\eta_{1}-\frac{£_{1} \kappa_{1}}{\tau_{\delta}}\right) \omega_{1}-\left(\frac{\tau_{\delta}+\tau_{\sigma}}{2}\right) \omega_{2}-\left(\frac{£_{3} \kappa_{1}}{\tau_{\delta}}\right) \omega_{3}, \\
& \boldsymbol{\Omega}_{a}=-e_{1 a}\left[\begin{array}{ccc}
0 & -\kappa_{1} & -\eta_{1} \\
0 & 0 & \tau_{\sigma} \\
-\frac{\varepsilon_{1} \kappa_{1}}{\tau_{\delta}} & \frac{\tau_{\delta}-\tau_{\sigma}}{2} & -\frac{\varepsilon_{3} \kappa_{1}}{\tau_{\delta}}-\kappa_{3}
\end{array}\right]+e_{2 a}\left[\begin{array}{ccc}
\kappa_{1} & 0 & \frac{\tau_{\delta}+\tau_{\sigma}}{2}-\tau_{2} \\
0 & 0 & 0 \\
-\frac{\tau_{\delta}-\tau_{\sigma}}{2}-\tau_{2} & 0 & -\kappa_{1}
\end{array}\right] \\
& +e_{3 a}\left[\begin{array}{ccc}
\eta_{1}-\frac{f_{1} \kappa_{1}}{\tau_{\delta}} & -\frac{\tau_{\delta}+\tau_{\sigma}}{2} & -\frac{f_{3} \kappa_{1}}{\tau_{\delta}} \\
-\tau_{\sigma} & 0 & 0 \\
\kappa_{3} & \kappa_{1} & 0
\end{array}\right] \\
& \boldsymbol{R}_{[(1,1) 1]}^{\# 2}=\left[\begin{array}{ccc}
£_{1} \tau_{\sigma} & 0 & £_{3} \tau_{\sigma} \\
\Pi_{1} & \Pi_{2} & \Pi_{3} \\
\Xi_{1} & \Xi_{2} & \Xi_{3}
\end{array}\right]
\end{aligned}
$$

where

$$
\begin{aligned}
\tau_{\sigma} & \equiv \tau_{3}+\tau_{1} \\
\tau_{\delta} & \equiv \tau_{3}-\tau_{1} \\
\Pi_{\alpha} & \equiv £_{\alpha} \eta_{1}-\frac{£_{\alpha} £_{1} \kappa_{1}}{\tau_{\delta}}+\left(£_{1}\left(\tau_{\sigma}+3 \tau_{\delta}\right)-4 \eta_{1} \kappa_{1}\right) \frac{£_{\alpha} \kappa_{1}}{2 \tau_{\delta}^{2}} \\
\Xi_{\alpha} & \equiv £_{\alpha} \kappa_{3}+\frac{£_{\alpha} £_{3} \kappa_{1}}{\tau_{\delta}}+\left(£_{3}\left(\tau_{\sigma}-3 \tau_{\delta}\right)-4 \kappa_{1} \kappa_{3}\right) \frac{£_{\alpha} \kappa_{1}}{2 \tau_{\delta}^{2}} .
\end{aligned}
$$

In these sub-branches, the rank of $\boldsymbol{R}_{[1,(11)]}^{\# 1}$ and $\boldsymbol{R}_{[1,(11)]}^{\# 2}$ controls the number of KVs in the same way as that presented in Sub-subsection 4.1.1.

4.1.3. Branch where the Segre type is $[1,11]$ In this branch, the eigenvalues $\left\{\lambda_{\alpha}\right\}$ differ from each other. Then, it immediately follows from eqs. (4.4) and (4.6) that

$$
\begin{aligned}
& \kappa_{3}=\delta \lambda_{1} \kappa_{2}, \\
& \kappa_{1}=-\delta \lambda_{2} \eta_{3}, \\
& \eta_{2}=-\delta \lambda_{3} \eta_{1}, \\
& \varpi_{1}=\eta_{1} \omega_{1}+\left(\tau_{2}-\tau_{3}\right) \omega_{2}, \\
& \varpi_{2}=-\kappa_{2} \omega_{2}+\left(\tau_{1}+\tau_{3}\right) \omega_{3} \text {, } \\
& \varpi_{3}=\left(\tau_{1}-\tau_{2}\right) \omega_{1}-\eta_{3} \omega_{3} \text {, }
\end{aligned}
$$


where $\delta \lambda_{1} \equiv\left(\lambda_{1}-\lambda_{2}\right) /\left(\lambda_{3}-\lambda_{1}\right), \delta \lambda_{2} \equiv\left(\lambda_{2}-\lambda_{3}\right) /\left(\lambda_{1}-\lambda_{2}\right)$ and $\delta \lambda_{3} \equiv\left(\lambda_{3}-\lambda_{1}\right) /\left(\lambda_{2}-\lambda_{3}\right)$. Given these conditions (4.21), the Killing equation (1.1) produces a PDE system of the form

$$
\nabla_{a} \boldsymbol{\omega}=\boldsymbol{\Omega}_{a} \boldsymbol{\omega}, \quad \boldsymbol{\omega} \equiv\left[\begin{array}{lll}
\omega_{1} & \omega_{2} & \omega_{3}
\end{array}\right]^{T},
$$

where

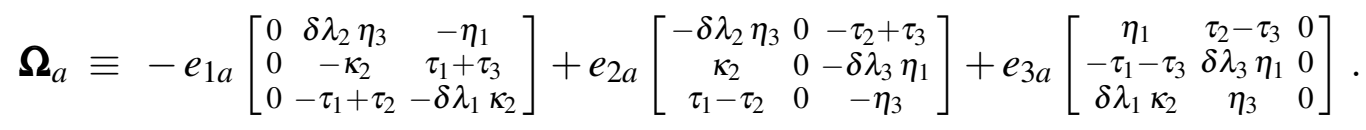

The compatibility condition for eq. (4.22) leads to

$$
\boldsymbol{R}_{[1,11]}=\left[\begin{array}{lll}
£_{1} \kappa_{1} & £_{2} \kappa_{1} & £_{3} \kappa_{1} \\
£_{1} \kappa_{2} & £_{2} \kappa_{2} & £_{3} \kappa_{2} \\
£_{1} \eta_{1} & £_{2} \eta_{1} & £_{3} \eta_{1} \\
£_{1} \tau_{1} & £_{2} \tau_{1} & £_{3} \tau_{1} \\
£_{1} \tau_{2} & £_{2} \tau_{2} & £_{3} \tau_{2} \\
£_{1} \tau_{3} & £_{2} \tau_{3} & £_{3} \tau_{3}
\end{array}\right]
$$

In this sub-branch, the rank of $\boldsymbol{R}_{[1,11]}$ governs the number of KVs in the same way as that presented in Sub-subsection 4.1.1.

4.1.4. Short summary of class 3 type $[1,11]$ Let us visually abridge the results obtained in this subsection in Figures 4b-4d.

\subsection{Type [21] and its degeneracy}

In this case, we have the following Jordan chains:

$$
\begin{aligned}
& S^{a}{ }_{b} j_{1}^{b}=\lambda_{1} j_{1}^{a}, \\
& S^{a}{ }_{b} j_{2}^{b}=\lambda_{1} j_{2}^{a}+j_{1}^{a}, \\
& S^{a}{ }_{b} j_{3}^{b}=\lambda_{3} j_{3}^{a},
\end{aligned}
$$

with $2 \lambda_{1}+\lambda_{3}=0$. Here $j_{\alpha}^{a}$ is an generalised eigenvector of $S_{b}^{a}$. It can be shown that the eigenvectors $j_{1}^{a}, j_{3}^{a}$ are respectively null and spacelike. The causal nature of $j_{2}$ is indeterminate. Taking the double-null basis $\left\{u^{a}, v^{a}, e^{a}\right\}$ of $T(M)$ as

$$
g^{a b}=u^{a} v^{b}+v^{a} u^{b}+e^{a} e^{b}
$$

it turns out useful to choose $u^{a} \equiv j_{1}^{a}$ and $e^{a} \equiv j_{3}^{a}$, with $v^{a}$ being a null vector such that $g_{a b} u^{a} v^{b}=1$ and $g_{a b} v^{a} e^{b}=0$. Then, any KV can be written as

$$
K^{a}=\omega_{u} u^{a}+\omega_{v} v^{a}+\omega_{e} e^{a},
$$

where scalars $\left\{\omega_{u}, \omega_{v}, \omega_{e}\right\}$ are yet indeterminate. 
type $[1,(11)]$

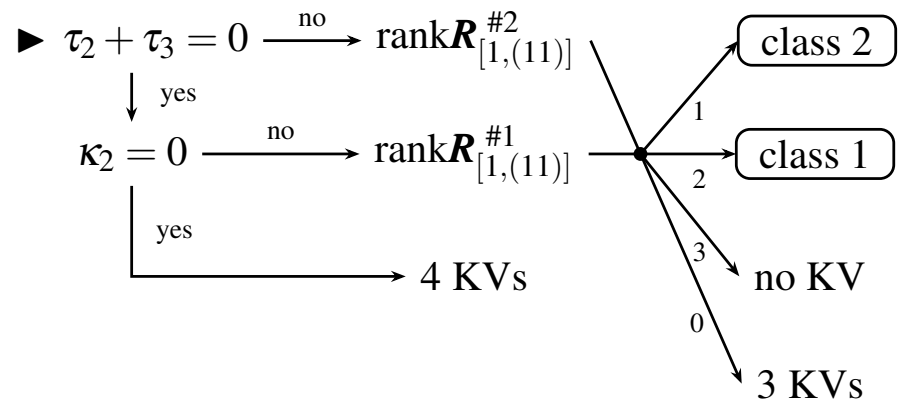

Figure 4b. The sub-sub-algorithm for the class 3 type $[1,(11)]$. See eqs. (4.12) and (4.13) for notations.

type $[(1,1) 1]$

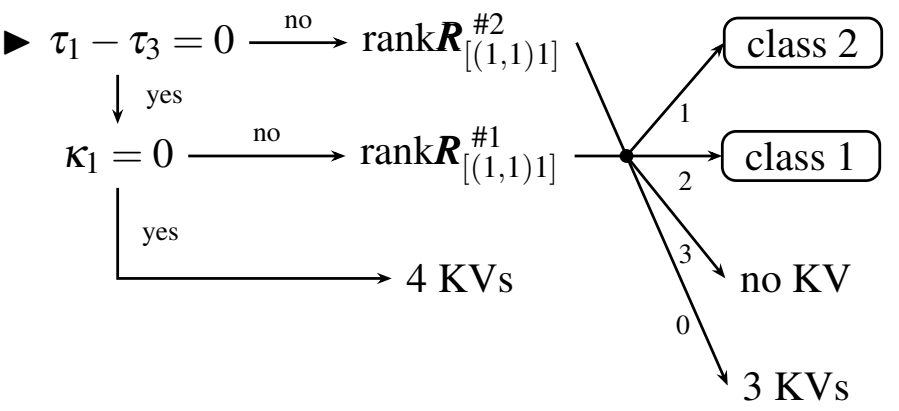

Figure 4c. The sub-sub-algorithm for the class 3 type $[(1,1) 1]$. See eqs. (4.19) and (4.20) for notations.

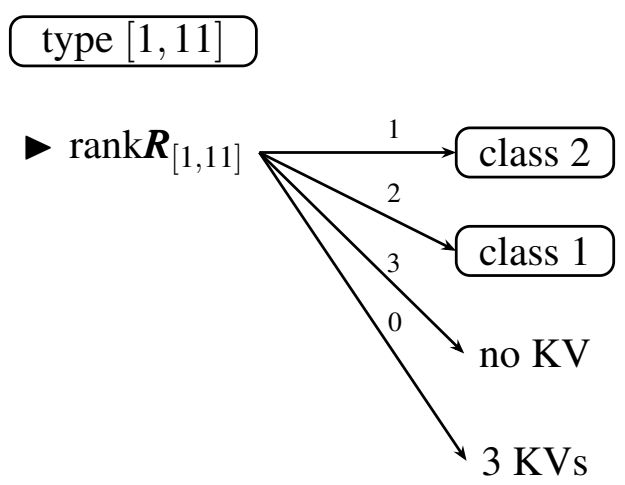

Figure 4d. The sub-sub-algorithm for the class 3 type $[1,11]$. See eq. (4.24) for notations. 
The first compatibility condition, $£_{K} S_{a b}=0$, of eq. (1.1) yields

$$
\begin{aligned}
\lambda \varpi_{e} & =-\lambda\left(\tau_{u}-\tau_{v}\right) \omega_{v}-\lambda \kappa_{e} \omega_{e} \\
3 \lambda \varpi_{u}-S_{v v} \varpi_{e} & =-3 \lambda\left(\tau_{u}+\tau_{e}\right) \omega_{u}-\left(3 \eta_{v} \lambda-\left(\tau_{u}-\tau_{v}\right) S_{v v}\right) \omega_{v}+\kappa_{e} S_{v v} \omega_{e} \\
2 S_{v v} \varpi_{v} & =-\left(£_{u} S_{v v}+2 \kappa_{u} S_{v v}\right) \omega_{u}-\left(£_{v} S_{v v}\right) \omega_{v}-\left(£_{e} S_{v v}-2\left(\tau_{v}-\tau_{e}\right) S_{v v}\right) \omega_{e}
\end{aligned}
$$

where $\lambda \equiv \lambda_{1}=-(1 / 2) \lambda_{3}$ and $S_{v v} \equiv S_{a b} v^{a} v^{b}$. The Ricci rotation coefficients are defined by eqs. (3.18), and the 1 -jet variables $\left\{\varpi_{u}, \varpi_{v}, \varpi_{e}\right\}$ are respectively defined as

$$
\varpi_{u} \equiv £_{e} \omega_{u}, \quad \varpi_{v} \equiv £_{v} \omega_{v}, \quad \varpi_{e} \equiv £_{u} \omega_{e}
$$

The second Bianchi identity $\nabla_{a} R_{b}^{a}-(1 / 2) \nabla_{b} R=0$ puts the following constraints

$$
\begin{aligned}
& 0=\lambda \kappa_{e} \\
& 0=£_{u} S_{v v}-3 \eta_{e} \lambda+\left(2 \kappa_{u}-\kappa_{e}\right) S_{v v} \\
& 0=\eta_{u} S_{v v}+3 \lambda\left(\tau_{u}+\tau_{v}\right)
\end{aligned}
$$

Suppose $S_{v v}=0$. Then eqs. (4.25) and (4.26) imply that the basis $\left\{u^{a}, v^{a}, e^{a}\right\}$ satisfies

$$
S^{a}{ }_{b} u^{b}=\lambda u^{a}, \quad S^{a}{ }_{b} v^{b}=\lambda v^{a}, \quad S_{b}^{a} e^{b}=-2 \lambda e^{a},
$$

which contradicts the assumption that the Segre type of $S^{a}{ }_{b}$ is [21]. It therefore follows that $S_{v v} \neq 0$. In the remaining parts of this subsection, we investigate the Segre types [(21)] and [21] separately.

4.2.1. Branch where the Segre type is $[(21)]$ In this branch, three eigenvalues of $S^{a}{ }_{b}$ are coincident and then $\lambda=\lambda_{1}=\lambda_{3}=0$ follows from traceless property. Then, eqs. (4.28) and (4.30) are combined to give

$$
\begin{aligned}
& \varpi_{v}=-\left(\frac{\kappa_{e}}{2}\right) \omega_{u}-\left(£_{v} \varphi\right) \omega_{v}-\left(£_{e} \varphi-\left(\tau_{v}-\tau_{e}\right)\right) \omega_{e} \\
& \varpi_{e}=-\left(\tau_{u}-\tau_{v}\right) \omega_{v}-\kappa_{e} \omega_{e}
\end{aligned}
$$

where $\varphi \equiv(1 / 2) \log S_{v v}$. Given these conditions (4.31), the Killing equation (1.1) and the identities $\nabla_{[a} \nabla_{b]} \omega_{u}=\nabla_{[a} \nabla_{b]} \omega_{v}=\nabla_{[a} \nabla_{b]} \omega_{e}=0$ produce a PDE system of the form

$$
\nabla_{a} \boldsymbol{\omega}=\boldsymbol{\Omega}_{a} \boldsymbol{\omega}, \quad \boldsymbol{\omega} \equiv\left[\begin{array}{llll}
\omega_{u} & \omega_{v} & \omega_{e} & \varpi_{u}
\end{array}\right]^{T},
$$


where

$$
\begin{aligned}
& \boldsymbol{\Omega}_{a} \equiv u_{a}\left[\begin{array}{cccc}
\kappa_{v} & 0 & \eta_{v} & 0 \\
-\frac{\kappa_{e}}{2} & -£_{v} \varphi & \tau_{v}-\tau_{e}-£_{e} \varphi & 0 \\
-\tau_{v}-\tau_{e} & -\eta_{v} & -\eta_{e} & -1 \\
\frac{\eta_{v}\left(2 \kappa_{u}+\kappa_{e}\right)}{2}-\kappa_{v}\left(\tau_{v}-\tau_{e}\right)+£_{e} \kappa_{v} & \eta_{v}\left(\eta_{e}+\kappa_{v}-£_{v} \varphi\right) & -\eta_{v}\left(\tau_{u}+\tau_{v}+£_{e} \varphi\right)+£_{e} \eta_{v} & \kappa_{v}+\eta_{e}
\end{array}\right] \\
& +v_{a}\left[\begin{array}{cccc}
-\frac{2 \kappa_{u}-\kappa_{e}}{2} & £_{\nu} \varphi-\kappa_{v} & £_{e} \varphi+\tau_{u}+\tau_{e} & 0 \\
0 & \kappa_{u} & 0 & 0 \\
0 & -\tau_{u}+\tau_{v} & -\kappa_{e} & 0 \\
\frac{\kappa_{e}\left(\tau_{u}-3 \tau_{e}\right)}{2}+\kappa_{u}\left(\tau_{u}+\tau_{e}\right)-£_{e} \kappa_{u} & \eta_{e}\left(\tau_{u}-\tau_{v}\right)-\left(\tau_{u}+\tau_{e}\right)\left(£_{\nu} \varphi-\kappa_{v}\right) & £_{e} \tau_{u}-\left(\tau_{u}+\tau_{e}\right)\left(£_{e} \varphi+\tau_{u}+\tau_{e}\right) & -\kappa_{u}
\end{array}\right] \\
& +e_{a}\left[\begin{array}{cccc}
0 & 0 & 0 & 1 \\
0 & -\tau_{v}+\tau_{e} & 0 & 0 \\
\kappa_{e} & \eta_{e} & 0 & 0 \\
\frac{\eta_{e}\left(\kappa_{e}-2 \kappa_{u}\right)}{2}+\tau_{u}^{2}-\tau_{e}^{2}-£_{u} \eta_{e}-£_{e}\left(\tau_{u}+\tau_{e}\right) & \eta_{v}\left(\tau_{u}+\tau_{v}-2 \tau_{e}\right)-£_{v} \eta_{e}-£_{e} \eta_{v}+\eta_{e} £_{v} \varphi & \eta_{e} £_{e} \varphi-£_{e} \eta_{e} & -2 \tau_{e}
\end{array}\right] \text {. }
\end{aligned}
$$

Several parts of the compatibility condition for eq. (4.32) lead to

$$
\begin{aligned}
\sigma \varpi_{u} & =-\left(\mathrm{e}^{\left.\varphi_{£_{u} \Sigma}+\left(\tau_{u}+\tau_{e}\right) \sigma\right) \omega_{u}-\left(\mathrm{e}^{\varphi} £_{v} \Sigma+\eta_{v} \sigma\right) \omega_{v}-\left(\mathrm{e}^{\varphi} £_{e} \Sigma\right) \omega_{e}},\right. \\
\kappa_{e} \varpi_{u} & =-\kappa_{e}\left(\tau_{v}+\tau_{e}\right) \omega_{u}-\left(£_{v} \tau_{v}+\eta_{v} \kappa_{e}\right) \omega_{v}-\left(£_{e} \tau_{v}\right) \omega_{e}
\end{aligned}
$$

where

$$
\sigma \equiv £_{e} \varphi+\tau_{v}+\tau_{e}, \quad \Sigma \equiv £_{v}\left(\mathrm{e}^{-\varphi}\right)+\kappa_{v} e^{-\varphi}
$$

This implies that $\varpi_{u}$ can be expressed in terms of $\left\{\omega_{u}, \omega_{v}, \omega_{e}\right\}$ except when $\sigma=\kappa_{e}=0$. Depending on the nonzeroness of the coefficients $\left\{\sigma, \kappa_{e}\right\}$, the analysis falls into three subbranches.

Sub-branch where $\sigma=\kappa_{e}=0 \quad$ In this sub-branch, the 1 -jet variable $\varpi_{u}$ cannot be expressed in terms of $\left\{\omega_{u}, \omega_{v}, \omega_{e}\right\}$. The remaining parts of the compatibility condition for eq. (4.32) read

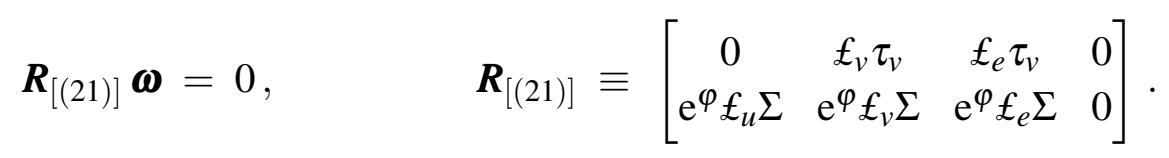

Remark that some remaining components are derivable from its derivative. In this sub-branch, the rank of $\boldsymbol{R}_{[(21)]}$ governs the number of KVs in the same way as that presented in Subsubsection 4.1.1.

Other sub-branches Except when $\sigma=\kappa_{e}=0$, eqs. (4.34) allow us to write the 1-jet variable $\varpi_{u}$ in terms of $\left\{\omega_{u}, \omega_{v}, \omega_{e}\right\}$. In these sub-branches, eq. (4.32) reduces to a PDE system of the form

$$
\nabla_{a} \boldsymbol{\omega}=\boldsymbol{\Omega}_{a} \boldsymbol{\omega}, \quad \boldsymbol{\omega} \equiv\left[\begin{array}{lll}
\omega_{u} & \omega_{v} & \omega_{e}
\end{array}\right]^{T}
$$


Here, the compatibility condition of eq. (4.36a) is considered collectively. The results are displayed as follows:

(\#1) For the case of $\sigma=0, \kappa_{e} \neq 0$,

$$
\begin{aligned}
& \varpi_{u}=-\left(\tau_{v}+\tau_{e}\right) \omega_{u}-\left(\frac{£_{v} \tau_{v}}{\kappa_{e}}+\eta_{v}\right) \omega_{v}-\left(\frac{£_{e} \tau_{v}}{\kappa_{e}}\right) \omega_{e} \\
& \boldsymbol{\Omega}_{a}=u_{a}\left[\begin{array}{ccc}
\kappa_{v} & 0 & \eta_{v} \\
-\frac{\kappa_{e}}{2} & -£_{v} \varphi & \tau_{v}-\tau_{e}-£_{e} \varphi \\
0 & \frac{f_{v} \tau_{v}}{\kappa_{e}} & \frac{f_{e} \tau_{v}}{\kappa_{e}}-\eta_{e}
\end{array}\right]+v_{a}\left[\begin{array}{ccc}
-\kappa_{u}+\frac{\kappa_{e}}{2} & £_{v} \varphi-\kappa_{v} & £_{e} \varphi+\tau_{u}+\tau_{e} \\
0 & \kappa_{u} & 0 \\
0 & -\tau_{u}+\tau_{v} & -\kappa_{e}
\end{array}\right] \\
& +e_{a}\left[\begin{array}{ccc}
-\tau_{v}-\tau_{e} & -\eta_{v}-\frac{f_{v} \tau_{v}}{\kappa_{e}} & -\frac{f_{e} \tau_{v}}{\kappa_{e}} \\
0 & \tau_{e}-\tau_{v} & 0 \\
\kappa_{e} & \eta_{e} & 0
\end{array}\right] \\
& \boldsymbol{R}_{[(21)]}^{\# 1}=\left[\begin{array}{ccc}
£_{u} \tau_{v} & £_{v} \tau_{v} & £_{e} \tau_{v} \\
\mathrm{e}^{\varphi} £_{u} \Sigma & \mathrm{e}^{\varphi £_{v} \Sigma} & \mathrm{e}^{\varphi £_{e} \Sigma} \\
£_{u} \kappa_{e}+\kappa_{e} £_{u} \varphi & £_{v} \kappa_{e}+\kappa_{e} £_{v} \varphi & £_{e} \kappa_{e}+\kappa_{e} £_{e} \varphi \\
£_{u} \eta_{e}-\eta_{e} £_{u} \varphi & £_{v} \eta_{e}-\eta_{e} £_{v} \varphi & £_{e} \eta_{e}-\eta_{e} £_{e} \varphi \\
£_{u} \eta_{v}-2 \eta_{v} £_{u} \varphi & £_{v} \eta_{v}-2 \eta_{v} £_{v} \varphi & £_{e} \eta_{v}-2 \eta_{v} £_{e} \varphi
\end{array}\right] .
\end{aligned}
$$

(\#2) For the case of $\sigma \neq 0$,

$$
\begin{aligned}
& \varpi_{u}=-\left(\frac{\mathrm{e}^{\varphi} £_{u} \Sigma}{\sigma}+\tau_{u}+\tau_{e}\right) \omega_{u}-\left(\frac{\mathrm{e}^{\varphi} £_{v} \Sigma}{\sigma}+\eta_{v}\right) \omega_{v}-\left(\frac{\mathrm{e}^{\varphi} £_{e} \Sigma}{\sigma}\right) \omega_{e},
\end{aligned}
$$

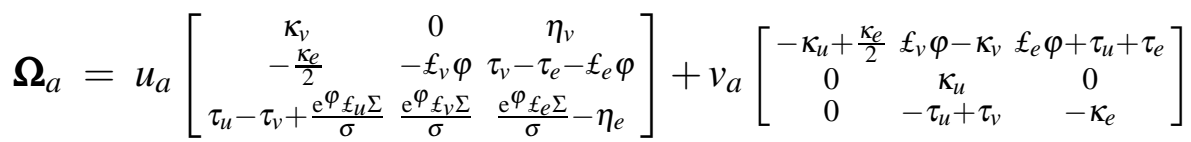

$$
\begin{aligned}
& +e_{a}\left[\begin{array}{ccc}
-\tau_{u}-\tau_{e}-\frac{\mathrm{e}^{\varphi} \varphi_{f_{u} \Sigma}}{\sigma} & -\eta_{v}-\frac{\mathrm{e}^{\varphi_{f_{v} \Sigma}}}{\sigma} & -\frac{\mathrm{e}^{\varphi_{f_{e} \Sigma}}}{\sigma} \\
0 & -\tau_{v}+\tau_{e} & 0 \\
\kappa_{e} & \eta_{e} & 0
\end{array}\right], \\
& \boldsymbol{R}_{[(21)]}^{\# 2}=\left[\begin{array}{ccc}
£_{u}\left(\sigma-\frac{5}{2} \tau_{v}\right) & £_{v}\left(\sigma-\frac{5}{2} \tau_{v}\right) & £_{e}\left(\sigma-\frac{5}{2} \tau_{v}\right) \\
£_{u} \tau_{v}-\frac{\mathrm{e}^{\varphi} \kappa_{e} £_{u} \Sigma}{\sigma} & £_{v} \tau_{v}-\frac{\mathrm{e}^{\varphi} \kappa_{e} £_{v} \Sigma}{\sigma} & £_{e} \tau_{v}-\frac{\mathrm{e}^{\varphi} \kappa_{e} £_{e} \Sigma}{\sigma} \\
£_{u} \kappa_{e}+\kappa_{e} £_{u} \varphi & £_{v} \kappa_{e}+\kappa_{e} £_{v} \varphi & £_{e} \kappa_{e}+\kappa_{e} £_{e} \varphi \\
\Phi_{u} & \Phi_{v} & \Phi_{e} \\
\Theta_{u} & \Theta_{v} & \Theta_{e}
\end{array}\right],
\end{aligned}
$$

where

$$
\begin{aligned}
& \Phi_{\alpha} \equiv £_{\alpha} \eta_{v}-2 \eta_{v} £_{\alpha} \varphi+\frac{\mathrm{e}^{\varphi} £_{\alpha} £_{v} \Sigma}{\sigma} \\
& -\frac{\mathrm{e}^{\varphi}\left(£_{\alpha} \varphi\right)\left(£_{v} \Sigma\right)}{\sigma}+\left(\mathrm{e}^{\varphi} £_{e} \Sigma-£_{v} \sigma+\left(£_{v} \varphi-\kappa_{v}-\eta_{e}\right) \sigma\right) \frac{\mathrm{e}^{\varphi} £_{\alpha} \Sigma}{\sigma^{2}}, \\
& \Theta_{\alpha} \equiv £_{\alpha} \eta_{e}-\eta_{e} £_{\alpha} \varphi-\frac{\mathrm{e}^{\varphi} £_{\alpha} £_{e} \Sigma}{\sigma}+\left(\mathrm{e}^{\varphi} £_{u} \Sigma+£_{e} \sigma+\left(\tau_{u}+\tau_{v}-\sigma\right) \sigma\right) \frac{\mathrm{e}^{\varphi} £_{\alpha} \Sigma}{\sigma^{2}},
\end{aligned}
$$

In these sub-branches, the rank of $\boldsymbol{R}_{[(21)]}^{\# 1}$ and $\boldsymbol{R}_{[(21)]}^{\# 2}$ governs the number of KVs in the same way as that presented in Sub-subsection 4.1.1. 
4.2.2. Branch where the Segre type is [21] In this branch, it is assumed that $\lambda=\lambda_{1}=$ $-(1 / 2) \lambda_{3} \neq 0$. Then, it immediately follows from eqs. (4.28) and (4.30) that

$$
\begin{aligned}
& \varpi_{u}=-\left(\tau_{u}+\tau_{e}\right) \omega_{u}-\eta_{v} \omega_{v}, \\
& \varpi_{v}=-\left(£_{u} \varphi+\kappa_{u}\right) \omega_{u}-\left(£_{v} \varphi\right) \omega_{v}-\left(£_{e} \varphi-\tau_{v}+\tau_{e}\right) \omega_{e}, \\
& \varpi_{e}=-\left(\tau_{u}-\tau_{v}\right) \omega_{v},
\end{aligned}
$$

and

$$
\kappa_{e}=0, \quad \eta_{u}=-\left(\frac{3 \lambda}{S_{v v}}\right)\left(\tau_{u}+\tau_{v}\right)
$$

Given these conditions (4.38), the Killing equation (1.1) produces a PDE system of the form

$$
\nabla_{a} \boldsymbol{\omega}=\boldsymbol{\Omega}_{a} \boldsymbol{\omega}, \quad \boldsymbol{\omega} \equiv\left[\begin{array}{lll}
\omega_{u} & \omega_{v} & \omega_{e}
\end{array}\right]^{T},
$$

where

$\boldsymbol{\Omega}_{a} \equiv u_{a}\left[\begin{array}{ccc}\kappa_{v} & 0 & \eta_{v} \\ -\kappa_{u}-£_{u} \varphi & -£_{v} \varphi-£_{e} \varphi+\tau_{v}-\tau_{e} \\ \tau_{u}-\tau_{v} & 0 & -\eta_{e}\end{array}\right]+v_{a}\left[\begin{array}{cccc}£_{u} \varphi £_{v} \varphi-\kappa_{v} & £_{e} \varphi+\tau_{u}+\tau_{e} \\ 0 & \kappa_{u} & -\frac{3 \lambda}{S_{v v}}\left(\tau_{u}+\tau_{v}\right) \\ 0 & -\tau_{u}+\tau_{v} & 0\end{array}\right]+e_{a}\left[\begin{array}{ccc}-\tau_{u}-\tau_{e} & -\eta_{v} & 0 \\ \frac{3 \lambda}{S_{v v}}\left(\tau_{u}+\tau_{v}\right)-\tau_{v}+\tau_{e} & 0 \\ 0 & \eta_{e} & 0\end{array}\right]$.

with $\varphi \equiv(1 / 2) \log S_{v v}$. The compatibility condition for eq. (4.39) leads to

$$
\boldsymbol{R}_{[21]}=\left[\begin{array}{ccc}
£_{u} \tau_{u} & £_{v} \tau_{u} & £_{e} \tau_{u} \\
£_{u} \tau_{v} & £_{v} \tau_{v} & £_{e} \tau_{v} \\
£_{u} \sigma & £_{v} \sigma & £_{e} \sigma \\
\mathrm{e}^{\varphi} £_{u} \Sigma & \mathrm{e}^{\varphi} £_{v} \Sigma & \mathrm{e}^{\varphi} £_{e} \Sigma \\
£_{u} \eta_{v}-2 \eta_{v} £_{u} \varphi & £_{v} \eta_{v}-2 \eta_{v} £_{v} \varphi & £_{e} \eta_{v}-2 \eta_{v} £_{e} \varphi \\
£_{u} \eta_{e}-\eta_{e} £_{u} \varphi & £_{v} \eta_{e}-\eta_{e} £_{v} \varphi & £_{e} \eta_{e}-\eta_{e} £_{e} \varphi
\end{array}\right]
$$

where

$$
\Sigma \equiv £_{v}\left(\mathrm{e}^{-\varphi}\right)+\kappa_{v} e^{-\varphi}
$$

In this sub-branch, the rank of $\boldsymbol{R}_{[21]}$ governs the number of KVs in the same way as that presented in Sub-subsection 4.1.1.

4.2.3. Short summary of class 3 type [21] We synopsise the results obtained in this subsection in Figures 4e-4f. 


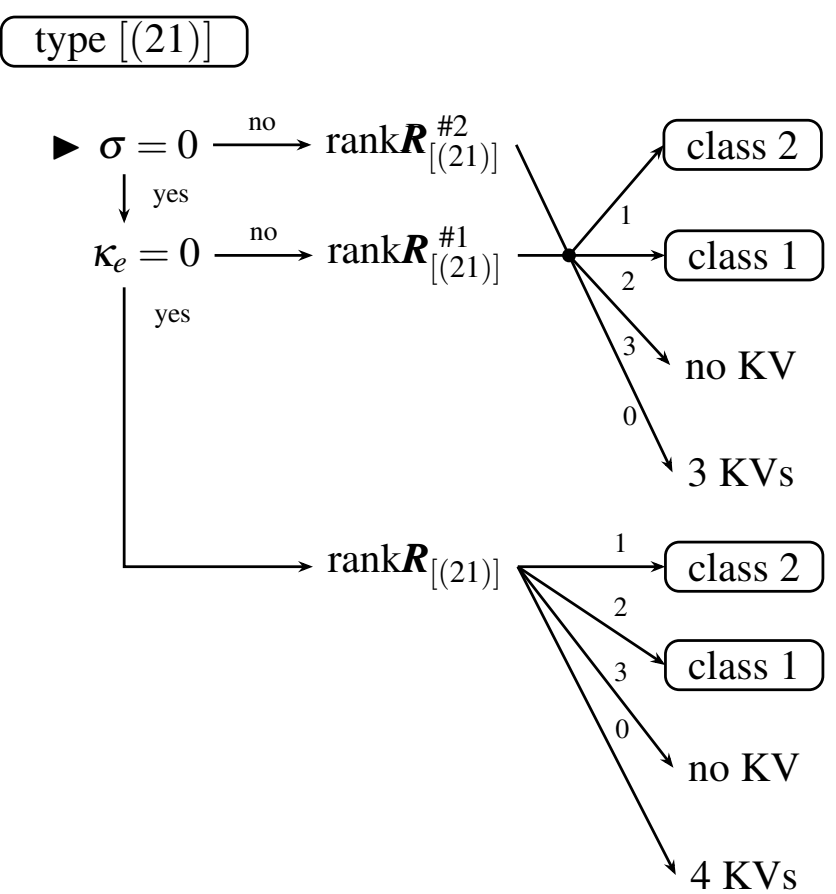

Figure 4e. The sub-sub-algorithm for the class 3 type $[(21)]$. See eqs. (4.35)-(4.37) for notations.

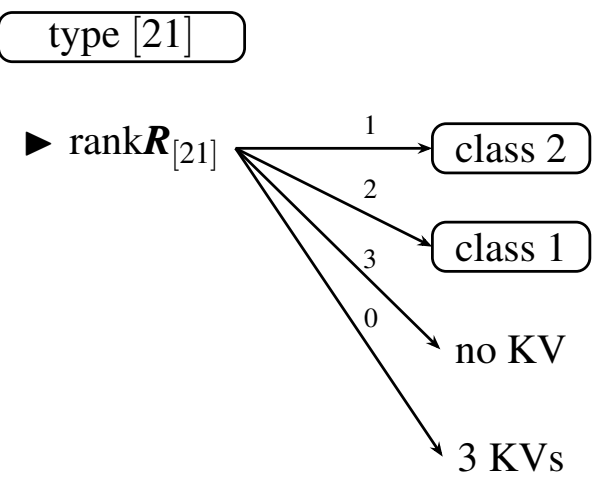

Figure 4f. The sub-sub-algorithm for the class 3 type [21]. See eq. (4.41a) for notations.

\subsection{Type [3]}

In this case, we have the following Jordan chain:

$$
\begin{aligned}
& S^{a}{ }_{b} j_{1}^{b}=\lambda j_{1}^{a}, \\
& S^{a}{ }_{b} j_{2}^{b}=\lambda j_{2}^{a}+j_{1}^{a}, \\
& S^{a}{ }_{b} j_{3}^{b}=\lambda j_{3}^{a}+j_{2}^{a},
\end{aligned}
$$

with $\lambda=0$. Here $j_{\alpha}^{a}$ is an generalised eigenvector of $S^{a}{ }_{b}$. It can be shown that the vectors $j_{1}^{a}, j_{2}^{a}$ are respectively null and spacelike, whereas the causal nature of $j_{3}^{a}$ is free from restriction. In this subsection, it is assumed that $\left\{u^{a}, v^{a}, e^{a}\right\}$ forms a double-null basis of 
$T(M)$

$$
g^{a b}=u^{a} v^{b}+v^{a} u^{b}+e^{a} e^{b},
$$

where $u^{a} \equiv j_{1}^{a} ; e^{a} \equiv j_{2}^{a} ; v^{a}$ is defined as a null vector such that $g_{a b} u^{a} v^{b}=1$ and $g_{a b} v^{a} e^{b}=0$. Then, any KV can be written as

$$
K^{a}=\omega_{u} u^{a}+\omega_{v} v^{a}+\omega_{e} e^{a},
$$

where scalars $\left\{\omega_{u}, \omega_{v}, \omega_{e}\right\}$ are yet indeterminate.

Calculating the second Bianchi identity, $\nabla_{a} R_{b}^{a}-(1 / 2) \nabla_{b} R=0$, leads to

$$
\eta_{u}=0, \quad \kappa_{u}=2 \kappa_{e}, \quad £_{u} S_{v v}+3 \kappa_{e} S_{v v}=2 \tau_{u}+\tau_{v}-\tau_{e},
$$

where $S_{v v} \equiv S_{a b} v^{a} v^{b}$ and the Ricci rotation coefficients are defined by eqs. (3.18). Note that $S_{a b} v^{a} e^{b}=1$ by eqs. (4.42) and (4.43). Using this identity, the first compatibility of eq. (1.1), $£_{K} S_{a b}=0$, can be written in components

$$
\begin{aligned}
& \varpi_{u}=\frac{1}{2}\left(\tau_{v}-3 \tau_{e}-3 \kappa_{e} S_{v v}\right) \omega_{u}+\frac{1}{2}\left(£_{v} S_{v v}-2 \eta_{v}\right) \omega_{v}+\frac{1}{2}\left(£_{e} S_{v v}\right) \omega_{e}, \\
& \varpi_{v}=-2 \kappa_{e} \omega_{u}+\left(\tau_{v}-\tau_{e}\right) \omega_{e}, \\
& \varpi_{e}=-\left(\tau_{u}-\tau_{v}\right) \omega_{v}-\kappa_{e} \omega_{e},
\end{aligned}
$$

where the 1-jet variables $\left\{\varpi_{u}, \varpi_{v}, \varpi_{e}\right\}$ are respectively defined as

$$
\varpi_{u} \equiv £_{e} \omega_{u}, \quad \varpi_{v} \equiv £_{v} \omega_{v}, \quad \varpi_{e} \equiv £_{u} \omega_{e} .
$$

Then, the Killing equation (1.1) produces a PDE system of the form

$$
\nabla_{a} \boldsymbol{\omega}=\boldsymbol{\Omega}_{a} \boldsymbol{\omega}, \quad \boldsymbol{\omega} \equiv\left[\begin{array}{lll}
\omega_{u} & \omega_{v} & \omega_{e}
\end{array}\right]^{T},
$$

where

$$
\begin{aligned}
\boldsymbol{\Omega}_{a} \equiv & u_{a}\left[\begin{array}{ccc}
\kappa_{v} & 0 & \eta_{v} \\
-2 \kappa_{e} & 0 & \tau_{v}-\tau_{e} \\
\frac{\tau_{e}-3 \tau_{v}+3 \kappa_{e} S_{v v}}{2} & -\frac{\epsilon_{v} S_{v v}}{2} & -\frac{\epsilon_{e} S_{v v}}{2}-\eta_{e}
\end{array}\right]+v_{a}\left[\begin{array}{ccc}
0 & -\kappa_{v} & \tau_{u}+\tau_{e} \\
0 & 2 \kappa_{e} & 0 \\
0 & -\tau_{u}+\tau_{v} & -\kappa_{e}
\end{array}\right] \\
& +e_{a}\left[\begin{array}{ccc}
\frac{\tau_{v}-3 \tau_{e}-3 \kappa_{e} S_{v v}}{2} & \frac{\varepsilon_{v} S_{v v}}{2}-\eta_{v} & \frac{f_{e} S_{v v}}{2} \\
0 & -\tau_{v}+\tau_{e} & 0 \\
\kappa_{e} & \eta_{e} & 0
\end{array}\right],
\end{aligned}
$$

The compatibility condition for eq. (4.48) leads to

$$
\boldsymbol{R}_{[3]}=\left[\begin{array}{ccc}
£_{u} \kappa_{e} & £_{v} \kappa_{e} & £_{e} \kappa_{e} \\
£_{u}\left(\tau_{e}-3 \tau_{v}\right) & £_{v}\left(\tau_{e}-3 \tau_{v}\right) & £_{e}\left(\tau_{e}-3 \tau_{v}\right) \\
£_{u}\left(\kappa_{e} S_{v v}+2 \tau_{v}\right) & £_{v}\left(\kappa_{e} S_{v v}+2 \tau_{v}\right) & £_{e}\left(\kappa_{e} S_{v v}+2 \tau_{v}\right) \\
£_{u} \kappa_{v}+\frac{\tau_{v}+\tau_{e}}{2} £_{u} S_{v v} & £_{v} \kappa_{v}+\frac{\tau_{v}+\tau_{e}}{2} £_{v} S_{v v} & £_{e} \kappa_{v}+\frac{\tau_{v}+\tau_{e}}{2} £_{e} S_{v v} \\
\Xi_{u} & \Xi_{v} & \Xi_{e} \\
\Theta_{u} & \Theta_{v} & \Theta_{e}
\end{array}\right]
$$

where

$$
\begin{aligned}
& \Xi_{\alpha} \equiv £_{\alpha} \eta_{e}+\frac{£_{\alpha} £_{e} S_{v v}}{2}+\left(\tau_{v}+\tau_{e}-3 \kappa_{e} S_{v v}\right) \frac{£_{\alpha} S_{v v}}{4}, \\
& \Theta_{\alpha} \equiv £_{\alpha} \eta_{v}-\frac{£_{\alpha} £_{v} S_{v v}}{2}+\left(£_{e} S_{v v}+2 \kappa_{v}+2 \eta_{e}\right) \frac{£_{\alpha} S_{v v}}{4} .
\end{aligned}
$$

In this sub-branch, the rank of $\boldsymbol{R}_{[3]}$ governs the number of KVs in the way shown in Figure 4g. 


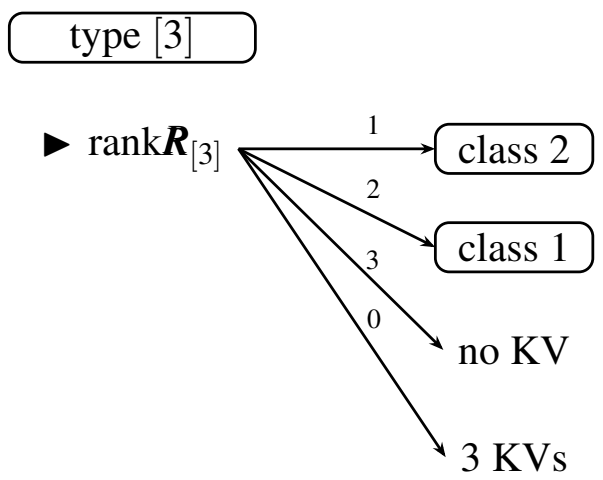

Figure 4g. The sub-sub-algorithm for the class 3 type [3]. See eq. (4.50a) for notations.

\subsection{Type $[z \bar{z} 1]$}

In this case, we have the following Jordan chains:

$$
\begin{aligned}
S^{a}{ }_{b} j_{+}^{b} & =\lambda_{+} j_{+}^{a}, \\
S^{a}{ }_{b} j_{-}^{b} & =\lambda_{-} j_{-}^{a}, \\
S^{a}{ }_{b} j^{b} & =\lambda j^{a},
\end{aligned}
$$

where $\lambda_{ \pm}=\alpha \pm i \beta(\beta \neq 0)$ are complex eigenvalues corresponding to the complex eigenvectors $j_{ \pm}^{a}=x^{a} \pm i y^{a}$. It follows from the symmetric traceless property of $S_{a b}$ that $2 \alpha+\lambda=0$ and $g_{a b}\left(x^{a} x^{b}+y^{a} y^{b}\right)=0$. On the other hand, the real/imaginary parts of eqs. (4.51) give

$$
\begin{aligned}
& S^{a}{ }_{b} x^{b}=\alpha x^{a}-\beta y^{a}, \\
& S^{a}{ }_{b} y^{b}=\beta x^{a}+\alpha y^{a} .
\end{aligned}
$$

This implies that the real vectors $\left\{x^{a}, y^{a}\right\}$ span a timelike surface. One can then fix $x^{a}$ to be timelike and $y^{a}$ to be spacelike without loss of generality. In this subsection, it is supposed that $\left\{e_{\alpha}^{a}\right\}$ forms an orthonormal basis of $T(M)$,

$$
g^{a b}=-e_{1}^{a} e_{1}^{b}+e_{2}^{a} e_{2}^{b}+e_{3}^{a} e_{3}^{b},
$$

where $e_{1}^{a} \propto x^{a} ; e_{2}^{a} \propto y^{a}$ and $e_{3}^{a} \propto j^{a}$. Then, any $\mathrm{KV}$ can be written as

$$
K^{a}=\sum_{\alpha=1}^{3} \omega_{\alpha} e_{\alpha}^{a},
$$

where scalars $\left\{\omega_{\alpha}\right\}$ are yet indeterminate.

The first compatibility condition, $£_{K} S_{a b}=0$, of eq. (1.1), gives rise to

$$
\begin{aligned}
& \varpi_{1}=\eta_{1} \omega_{1}+\left(\tau_{2}-\tau_{3}\right) \omega_{2}, \\
& \varpi_{2}=-\kappa_{2} \omega_{2}+\left(\tau_{1}+\tau_{3}\right) \omega_{3}, \\
& \varpi_{3}=\left(\tau_{1}-\tau_{2}\right) \omega_{1}-\eta_{3} \omega_{3},
\end{aligned}
$$


where the Ricci rotation coefficients are defined by eqs. (3.5). The 1-jet variables $\left\{\varpi_{u}, \varpi_{v}, \varpi_{e}\right\}$ are respectively defined as

$$
\varpi_{1} \equiv £_{3} \omega_{1}, \quad \varpi_{2} \equiv £_{1} \omega_{2}, \quad \varpi_{3} \equiv £_{2} \omega_{3} .
$$

Then, the Killing equation (1.1) produces a PDE system of the form

$$
\nabla_{a} \boldsymbol{\omega}=\boldsymbol{\Omega}_{a} \boldsymbol{\omega}, \quad \boldsymbol{\omega} \equiv\left[\begin{array}{lll}
\omega_{1} & \omega_{2} & \omega_{3}
\end{array}\right]^{T},
$$

where

$$
\boldsymbol{\Omega}_{a} \equiv-e_{1 a}\left[\begin{array}{ccc}
0 & -\kappa_{1} & -\eta_{1} \\
0 & -\kappa_{2} & \tau_{1}+\tau_{3} \\
0 & \tau_{2}-\tau_{1} & -\kappa_{3}
\end{array}\right]+e_{2 a}\left[\begin{array}{ccc}
\kappa_{1} & 0 & \tau_{3}-\tau_{2} \\
\kappa_{2} & 0 & \eta_{2} \\
\tau_{1}-\tau_{2} & 0 & -\eta_{3}
\end{array}\right]+e_{3 a}\left[\begin{array}{ccc}
\eta_{1} & \tau_{2}-\tau_{3} & 0 \\
-\tau_{1}-\tau_{3} & -\eta_{2} & 0 \\
\kappa_{3} & \eta_{3} & 0
\end{array}\right]
$$

where the Ricci rotation coefficients are defined by eqs. (3.5). Note that the second Bianchi identity imposes

$$
\eta_{3}-2 \kappa_{1}=\frac{3 \alpha}{\beta} \kappa_{3}, \quad \kappa_{3}+2 \kappa_{2}=-\frac{3 \alpha}{\beta} \eta_{3}, \quad \tau_{1}+\tau_{2}=\frac{3 \alpha}{\beta}\left(\eta_{1}-\eta_{2}\right),
$$

The compatibility condition for eq. (4.56) leads to

$$
\boldsymbol{R}_{[z \bar{z} 1]}=\left[\begin{array}{lll}
£_{1} \kappa_{3} & £_{2} \kappa_{3} & £_{3} \kappa_{3} \\
£_{1} \eta_{1} & £_{2} \eta_{1} & £_{3} \eta_{1} \\
£_{1} \eta_{2} & £_{2} \eta_{2} & £_{3} \eta_{2} \\
£_{1} \eta_{3} & £_{2} \eta_{3} & £_{3} \eta_{3} \\
£_{1} \tau_{2} & £_{2} \tau_{2} & £_{3} \tau_{2} \\
£_{1} \tau_{3} & £_{2} \tau_{3} & £_{3} \tau_{3}
\end{array}\right] .
$$

In this sub-branch, the rank of $\boldsymbol{R}_{[z \bar{z} 1]}$ governs the number of $\mathrm{KVs}$ in the way shown in Figure 4h.

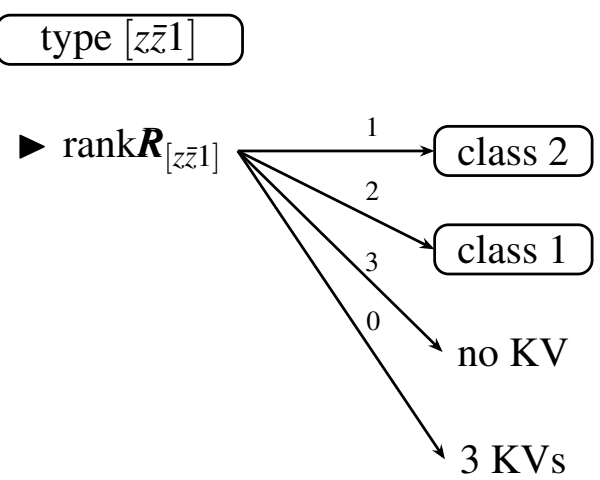

Figure 4h. The sub-sub-algorithm for the class 3 type $[z \bar{z} 1]$. See eq. (4.59) for notations. 


\section{Application}

In this section, a couple of examples is provided to illustrate how useful our prescription is. First, we shall consider the Lifshitz spacetime (see e.g. [18] and references therein) in Subsection 5.1, whose metric has an arbitrary constant $z$. Afterwards, we deal with a ppwave spacetime in Subsection 5.2, which serves as a typical example of vanishing scalar invariant spaces and is characterised by a single function $h$. As we will see below, a complete classification of their local isometry groups depends respectively on the values of $z$ and the profile of $h$. This demonstrates the power of the present formulation.

\subsection{The Lifshitz spacetime}

In condensed matter systems, many phase transitions are governed by the fixed points admitting the anisotropic dynamical scaling. In light of holography, a great deal of attention has been recently focused on the gravity dual with this dynamical scaling, which is modelled by the Lifshitz metric [18]. The three-dimensional Lifshitz metric reads

$$
g_{\text {Lifshitz }}=-\frac{r^{2 z}}{L^{2 z}} \mathrm{~d} t^{2}+\frac{L^{2}}{r^{2}} \mathrm{~d} r^{2}+\frac{r^{2}}{L^{2}} \mathrm{~d} x^{2}
$$

where $z$ is an arbitrary constant corresponding to the dynamical exponent and $L$ is related to the curvature scale. For this metric, the scalar curvature $R$ and the principal traces of of powers of the traceless Ricci operator $S^{a}{ }_{b}$ are all constants, which evaluate to

$$
R=-\frac{2\left(z^{2}+z+1\right)}{L^{2}}, \quad S^{(2)}=\frac{2(z-1)^{2}\left(z^{2}+z+1\right)}{3 L^{4}}, \quad S^{(3)}=\frac{(z-1)^{4}\left(2 z^{2}+5 z+2\right)}{9 L^{6}} .
$$

As the metric (5.1) belongs to the class 3, the criteria in Figure 4a must be checked. After simple calculations, we find that $S^{a}{ }_{b}=0$ if $z=1$. It then follows from the result in Figure 4a that $6 \mathrm{KVs}$ exist and the AdS metric is recovered. By solving the Killing equation (1.1), their explicit form can be read as

$$
\begin{aligned}
& \partial_{t}, \quad \partial_{x}, \quad x \partial_{t}+t \partial_{x}, \quad t \partial_{t}-r \partial_{r}+x \partial_{x}, \\
& \frac{L^{4}+r^{2}\left(t^{2}+x^{2}\right)}{2 r^{2}} \partial_{t}-r t \partial_{r}+t x \partial_{x}, \quad t x \partial_{t}-r x \partial_{r}-\frac{L^{4}-r^{2}\left(t^{2}+x^{2}\right)}{2 r^{2}} \partial_{x} .
\end{aligned}
$$

If $z=0$, the Segre type of $S^{a}{ }_{b}$ is $[1,(11)]$. Consequently, the number of KVs can be computed by the algorithm described in Figure $4 \mathrm{~b}$ and it equals 4 . One sees that the metric culminates in $\mathbb{R} \times H^{2}$. Once again, solving eq. (1.1) gives their explicit form

$$
\partial_{t}, \quad \quad \quad \quad \partial_{x}, \quad r x \partial_{r}+\frac{L^{4}-r^{2} x^{2}}{2 r^{2}} \partial_{x} .
$$

If $z \neq 0,1$, the Segre type of $S^{a}{ }_{b}$ is either $[(1,1) 1]$ for $z=-1$ or $[1,11]$ for $z \neq-1$. Then the number of KVs can be computed by the algorithm described in Figure 4c or 4d. In either case, there are $3 \mathrm{KVs}$ in the form

$$
\partial_{t}, \quad \partial_{x}, \quad t z \partial_{t}-r \partial_{r}+x \partial_{x}
$$


The last one captures the anisotropic scaling $t \rightarrow \lambda^{z} t, r \rightarrow \lambda^{-1} r, x \rightarrow \lambda x$. This completes a classification of the metric (5.1) based on their level of symmetry, which is summarised in Figure 5a.

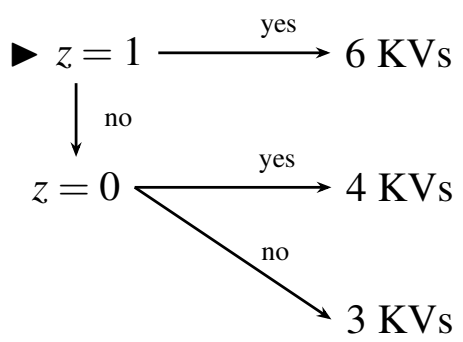

Figure 5a. A flowchart to classify the number of KVs of the Lifshitz spacetime (5.1) in 3D.

\subsection{The pp-wave spacetime}

For Lorentzian manifolds, a natural question to ask is whether our theorem works for the metric with vanishing scalar invariant (VSI) property. Here we show that it does.

A VSI spacetime is a Lorentzian manifold $M$ in which scalar Weyl invariants of any order vanish identically, yet the Riemann-Christoffel tensor $R_{a b c}{ }^{d}$ is nonvanishing. Note that scalar Weyl invariants (or polynomial curvature invariants) of order $p$ are scalars on $M$ obtained from the first $p$ covariant derivatives of the Riemann-Christoffel tensor $\nabla_{a_{1}} \cdots \nabla_{a_{p}} R_{b c d}{ }^{e}$ by tensor products and complete contractions [19]. There are nontrivial spacetimes with a VSI property which have received some attention in the context of general relativity, see e.g. [20, 21, 22].

As a classical example of VSI spacetimes, we deal with a pp-wave spacetime which admits a covariantly constant null Killing vector $V^{a}$ satisfying $\nabla_{a} V^{b}=0, V_{a} V^{a}=0$. In dimension 3 , the general form of the pp-wave metric takes the following form

$$
g_{\mathrm{pp}}=h(u, x) \mathrm{d} u^{2}+2 \mathrm{~d} u \mathrm{~d} v+\mathrm{d} x^{2},
$$

where $h$ is a function of $u$ and $x$. It is obvious that the covariantly constant null vector is given by $V=K_{1}=\partial_{v}$. Our aim here is to obtain a complete classification of KVs of $g_{\text {pp }}$ based on the tournure of the function $h$.

By the definition of VSI spacetimes, the metric (5.6) belongs to the class 3. After simple calculations, we find that the Segre type of the traceless Ricci operator $S^{a}{ }_{b}$ depends on whether the function $h$ satisfies a PDE $h_{, x x}=\frac{\partial^{2} h}{\partial x^{2}}=0$ or not. If $h_{, x x}=0$ holds, the Segre type is $[(1,11)]$ and $h$ takes the form

$$
h(u, x)=h_{0}(u)+2 x h_{1}(u),
$$

where $h_{0}$ and $h_{1}$ are arbitrary functions of $u$. Consequently it follows from the result in Figure 4a that $6 \mathrm{KVs}$ exist and the spacetime is locally reduced to the Minkowski $\mathbb{R}^{1,2}$. By solving 
the Killing equation (1.1), the explicit expressions of the set of KVs can be written as

$$
\begin{aligned}
K_{1}= & \partial_{v} \\
K_{2}= & H_{1} \partial_{v}-\partial_{x} \\
K_{3}= & \left(x+u H_{1}-\mathscr{H}_{1}\right) \partial_{v}-u \partial_{x} \\
K_{4}= & \partial_{u}-\frac{1}{2}\left(h+H_{1}^{2}\right) \partial_{v}+H_{1} \partial_{x} \\
K_{5}= & u \partial_{u}-\frac{1}{2}\left(2 v+u h+H+u H_{1}^{2}-\int \mathrm{d} u H_{1}^{2}\right) \partial_{v}+u H_{1} \partial_{x} \\
K_{6}= & \left(x-\mathscr{H}_{1}\right) \partial_{u}+\frac{1}{2}\left(\left(x+\mathscr{H}_{1}\right) H_{1}^{2}-\left(x-\mathscr{H}_{1}\right) h+2 v H_{1}+H_{0} H_{1}-H_{1} \int \mathrm{d} u H_{1}^{2}\right) \partial_{v} \\
& -\frac{1}{2}\left(H_{0}+2 H_{1} \mathscr{H}_{1}-\int \mathrm{d} u H_{1}^{2}+2 v\right) \partial_{x}
\end{aligned}
$$

where

$$
\begin{array}{rlrl}
H(u, x) & \equiv \int \mathrm{d} u h(u, x), & H_{0}(u) & \equiv \int \mathrm{d} u h_{0}(u), \\
H_{1}(u) & \equiv \int \mathrm{d} u h_{1}(u), & \mathscr{H}_{1}(u) \equiv \int \mathrm{d} u H_{1}(u) .
\end{array}
$$

The nonvanishing commutation relations for these KVs are

$$
\begin{array}{llll}
{\left[K_{1}, K_{5}\right]=-K_{1},} & {\left[K_{1}, K_{6}\right]=K_{2},} & {\left[K_{2}, K_{3}\right]=-K_{1},} \\
{\left[K_{2}, K_{6}\right]=-K_{4},} & {\left[K_{3}, K_{4}\right]=-K_{2},} & {\left[K_{3}, K_{5}\right]=-K_{3},} \\
{\left[K_{3}, K_{6}\right]=-K_{5},} & {\left[K_{4}, K_{5}\right]=K_{4},} & {\left[K_{5}, K_{6}\right]=-K_{6} .}
\end{array}
$$

These KVs constitute the 3-dimensional Poincaré algebra.

If $h_{, x x}=0$ fails to be fulfilled, the Segre type of $S_{b}^{a}$ is $[(21)]$ with the invariants $\left\{\sigma, \kappa_{e}\right\}=\left\{\frac{h_{, x x x}}{2 h_{, x x}}, 0\right\}$ (see Figure $4 \mathrm{e}$ and eq. (4.34) for notations). The number of KVs is controlled by either $\operatorname{rank} \boldsymbol{R}_{[(21)]}^{\# 2}$ if $h_{, x x x} \neq 0$ or $\operatorname{rank} \boldsymbol{R}_{[(21)]}$ if $h_{, x x x}=0$. In either case, any KV can be identified at the outset $K^{a}=\omega_{u} u^{a}+\omega_{v} v^{a}+\omega_{e} e^{a}$, where $\left\{u^{a}, v^{a}, e^{a}\right\}$ is the double-null basis defined as

$$
u^{a} \equiv\left(\partial_{v}\right)^{a}, \quad v^{a} \equiv\left(\partial_{u}\right)^{a}-\frac{h}{2}\left(\partial_{v}\right)^{a}, \quad e^{a} \equiv\left(\partial_{x}\right)^{a},
$$

whose nonvanishing rotation coefficient consists only of $\eta_{v}=-h_{, x} / 2$.

5.2.1. The case $\sigma \propto h_{, x x x}=0$ The solution to the equation $h_{, x x x}=0$ leads to the general form of $h$ as

$$
h(u, x)=h_{0}(u)+2 x h_{1}(u)+x^{2} h_{2}(u),
$$

where $\left\{h_{0}, h_{1}, h_{2}\right\}$ are arbitrary functions of $u$. By combining eqs. (5.11) and (5.10), the obstruction matrix $\boldsymbol{R}_{[(21)]}$ acting on $\boldsymbol{\omega}=\left[\omega_{u}, \omega_{v}, \omega_{e}, \varpi_{u}\right]^{T}$ reads

$$
\boldsymbol{R}_{[(21)]}=\left[\begin{array}{cccc}
0 & £_{v} \tau_{v} & £_{e} \tau_{v} & 0 \\
\mathrm{e}^{\varphi} £_{u} \Sigma & \mathrm{e}^{\varphi} £_{v} \Sigma & \mathrm{e}^{\varphi} £_{e} \Sigma & 0
\end{array}\right] \propto\left[\begin{array}{cccc}
0 & 0 & 0 & 0 \\
0 & \left(h_{, x x u} /\left(h_{, x x}\right)^{3 / 2}\right)_{, u} & 0 & 0
\end{array}\right] .
$$


Thereby $\operatorname{rank} \boldsymbol{R}_{[(21)]}=0$ if $h_{2}$ solves the $\operatorname{ODE}\left(h_{, x x u} /\left(h_{, x x}\right)^{3 / 2}\right)_{, u}=\left(h_{2, u} / h_{2}^{3 / 2}\right)_{, u}=0$ whose solution is given by $h_{2}(u)=1 /\left(c_{1} u+c_{2}\right)^{2}$, where $\left\{c_{1}, c_{2}\right\}$ are constants. Notice that a coordinate shift $u \rightarrow u+c_{0}\left(c_{0}=\right.$ const.) allows us to classify the solution as either $h_{2}(u)=$ const. for $c_{1}=0$ or $h_{2}(u) \propto u^{-2}$ for $c_{1} \neq 0$.

For the former case $h_{2}(u)=c=$ const. $\neq 0$, there are $4 \mathrm{KVs}$ in the form

$$
\begin{aligned}
K_{1}= & \partial_{v}, \\
K_{2}= & \left(\sqrt{c} x \mathrm{e}^{-\sqrt{c} u}-\left\langle h_{1}\right\rangle^{-}\right) \partial_{v}+\mathrm{e}^{-\sqrt{c} u} \partial_{x}, \\
K_{3}= & \left(\sqrt{c} x \mathrm{e}^{\sqrt{c} u}+\left\langle h_{1}\right\rangle^{+}\right) \partial_{v}-\mathrm{e}^{\sqrt{c} u} \partial_{x}, \\
K_{4}= & 2 \partial_{u}-\left(h_{0}+2 x h_{1}+\sqrt{c} x\left(\mathrm{e}^{\sqrt{c} u}\left\langle h_{1}\right\rangle^{-}-\mathrm{e}^{-\sqrt{c} u}\left\langle h_{1}\right\rangle^{+}\right)+\left\langle h_{1}\right\rangle^{+}\left\langle h_{1}\right\rangle^{-}\right) \partial_{v} \\
& +\left(\mathrm{e}^{\sqrt{c} u}\left\langle h_{1}\right\rangle^{-}+\mathrm{e}^{-\sqrt{c} u}\left\langle h_{1}\right\rangle^{+}\right) \partial_{x},
\end{aligned}
$$

where we have assumed $c>0$ and defined

$$
\left\langle h_{1}\right\rangle^{ \pm} \equiv \int \mathrm{d} u \mathrm{e}^{ \pm \sqrt{c} u} h_{1} .
$$

The nonzero commutators for these KVs are

$$
\left[K_{2}, K_{3}\right]=2 \sqrt{c} K_{1}, \quad\left[K_{2}, K_{4}\right]=2 \sqrt{c} K_{2}, \quad\left[K_{3}, K_{4}\right]=-2 \sqrt{c} K_{3} .
$$

These correspond to the $\mathfrak{s l}(2, \mathbb{R})$ algebra. One can deduce the explicit expressions of $\mathrm{KVs}$ also for the $c<0$ case.

For the latter case $h_{2}(u)=c u^{-2}(c=$ const. $\neq 0), 4 \mathrm{KVs}$ exist in the form

$$
\begin{aligned}
K_{1}= & \partial_{v} \\
K_{2}= & \left(2 c x u^{-\frac{1+k}{2}}-(1+k)\left\langle h_{1}\right\rangle_{1-k}\right) \partial_{v}+(1+k) u^{\frac{1-k}{2}} \partial_{x} \\
K_{3}= & \left(2 c x u^{-\frac{1-k}{2}}-(1-k)\left\langle h_{1}\right\rangle_{1+k}\right) \partial_{v}+(1-k) u^{\frac{1+k}{2}} \partial_{x} \\
K_{4}= & 2 k u \partial_{u}-\left(2 k v+x\left(2 k u h_{1}+\frac{1}{2}(1+k)^{2} u^{\frac{k-1}{2}}\left\langle h_{1}\right\rangle_{1-k}-\frac{1}{2}(1-k)^{2} u^{-\frac{k+1}{2}}\left\langle h_{1}\right\rangle_{1+k}\right)\right. \\
& \left.+k\left(u h_{0}+H_{0}\right)+(1+k) \int \mathrm{d} u u^{\frac{1+k}{2}} h_{1}\left\langle h_{1}\right\rangle_{1-k}-(1-k) \int \mathrm{d} u u^{\frac{1-k}{2}} h_{1}\left\langle h_{1}\right\rangle_{1+k}\right) \partial_{v} \\
& +\left((1+k) u^{\frac{1+k}{2}}\left\langle h_{1}\right\rangle_{1-k}-(1-k) u^{\frac{1-k}{2}}\left\langle h_{1}\right\rangle_{1+k}\right) \partial_{x}
\end{aligned}
$$

where we have used abbreviations

$$
k \equiv \sqrt{1+4 c}, \quad H_{0} \equiv \int \mathrm{d} u h_{0}, \quad\left\langle h_{1}\right\rangle_{p} \equiv \int \mathrm{d} u u^{\frac{p}{2}} h_{1} .
$$

In the above expressions, we have tentatively assumed $c>-1 / 4$. The commutation relations are given by

$$
\begin{array}{lll}
{\left[K_{1}, K_{4}\right]=-2 k K_{1},} & {\left[K_{2}, K_{4}\right]=-k(1-k) K_{2},} \\
{\left[K_{2}, K_{3}\right]=4 c k K_{1},} & {\left[K_{3}, K_{4}\right]=-k(1+k) K_{3} .}
\end{array}
$$


The KVs for the $c=-1 / 4$ and $c<-1 / 4$ cases can be obtained in a similar fashion, but we shall not attempt to do this here.

If $\sigma \propto h_{, x x x}=0$ but $\Sigma_{, u} \propto\left(h_{, x x u} /\left(h_{, x x}\right)^{3 / 2}\right)_{, u} \neq 0$, it follows from eq. (5.12) that $\operatorname{rank} \boldsymbol{R}_{[(21)]}=1$ and $\omega_{v}$ has to be zero. As any KV takes the form $K^{a}=\omega_{u} u^{a}+\omega_{e} e^{a}$, the results in Section 3.2 are reusable. Since all the spin coefficients are vanishing except for $\eta_{v}$, $\operatorname{rank} \boldsymbol{R}_{\mathrm{cls} .2}$ governs the number of KVs (see Figure 3c). For the function $h$ in the form of eq. (5.11), one can verify that $\operatorname{rank} \boldsymbol{R}_{\text {cls.2 }}$ is always zero. By solving eq. (1.1) directly, it can be ascertained that $3 \mathrm{KVs}$ exist in the form

$$
K_{1}=\partial_{v}, \quad K_{ \pm}=-\left(\int \mathrm{d} u h_{1} \phi_{ \pm}+x \int \mathrm{d} u h_{2} \phi_{ \pm}\right) \partial_{v}+\phi_{ \pm} \partial_{x}
$$

where $\phi_{ \pm}(u)$ are the two linearly independent solutions to the following ODE

$$
\phi_{, u u}=h_{2} \phi .
$$

By the conservation of Wronskian $\phi_{+, u} \phi_{-}-\phi_{+} \phi_{-, u}=$ const. $\equiv W$, the only nonvanishing commutator is

$$
\left[K_{+}, K_{-}\right]=W K_{1}
$$

5.2.2. The case $\sigma \propto h_{, x x x} \neq 0 \quad$ For the case in question, the obstruction matrix $\boldsymbol{R}_{[(21)]}^{\# 2}$ controls the number of KVs primarily (see Figure 4e). As a strategy for the classification, we focus on the first row of $\boldsymbol{R}_{[[(21)]}^{\# 2}$

$$
\left[£_{u}\left(\sigma-\frac{5}{2} \tau_{v}\right) £_{v}\left(\sigma-\frac{5}{2} \tau_{v}\right) £_{e}\left(\sigma-\frac{5}{2} \tau_{v}\right)\right] \propto\left[\begin{array}{lll}
0 & \left(h_{, x x x} / h_{, x x}\right)_{, u} & \left(h_{, x x x} / h_{, x x}\right)_{, x}
\end{array}\right] .
$$

It can be shown that if $\left(h_{, x x x} / h_{, x x}\right)_{, u}=\left(h_{, x x x} / h_{, x x}\right)_{, x}=0$ all entries of $\boldsymbol{R}_{[(21)]}^{\# 2}$ are zero except for the fifth row

$$
\left[\begin{array}{lll}
\Phi_{u} & \Phi_{v} & \Phi_{e}
\end{array}\right] \propto\left[\begin{array}{lll}
0 & \varsigma h_{, x x u}-\varsigma_{, u} h_{, x x} & \varsigma h_{, x x x}
\end{array}\right]
$$

where $\varsigma \equiv\left(h_{, x x u} / h_{, x x}\right)_{, u}-\left(h_{, x x} / 2\right)\left(h_{, x} / h_{, x x}\right)_{, x}$. The obstruction elements for $\operatorname{rank} \boldsymbol{R}_{[(21)]}^{\# 2}=0$ are therefore given by

$$
\left(\frac{h_{, x x x}}{h_{, x x}}\right)_{, x}, \quad\left(\frac{h_{, x x x}}{h_{, x x}}\right)_{, u},
$$

and collaterally

$$
\left(\frac{h_{, x x u}}{h_{, x x}}\right)_{, u}-\frac{h_{, x x}}{2}\left(\frac{h_{, x}}{h_{, x x}}\right)_{, x} .
$$

If these criteria (5.21) are all vanishing, $\operatorname{rank} \boldsymbol{R}_{[(21)]}^{\# 2}=0$ and we can parameterise $h$ by a nonzero function $h_{1}$ as

$$
h(u, x)=h_{0}(u)+\mathrm{e}^{c_{1}\left(x+h_{1}(u)\right)}-2 x h_{1, u u}(u)
$$


where $h_{0}$ is an arbitrary functions of $u$ and $c_{1}$ is a nonzero constant, thereby allowing us to obtain $3 \mathrm{KVs}$

$$
\begin{aligned}
K_{1}= & \partial_{v} \\
K_{2}= & \partial_{u}-\frac{1}{2}\left(h_{0}+h_{1, u}^{2}-2 x h_{1, u u}\right) \partial_{v}-h_{1, u} \partial_{x}, \\
K_{3}= & u \partial_{u}-\left(v+\frac{u h_{0}+\int \mathrm{d} u h_{0}}{2}-u x h_{1, u u}+\frac{u h_{1, u}^{2}-\int \mathrm{d} u h_{1, u}^{2}}{2}\right. \\
& \left.+\left(\frac{2}{c_{1}}-x\right) h_{1, u}\right) \partial_{v}-\left(\frac{2}{c_{1}}+u h_{1, u}\right) \partial_{x},
\end{aligned}
$$

together with their commutators

$$
\left[K_{1}, K_{2}\right]=0, \quad\left[K_{2}, K_{3}\right]=K_{2}, \quad\left[K_{3}, K_{1}\right]=K_{1}
$$

Let us next consider the case in which $\operatorname{rank} \boldsymbol{R}_{[(21)]}^{\# 2} \neq 0$. Since $\operatorname{rank} \boldsymbol{R}_{[(21)]}^{\# 2}=2$ implies that there exists a single $\mathrm{KV} \partial_{v}$, we shall concentrate on the case $\operatorname{rank} \boldsymbol{R}_{[(21)]}^{\# 2}=1$. As a result, the conditions for which the metric (5.6) admits $2 \mathrm{KVs}$ are identified as shown in Figure 5b. This will be achieved by separating our analysis into the four types (A, B, C, D) based on the nonzeroness of eq. (5.21). We shall fix the explicit forms of $h(u, x)$, the 2 nd $\mathrm{KV}$ and its commutator in the rest of this subsection.

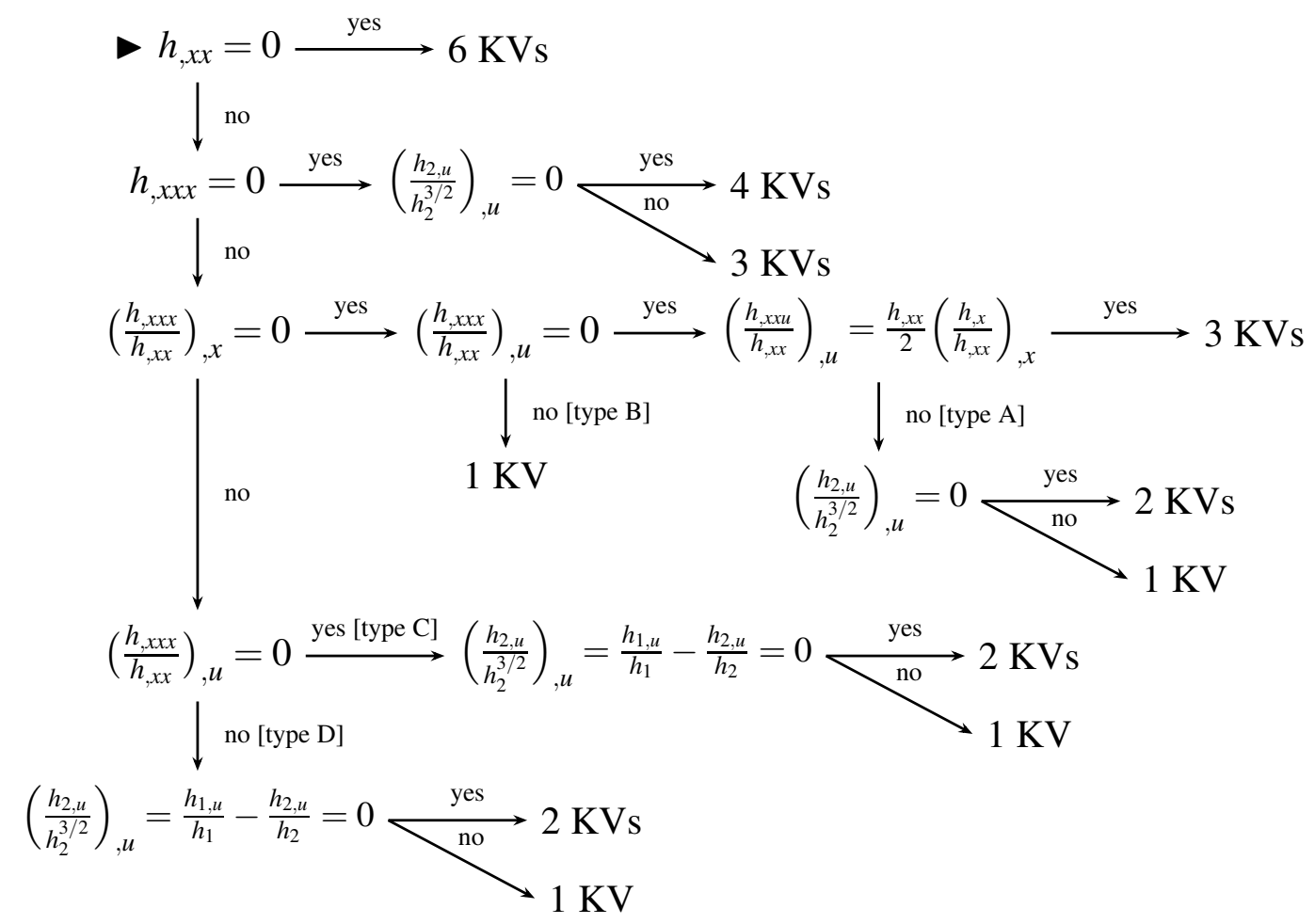

Figure 5b. A flowchart to classify the number of KVs of the pp-wave spacetime (5.6) in 3D. 
Type A: In this type, the function $h$ solves the PDEs $\left(h_{, x x x} / h_{, x x}\right)_{, x}=0$ and $\left(h_{, x x x} / h_{, x x}\right)_{, u}=0$ simultaneously, whereas $\varsigma=\left(h_{, x x u} / h_{, x x}\right)_{, u}-\left(h_{, x x} / 2\right)\left(h_{, x} / h_{, x x}\right)_{, x} \neq 0$. Thus we have

$$
h(u, x)=h_{0}(u)+\mathrm{e}^{c_{1}\left(x+h_{1}(u)\right)}-2 x\left(h_{1, u u}(u)-h_{2}(u)\right)
$$

where $\left\{h_{0}, h_{1}, h_{2}\right\}$ are functions of $u$ and $c_{1}$ is a constant. $h_{2}$ and $c_{1}$ are nonvanishing. From eq. (5.20), $\boldsymbol{R}_{[(21)]}^{\# 2} \boldsymbol{\omega}=0$ imposes

$$
\omega_{e}=\gamma \omega_{v}, \quad \gamma \equiv-\frac{\varsigma h_{, x x u}-\varsigma_{, u} h_{, x x}}{\varsigma h_{, x x x}}=\frac{h_{2, u}-c_{1} h_{2} h_{1, u}}{c_{1} h_{2}} .
$$

In a nod to eq. (5.28) and the basis (5.10), we take an orthonormal basis $\left\{e_{1}^{a}, e_{2}^{a}, e_{3}^{a}\right\}$ as

$$
\begin{array}{llrl}
e_{1}^{a} \equiv-\gamma u^{a}+e^{a}, & e_{2}^{a} \equiv \gamma^{-1} v^{a}+e^{a}, & e_{3}^{a} \equiv \gamma u^{a}-\gamma^{-1} v^{a}-e^{a}, & \text { for } \gamma \neq 0, \\
e_{1}^{a} \equiv e^{a}, & e_{2}^{a} \equiv \frac{1}{\sqrt{2}}\left(u^{a}+v^{a}\right), & e_{3}^{a} \equiv \frac{1}{\sqrt{2}}\left(u^{a}-v^{a}\right), & \text { for } \gamma=0,
\end{array}
$$

whence any $\mathrm{KV}$ is reduced to the form $K^{a}=\omega_{2} e_{2}^{a}+\omega_{3} e_{3}^{a}$. In either case, the basis satisfies $g^{a b}=e_{1}^{a} e_{1}^{b}+e_{2}^{a} e_{2}^{b}-e_{3}^{a} e_{3}^{b}$ and $\tau_{2}=\tau_{3}$ with $\tau_{2} \neq 0$. Therefore from Figure $3 \mathrm{~b}$ rank $\boldsymbol{R}_{\text {cls. } 2}^{l \# 2}$ determines the existence of the $2 \mathrm{nd} \mathrm{KV}$.

It is simple to see that the third, fourth and fifth rows of $\boldsymbol{R}_{\mathrm{cls} .2}^{\mathrm{\# 2}}$ are left nonvanishing, yielding the condition to have $\operatorname{rank} \boldsymbol{R}_{\mathrm{cls} .2}^{l \# 2}=0$ as $\left(h_{2, u} / h_{2}^{3 / 2}\right)_{, u}=0$. The solution to the ODE categorises into $h_{2}=$ const. or $h_{2} \propto u^{-2}$. For $h_{2}=c_{2}=$ const., the 2 nd KV arises in the form

$$
K_{2}=\partial_{u}-\frac{1}{2}\left(h_{0}+h_{1, u}^{2}-2 x h_{1, u u}-2 c_{2} h_{1}\right) \partial_{v}-h_{1, u} \partial_{x}
$$

with the commutator $\left[K_{1}, K_{2}\right]=0$. For $h_{2}=c_{2} u^{-2}\left(c_{2}=\right.$ const. $\left.\neq 0\right)$, the 2 nd KV reads

$$
\begin{aligned}
K_{2}= & u \partial_{u}-\left(v+\frac{u h_{0}+\int \mathrm{d} u h_{0}}{2}-u x h_{1, u u}+\frac{u h_{1, u}^{2}-\int \mathrm{d} u h_{1, u}^{2}}{2}\right. \\
& \left.+\left(\frac{2}{c_{1}}-x\right) h_{1, u}-c_{2} \int \frac{\mathrm{d} u}{u^{2}}\left(\frac{2}{c_{1}}+u h_{1, u}\right)\right) \partial_{v}-\left(\frac{2}{c_{1}}+u h_{1, u}\right) \partial_{x}
\end{aligned}
$$

with the commutator $\left[K_{1}, K_{2}\right]=-K_{1}$.

Type B: Here the function $h$ is characterised by the two conditions $\left(h_{, x x x} / h_{, x x}\right)_{, x}=0$ and $\left.\overline{(h, x x x} / h_{, x x}\right)_{, u} \neq 0$, leading to the form

$$
h(u, x)=h_{0}(u)+x h_{1}(u)+\mathrm{e}^{h_{2}(u)+x h_{3}(u)},
$$

where $\left\{h_{0}, h_{1}, h_{2}, h_{3}\right\}$ are arbitrary functions of $u$, but $h_{3, u}(u) \neq 0$ has to be true for the latter condition. It is required by $\boldsymbol{R}_{[(21)]}^{\# 2} \boldsymbol{\omega}=0$ that

$$
h_{3, u} \omega_{v}=0, \quad h_{3, u} \omega_{e}=0,
$$

concluding that $\omega_{v}=\omega_{e}=0$, so there is no possibility of finding the $2 \mathrm{nd} \mathrm{KV}$. 
Type $C$ : Since the function $h$ is the general solution to $\left(h_{, x x x} / h_{, x x}\right)_{, u}=0$, we have

$$
h(u, x)=h_{0}(u)+x h_{1}(u)+h_{2}(u) h_{3}(x),
$$

where $\left\{h_{0}, h_{1}\right\}$ are arbitrary functions of $u$, and $\left\{h_{2}, h_{3}\right\}$ are respectively nonzero functions of $u$ and of $x$. As $\left(h_{, x x x} / h_{, x x}\right)_{, x}=0$ is satisfied nowhere, it is stipulated that $\left(h_{3, x x x} / h_{3, x x}\right)_{, x} \neq 0$. From this and the first row of $\boldsymbol{R}_{[(21)]}^{\# 2}$, it is inevitable that $\omega_{e}=0$. The leftover components of $\boldsymbol{R}_{[(21)]}^{\# 2}$ put the requirements to have $\operatorname{rank} \boldsymbol{R}_{[(21)]}^{\# 2}=1$ as

$$
\left(h_{2, u} / h_{2}\right)_{, u}=0, \quad h_{1, u} / h_{1}=h_{2, u} / h_{2} .
$$

Assuming eqs. (5.35) and using new basis $\left\{e_{1}^{a}, e_{2}^{a}, e_{3}^{a}\right\}$ defined by eq. (5.30), it is easy to see that $\operatorname{rank} \boldsymbol{R}_{\mathrm{cls} .2}^{\Perp \# 2}=0$. For $h_{1}=c_{1}=$ const. and $h_{2}=c_{2}=$ const., the 2 nd KVs is given by

$$
K_{2}=\partial_{u}-\frac{h_{0}}{2} \partial_{v}
$$

with the commutator $\left[K_{1}, K_{2}\right]=0$. For $h_{1}=c_{1} u^{-2}\left(c_{1}=\right.$ const.) and $h_{2}=c_{2} u^{-2}\left(c_{2}=\right.$ const.), the $2 \mathrm{nd} \mathrm{KVs}$ is expressed as

$$
K_{2}=u \partial_{u}-\frac{1}{2}\left(u h_{0}+\int \mathrm{d} u h_{0}+2 v\right) \partial_{v}
$$

with the commutator $\left[K_{1}, K_{2}\right]=-K_{1}$.

Type D: It is immediately seen from the first row of $\boldsymbol{R}_{[(21)]}^{\# 2}$ that

$$
\omega_{e}=-\frac{\sigma_{, u}}{\sigma_{, x}} \omega_{v}, \quad \sigma(u, x) \equiv \frac{h_{, x x x}}{2 h_{, x x}}
$$

where the valuable $\sigma(u, x)$ inherits from the definition (4.34). The remaining entries do not have illuminating expressions to be described here. Leaving aside the full implications of $\boldsymbol{R}_{[(21)]}^{\# 2}$, we proceed to the analysis of class 2 to simplify the reasoning. By choosing an orthonormal basis $\left\{e_{1}^{a}, e_{2}^{a}, e_{3}^{a}\right\}$ as

$$
e_{1}^{a} \equiv \frac{\sigma_{, u}}{\sigma_{, x}} u^{a}+e^{a}, \quad e_{2}^{a} \equiv-\frac{\sigma_{, x}}{\sigma_{, u}} v^{a}+e^{a}, \quad e_{3}^{a} \equiv-\frac{\sigma_{, u}}{\sigma_{, x}} u^{a}+\frac{\sigma_{, x}}{\sigma_{, u}} v^{a}-e^{a},
$$

we have

$$
\kappa_{1}=-\eta_{1}=\frac{\sigma_{, x x}}{\sigma_{, x}}-\frac{\sigma_{, u x}}{\sigma_{, u}}
$$

From the result of subsection 3.1.1, eq. (5.39) has to be zero so that the 2nd KV can exist. Solving a PDE $\sigma_{, x x} / \sigma_{, x}-\sigma_{, u x} / \sigma_{, u}=0$, we obtain

$$
\sigma(u, x)=\sigma\left(x+h_{4}(u)\right)
$$


where $h_{4}$ is an arbitrary function of $u$. Subsequently, the definition of $\sigma$ (5.37) gives

$$
h(u, x)=h_{0}(u)+x\left(h_{1}(u)-2 h_{4, u u}(u)\right)+h_{2}(u) h_{3}\left(x+h_{4}(u)\right),
$$

where $\left\{h_{0}, h_{1}, h_{2}, h_{3}\right\}$ are arbitrary functions of one variable such that $\left(h_{3, x x x} / h_{3, x x}\right)_{, x} \neq 0$ and $h_{4, u} \neq 0$. Note that the existence of the $2 \mathrm{nd} \mathrm{KV}$ is still not clear, so we go on to examining rank $\boldsymbol{R}_{\text {cls.2. }}^{l}$. It follows from the third and fourth rows of $\boldsymbol{R}_{\mathrm{cls} .2}^{l}$ that $\operatorname{rank} \boldsymbol{R}_{\mathrm{cls} .2}^{l} \# 2=0$ if $\left(h_{2, u} / h_{2}^{3 / 2}\right)_{, u}=0$ and $h_{1, u} / h_{1}=h_{2, u} / h_{2}$.

For $h_{1}=c_{1}=$ const. and $h_{2}=c_{2}=$ const., the 2 nd $\mathrm{KV}$ is given by

$$
K_{2}=\partial_{u}-\frac{1}{2}\left(h_{0}+h_{4, u}^{2}-2 x h_{4, u u}-c_{1} h_{4}\right) \partial_{v}-h_{4, u} \partial_{x},
$$

with the commutator $\left[K_{1}, K_{2}\right]=0$. For $h_{1}=c_{1} u^{-2}\left(c_{1}=\right.$ const.) and $h_{2}=c_{2} u^{-2}\left(c_{2}=\right.$ const.), the $2 \mathrm{nd} \mathrm{KVs}$ is expressed as

$$
\begin{aligned}
K_{2}= & u \partial_{u}-\frac{1}{2}\left(u h_{0}+\int \mathrm{d} u h_{0}+2 v-\int \mathrm{d} u h_{4, u}^{2}-2 x\left(u h_{4, u u}+h_{4, u}\right)+u h_{4, u}^{2}\right. \\
& \left.-c_{1}\left(\frac{h_{4}}{u}+\int \mathrm{d} u \frac{h_{4}}{u^{2}}\right)\right) \partial_{v}-u h_{4, u} \partial_{x},
\end{aligned}
$$

with the commutator $\left[K_{1}, K_{2}\right]=-K_{1}$.

\section{Conclusion}

The basic questions we addressed in this paper are whether there exists a set of invariants associated with the existence of KVs, and if so, how to construct it for a given Lorentzian manifold. Our contribution is to give affirmative answers to such questions in dimension 3 , extending the result for a Riemannian manifold [12]. According to our theorem in Section 1 , the number of linearly independent KVs can be counted using the algorithm described in Figure 1b, even if a given spacetime has a VSI property. As we have seen in Section 5, the theorem can classify a given spacetime into a hierarchy based on their level of symmetry.

It would be instructive to mention the algorithmic efficiency of the Cartan-Karlhede and our formulations. Given a Lorentzian manifold of dimension 3, the Cartan-Karlhede algorithm uses Cartan scalars and requires at most six differentiations of $R_{a b c}{ }^{d}$. In that case, one must assess the functionally independent 336 Cartan scalars, whence it reveals the number of KVs in principle. On the other hand, our algorithm uses the Ricci rotation coefficients, their derivatives and the ratio thereof. In the worst case, it may be implemented in line with Figure $6 \mathrm{a}$ and 66 differential invariants are required in total. In conjugation with this, our prescription requires up to the 3 rd derivatives of the curvature in $\boldsymbol{R}_{[(21)]}^{\# 2}$. Thus, our algorithm is more economic than that of the Cartan-Karlhede to count the number of KVs. The only price to pay for our method to work out is to solve the eigenvalue problem of the traceless Ricci operator $S^{a}{ }_{b}$ in the class 3. Fortunately, this is not a demanding task in dimension 3.

As an application, our theorem may enable us to derive the canonical form of metrics with a high degree of symmetry. In fact, the calculations we have carried out in Appendix B 


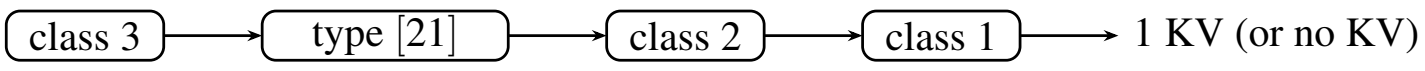

Figure 6a. A flow which requires the most differential invariants.

give the canonical form of metrics admitting $4 \mathrm{KVs}$, which is a reproduction and improvement of the classical result due to Kruchkovich [25]. As a side remark, we note that our algorithm does not ensure that local metrics endowed with a certain degree of symmetry exist in all branches of class 1-3. In other words, it seems that some values of rank $\boldsymbol{R}_{\mathrm{cls} . d}$ are prohibited in principle, resembling Fubini's theorem on the order of the isometry group. The classification of metrics with $3 \mathrm{KV}$ s makes this fact manifest. For instance, it can be shown that $\operatorname{rank} \boldsymbol{R}_{[(21)]}^{\# 1}$ cannot equal to zero. The detail of this extensive study will be reported in a forthcoming paper.

It is also noteworthy to comment that the local existence of $\mathrm{KVs}$ does not immediately give rise to the existence of the global isometry group due to topological restrictions. An emblematic sample is the black hole constructed by Bañados, Teitelboim and Zanelli (BTZ) [23]. The BTZ black hole solves the vacuum Einstein's equations with a negative cosmological constant and is obtained by the identification of points in AdS by the discrete isometry. From the curvature points of view, the BTZ metric obviously admits $6 \mathrm{KVs}$ generating $\mathfrak{s o}(2,2)$ algebra. In spite of this, only two of them are globally well-defined, since the rest of KVs is not single-valued under identifications [24]. It thus turns out that the global isometry group of the BTZ solution is broken from $\mathrm{SO}(2,2)$ down to $\mathrm{SO}(1,1) \times \mathrm{SO}(2)$.

Our algorithm can be extensible for a (semi-)Riemannian manifold $M$ of higher dimension. In this case, the Weyl tensor $W_{a b c}{ }^{d}$ also comes into play. In particular, the first obstruction matrix in $\operatorname{dimension} \operatorname{dim} M=n$ takes the form

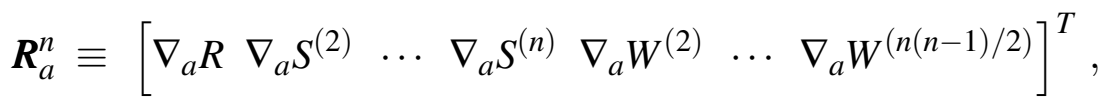

where $W^{(i)}$ are principal traces of the $i$-th powers of the Weyl operator $W^{a b}{ }_{c d}$, considered as an endomorphism of $\Lambda^{2} T(M)$. So the invariants associated with the existence of KVs can be specified based on $\boldsymbol{R}_{a}^{n}$. This line of extension would be a promising way to improve the past-proposed schemes. Further consideration of this shall be done elsewhere.

\section{Acknowledgement}

KT thanks Boris Kruglikov, Vladimir Matveev, Tohru Morimoto and Kazuhiro Shibuya for their advices and useful comments. KT is also grateful to Kazuhiro Shibuya, Yoshio Agaoka, Hiroshi Tamaru and other organisers of The Geometry Conference 2018 held in Hiroshima for their invitation. The work of MN is partially supported by Grant-in-Aid for Scientific Research from Ministry of Education, Science, Sports and Culture of Japan (16H03979 and 17H01091). 


\section{Appendix A. Relations amongst the Ricci rotation coefficients and their derivatives}

In this Appendix, we collect some relations amongst the Ricci rotation coefficients and their derivatives which are implicitly used in Sections 3 and 4.

Appendix A.1. For an orthonormal frame

Given an orthonormal frame $\left\{e_{i}^{a}, i=1,2,3\right\}$ satisfying

$$
g^{a b}=\imath e_{1}^{a} e_{1}^{b}+e_{2}^{a} e_{2}^{b}-\imath e_{3}^{a} e_{3}^{b},
$$

where $\imath=\operatorname{sgn}\left(g_{a b} e_{1}^{a} e_{1}^{b}\right)$ and its Ricci rotation coefficients (3.5), the following relations hold true.

\section{The commutation relations:}

$$
\begin{aligned}
& {\left[e_{1}, e_{2}\right]^{a}=-\imath \kappa_{1} e_{1}^{a}+\kappa_{2} e_{2}^{a}-\imath\left(\tau_{1}-\tau_{2}\right) e_{3}^{a},} \\
& {\left[e_{2}, e_{3}\right]^{a}=-\imath\left(\tau_{2}-\tau_{3}\right) e_{1}^{a}-\eta_{2} e_{2}^{a}-\imath \eta_{3} e_{3}^{a},} \\
& {\left[e_{3}, e_{1}\right]^{a}=\imath \eta_{1} e_{1}^{a}+\left(\tau_{1}+\tau_{3}\right) e_{2}^{a}+\imath \kappa_{3} e_{3}^{a} .}
\end{aligned}
$$

\section{The components of the Ricci tensor:}

$$
\begin{aligned}
R_{a b} e_{1}^{a} e_{1}^{b} & =\eta_{1}^{2}+\imath \eta_{1} \eta_{2}-\kappa_{3}^{2}+\imath \eta_{3} \kappa_{1}-\imath \kappa_{1}^{2}-\kappa_{2}^{2}+2 \imath \tau_{2} \tau_{3}-\imath £_{1} \kappa_{3}-\imath £_{3} \eta_{1}+£_{1} \kappa_{2}+£_{2} \kappa_{1} \\
R_{a b} e_{2}^{a} e_{2}^{b} & =-\eta_{3}^{2}+\eta_{1} \eta_{2}+\imath \eta_{2}^{2}-\kappa_{1}^{2}+\kappa_{2} \kappa_{3}-\imath \kappa_{2}^{2}-2 \tau_{3} \tau_{1}-\imath £_{3} \eta_{2}+\imath £_{1} \kappa_{2}-\imath £_{2} \eta_{3}+\imath £_{2} \kappa_{1} \\
R_{a b} e_{3}^{a} e_{3}^{b} & =\imath \eta_{3}^{2}-\eta_{1}^{2}-\eta_{2}^{2}+\kappa_{3}^{2}-\imath \eta_{3} \kappa_{1}-\imath \kappa_{3} \kappa_{2}-2 \imath \tau_{1} \tau_{2}+\imath £_{3} \eta_{1}+£_{3} \eta_{2}+\imath £_{1} \kappa_{3}+£_{2} \eta_{3} \\
R_{a b} e_{1}^{a} e_{2}^{b} & =-\eta_{3} \kappa_{3}-\imath \eta_{3} \kappa_{2}-\eta_{1} \tau_{3}+\imath \eta_{2} \tau_{3}+\eta_{1} \tau_{2}+\imath \eta_{2} \tau_{2}-\imath £_{3} \tau_{2}-\imath £_{2} \kappa_{3} \\
& =-\eta_{3} \kappa_{3}-\kappa_{3} \kappa_{1}-\eta_{1} \tau_{3}+\imath \eta_{2} \tau_{3}+\eta_{1} \tau_{1}+\imath \eta_{2} \tau_{1}-\imath £_{3} \tau_{1}-\imath £_{1} \eta_{3} \\
R_{a b} e_{2}^{a} e_{3}^{b} & =-\eta_{3} \eta_{1}-\eta_{1} \kappa_{1}-\kappa_{3} \tau_{3}+\imath \kappa_{2} \tau_{3}+\kappa_{3} \tau_{1}+\imath \kappa_{2} \tau_{1}+\imath £_{3} \kappa_{1}-\imath £_{1} \tau_{3} \\
& =-\eta_{1} \kappa_{1}+\imath \eta_{2} \kappa_{1}+\kappa_{3} \tau_{1}+\imath \kappa_{2} \tau_{1}-\kappa_{3} \tau_{2}+\imath \kappa_{2} \tau_{2}-\imath £_{1} \tau_{2}+\imath £_{2} \eta_{1} \\
R_{a b} e_{3}^{a} e_{1}^{b} & =-\imath \eta_{2} \kappa_{3}-\eta_{2} \kappa_{2}+\imath \eta_{3} \tau_{3}-\imath \kappa_{1} \tau_{3}+\imath \eta_{3} \tau_{2}+\imath \kappa_{1} \tau_{2}+£_{3} \kappa_{2}+£_{2} \tau_{3} \\
& =\imath \eta_{1} \kappa_{2}-\eta_{2} \kappa_{2}-\imath \eta_{3} \tau_{1}+\imath \kappa_{1} \tau_{1}+\imath \eta_{3} \tau_{2}+\imath \kappa_{1} \tau_{2}+£_{1} \eta_{2}-£_{2} \tau_{1}
\end{aligned}
$$

\section{Appendix A.2. For a double-null frame}

Given an orthonormal frame $\left\{u^{a}, v^{a}, e^{a}\right\}$ satisfying

$$
g^{a b}=u^{a} v^{b}+v^{a} u^{b}+e^{a} e^{b},
$$

and its Ricci rotation coefficients (3.18), the following relations hold true. 
The commutation relations:

$$
\begin{aligned}
& {[u, v]^{a}=\kappa_{v} u^{a}-\kappa_{u} v^{a}+\left(\tau_{u}-\tau_{v}\right) e^{a}} \\
& {[v, e]^{a}=-\eta_{v} u^{a}-\left(\tau_{v}-\tau_{e}\right) v^{a}+\eta_{e} e^{a},} \\
& {[e, u]^{a}=\left(\tau_{u}+\tau_{e}\right) u^{a}+\eta_{u} v^{a}-\kappa_{e} e^{a} .}
\end{aligned}
$$

\section{The components of the Ricci tensor:}

$$
\begin{aligned}
R_{a b} u^{a} u^{b} & =-\kappa_{e}^{2}-\kappa_{e} \kappa_{u}-2 \eta_{u} \tau_{e}-\eta_{u} \tau_{u}-\eta_{u} \tau_{v}+£_{e} \eta_{u}+£_{u} \kappa_{e}, \\
R_{a b} v^{a} v^{b} & =-\eta_{e}^{2}-\eta_{e} \kappa_{v}+2 \eta_{v} \tau_{e}-\eta_{v} \tau_{u}-\eta_{v} \tau_{v}+£_{e} \eta_{v}+£_{v} \eta_{e} \\
R_{a b} e^{a} e^{b} & =-2 \eta_{u} \eta_{v}-2 \eta_{e} \kappa_{e}+\eta_{e} \kappa_{u}+\kappa_{e} \kappa_{v}-\tau_{u}^{2}-\tau_{v}^{2}+£_{e} \tau_{u}+£_{e} \tau_{v}+£_{u} \eta_{e}+£_{v} \kappa_{e}, \\
R_{a b} u^{a} v^{b} & =-\eta_{e} \kappa_{e}+\kappa_{e} \kappa_{v}-2 \kappa_{u} \kappa_{v}-\tau_{e} \tau_{u}+\tau_{e} \tau_{v}-\tau_{u} \tau_{v}-\tau_{v}^{2}+£_{e} \tau_{v}-£_{u} \kappa_{v}+£_{v} \kappa_{e}-£_{v} \kappa_{u}, \\
& =-\eta_{e} \kappa_{e}+\eta_{e} \kappa_{u}-2 \kappa_{u} \kappa_{v}-\tau_{e} \tau_{u}-\tau_{u}^{2}+\tau_{e} \tau_{v}-\tau_{u} \tau_{v}+£_{e} \tau_{u}+£_{u} \eta_{e}-£_{u} \kappa_{v}-£_{v} \kappa_{u}, \\
R_{a b} v^{a} e^{b} & =-\eta_{v} \kappa_{e}-\eta_{v} \kappa_{u}+\eta_{e} \tau_{e}-\kappa_{v} \tau_{e}+\eta_{e} \tau_{v}+\kappa_{v} \tau_{v}-£_{e} \kappa_{v}-£_{v} \tau_{e}, \\
& =-2 \eta_{v} \kappa_{u}-\eta_{e} \tau_{u}+\eta_{e} \tau_{v}-£_{u} \eta_{v}+£_{v} \tau_{u}, \\
R_{a b} e^{a} u^{b} & =-\eta_{e} \eta_{u}-\eta_{u} \kappa_{v}-\kappa_{e} \tau_{e}+\kappa_{u} \tau_{e}+\kappa_{e} \tau_{u}+\kappa_{u} \tau_{u}-£_{e} \kappa_{u}+£_{u} \tau_{e}, \\
& =-2 \eta_{u} \kappa_{v}+\kappa_{e} \tau_{u}-\kappa_{e} \tau_{v}+£_{u} \tau_{v}-£_{v} \eta_{u} .
\end{aligned}
$$

\section{Appendix B. Canonical form of metrics admitting 4 Killing vectors}

Using the scheme developed in the present paper, we can obtain the canonical form of the metric admitting any number of KVs and the corresponding algebra. To make the discussion focused, we investigate in this appendix the case in which $4 \mathrm{KVs}$ exist. As described in section 4 , this occurs only for Segre types $[1,(11)],[(1,1), 1]$ and $[(21)]$. In each case, it turns out that we can actually obtain all the explicit metrics. Interestingly, these spacetimes are all homogeneous, in the sense that local isometry groups possess transitive actions on the manifold.

\section{Appendix B.1. Type $[1,(11)]$}

Let us begin with the case of Segre type $[1,(11)]$. Analysis in section 4.1 .1 reveals that $4 \mathrm{KVs}$ exist, provided

$$
\kappa_{2}=\tau_{2}+\tau_{3}=0, \quad \tau_{2}=\text { const. }
$$

together with eq. (4.7):

$$
\kappa_{1}=0, \quad \eta_{1}=0, \quad \kappa_{3}=-\kappa_{2} .
$$


Here $£_{1} \tau_{2}=0$ follows from $R_{23}=R_{32}$. With these spin connections, the first derivative of $\left\{e_{1}, e_{2}, e_{3}\right\}$ reads

$$
\begin{aligned}
& \nabla_{b} e_{1 a}=\tau_{2} e_{2 b} e_{3 a}-\tau_{2} e_{3 b} e_{2 a}, \\
& \nabla_{b} e_{2 a}=-\tau_{1} e_{1 b} e_{3 a}+\eta_{2} e_{2 b} e_{3 a}+e_{3 b}\left(-\tau_{2} e_{1 a}-\eta_{3} e_{3 a}\right), \\
& \nabla_{b} e_{3 a}=\tau_{1} e_{1 b} e_{2 a}+e_{2 b}\left(\tau_{2} e_{1 a}-\eta_{2} e_{2 a}\right)+\eta_{3} e_{3 b} e_{2 a} .
\end{aligned}
$$

It follows that $W_{a}=e_{2 a}+i e_{3 a}$ satisfies

$$
\nabla_{b} W_{a}=i\left(\tau_{1} e_{1 b}-\eta_{2} e_{2 b}+\eta_{3} e_{2 b}\right) W_{a}+i \tau_{2} e_{1 a} W_{b},
$$

hence

$$
\nabla_{(a} e_{1 b)}=0, \quad W_{[a} \nabla_{b} W_{c]}=0 .
$$

Then, there exist real functions $t, x, y$ and $\theta, \phi, f, \chi_{1}, \chi_{2}$ such that

$$
e_{1 a}=-f\left(\nabla_{a} t+\chi_{1} \nabla_{a} x+\chi_{2} \nabla_{a} y\right), \quad W_{a}=e^{i \theta+\phi}\left(\nabla_{a} x+i \nabla_{a} y\right) .
$$

By the redefinition $t \rightarrow \int f^{-1} \mathrm{~d} t$, one can set $f \equiv 1$ without loss of generality. Exploiting the $\mathrm{SO}(2)$ gauge freedom which rotates $\left(e_{2}, e_{3}\right), \theta=0$ is always achieved. The Killing equation $\nabla_{(a} e_{1 b)}=0$ then demands that the metric is independent of $t$. The condition $\tau_{2}$ (= const.) boils down to

$$
\partial_{y} \chi_{1}-\partial_{x} \chi_{2}=2 \tau_{2} e^{2 \phi}
$$

Using this relation, the trace-free part of Ricci tensor gives rise to Liouville's equation

$$
\left(\partial_{x}^{2}+\partial_{y}^{2}\right) \phi=-k e^{2 \phi}, \quad k \equiv-\frac{1}{2}\left(3 \lambda_{1}+8 \tau_{2}^{2}\right) .
$$

It follows that $\mathrm{d} \Sigma_{k}^{2}=e^{2 \phi}\left(\mathrm{d} x^{2}+\mathrm{d} y^{2}\right)$ corresponds to the space $\Sigma_{k}$ with a constant sectional curvature $k$, which can be normalised to be 0 or \pm 1 , and the scalar curvature is given by $R=2\left(k+\tau_{2}^{2}\right)$. The local solution to Liouville's equation can be chosen to be

$$
\phi=-\log \left(1+\frac{k}{4}\left(x^{2}+y^{2}\right)\right), \quad \chi=\frac{\tau_{2}}{1+\frac{k}{4}\left(x^{2}+y^{2}\right)}(y \mathrm{~d} x-x \mathrm{~d} y),
$$

where $\chi=\chi_{1} \mathrm{~d} x+\chi_{2} \mathrm{~d} y$. Defining $x+i y=\frac{2}{\sqrt{k}} \tan \left(\frac{\sqrt{k}}{2} \theta\right) e^{i \phi}$, we therefore arrive at

$$
\mathrm{d} s^{2}=-\left[\mathrm{d} t-4 \tau_{2}\left(\frac{\sin \left(\frac{\sqrt{k}}{2} \theta\right)}{\sqrt{k}}\right) \mathrm{d} \phi\right]^{2}+\mathrm{d} \theta^{2}+\left(\frac{\sin (\sqrt{k} \theta)}{\sqrt{k}}\right)^{2} \mathrm{~d} \phi^{2}
$$


If $k=-4 \tau_{2}^{2}<0$, we have $\mathrm{AdS}_{3}$ for which the number of $\mathrm{KVs}$ is enhanced to 6. Otherwise, we have precisely $4 \mathrm{KVs}$

$$
\begin{aligned}
& K_{1}=\partial_{t} \\
& K_{2}=\frac{2 \tau_{2}}{\sqrt{k}} \cos \phi \tan \left(\frac{\sqrt{k}}{2} \theta\right) \partial_{t}-\sin \phi \partial_{\theta}-\frac{\sqrt{k}}{2} \cos \phi\left[\cot ^{2}\left(\frac{\sqrt{k}}{2} \theta\right)-1\right] \tan \left(\frac{\sqrt{k}}{2} \theta\right) \partial_{\phi}, \\
& K_{3}=\frac{2 \tau_{2}}{\sqrt{k}} \sin \phi \tan \left(\frac{\sqrt{k}}{2} \theta\right) \partial_{t}+\cos \phi \partial_{\theta}-\frac{\sqrt{k}}{2} \sin \phi\left[\cot ^{2}\left(\frac{\sqrt{k}}{2} \theta\right)-1\right] \tan \left(\frac{\sqrt{k}}{2} \theta\right) \partial_{\phi}, \\
& K_{4}=\partial_{\phi}
\end{aligned}
$$

satisfying

$$
\left[K_{2}, K_{3}\right]=-\left(2 \tau_{2} K_{1}+k K_{4}\right), \quad\left[K_{2}, K_{4}\right]=K_{3}, \quad\left[K_{3}, K_{4}\right]=-K_{2} .
$$

For $\tau_{2}=0$, the metric collapses to $\mathbb{R} \times \Sigma_{k}$, which is locally symmetric. For $\tau_{2}\left(k+4 \tau_{2}^{2}\right) \neq 0$ with $k=-1$, the metric describes the 3-dimensional Gödel universe, which is sourced by a dust with a negative cosmological constant (see e.g. [26]).

\section{Appendix B.2. Type $[(1,1) 1]$}

In this case, we have $\kappa_{1}=\kappa_{2}=\eta_{2}=\eta_{3}=0$ and $\tau_{1}=\tau_{3}=$ const., for which $e_{2 a}$ is Killing and $W_{a}^{ \pm}=e_{1 a} \pm e_{3 a}$ are hypersurface-orthogonal. Since the rest of the derivation is parallel to the $[1,(11)]$ case, we only show the final results:

$$
\mathrm{d} s^{2}=\left[\mathrm{d} t+4 \tau_{1}\left(\frac{\sin \left(\frac{\sqrt{k}}{2} \theta\right)}{\sqrt{k}}\right) \mathrm{d} \phi\right]^{2}+\mathrm{d} \theta^{2}-\left(\frac{\sin (\sqrt{k} \theta)}{\sqrt{k}}\right)^{2} \mathrm{~d} \phi^{2},
$$

where $\tau_{1}$ is a constant. This is the double Wick-rotated version of eq. (B.10).

Appendix B.3. Type $[(2,1)]$

A class of metrics with $4 \mathrm{KVs}$ exists also for the type $[(2,1)]$, for which

$$
\kappa_{e}=0, \quad \eta_{u}=0, \quad \sigma=0 .
$$

The second obstruction matrix $\boldsymbol{R}_{[(21)]}$ given by eq. (4.35) must vanish identically, yielding

$$
\tau_{v}=\text { const. } \quad \Sigma=\text { const. },
$$

where $£_{u} \tau_{v}=0$ follows from $S_{a b} e^{a} u^{b}=0$. These are exhaustive information supplied from the condition for $4 \mathrm{KVs}$ in type $[(2,1)]$.

The definition of $\Sigma, \sigma=0$ and Bianchi identity are combined to give 1st-order system for $\varphi=\frac{1}{2} \log \left(S_{v v}\right)$ as

$$
£_{u} \varphi=-\kappa_{u}, \quad £_{v} \varphi=\kappa_{v}-e^{\varphi} \Sigma, \quad £_{e} \varphi=-\tau_{e}-\tau_{v} .
$$


The compatibility conditions eq. (A.5) for these equations give

$$
2 e^{\varphi} \Sigma \tau_{v}=0
$$

which branches into (i) $\tau_{v}=0$ and (ii) $\Sigma=0$.

Before proceeding, let us note that the Segre type $[(2,1)]$ allows the following gauge freedom for the choice of null basis $\left\{u_{a}, v_{a}, e_{a}\right\}$ :

$$
u_{a} \rightarrow a u_{a}, \quad v_{a} \rightarrow a^{-1} v_{a}, \quad e_{a} \rightarrow e_{a},
$$

and

$$
u_{a} \rightarrow u_{a}, \quad v_{a} \rightarrow v_{a}-\frac{1}{2} b^{2} u_{a}+b e_{a}, \quad e_{a} \rightarrow e_{a}-b u_{a}
$$

where $a$ and $b$ are arbitrary functions. By these transformations, $\tau_{v} \equiv e^{a} v^{b} \nabla_{b} u_{a}$ and $\Sigma \equiv$ $£_{v}\left(e^{-\varphi}\right)+\kappa_{v} e^{-\varphi}$ remain invariant. In contrast, $\tau_{u} \equiv-v^{a} u^{b} \nabla_{b} e_{a}$ and $\tau_{e} \equiv v^{a} e^{b} \nabla_{b} u_{a}$ vary as $\tau_{u} \rightarrow \tau_{u}$ and $\tau_{e} \rightarrow \tau_{e}+e^{b} \nabla_{b} \log a$ under eq. (B.18), which permits us to set $\tau_{e}+\tau_{u}=0$. Since $[u, e]^{a}=0$ is now satisfied because the condition $\kappa_{e}=\eta_{u}=0$ does not change under eq. (B.18), one can introduce local coordinates $(x, y, z)$ in such a way that $u^{a}$ and $e^{a}$ form the coordinate vectors

$$
u^{a}=\left(\partial_{y}\right)^{a}, \quad v^{a}=V_{1}\left(\partial_{x}\right)^{a}+V_{2}\left(\partial_{y}\right)^{a}+V_{3}\left(\partial_{z}\right)^{a}, \quad e^{a}=\left(\partial_{z}\right)^{a},
$$

where $V_{i}=V_{i}(x, y, z)$. Lowering indices, we have

$$
u_{a}=\frac{\nabla_{a} x}{V_{1}}, \quad v_{a}=\nabla_{a} y-\frac{V_{2}}{V_{1}} \nabla_{a} x, \quad e_{a}=\nabla_{a} z-\frac{V_{3}}{V_{1}} \nabla_{a} x .
$$

The hypersurface orthogonality of $u_{a}$ is a direct consequence of $\eta_{u}=0$ and $\kappa_{e}=0$. In this basis, $\tau_{v}$ is computed to be

$$
\tau_{v}=-\frac{1}{2} V_{1}\left[\partial_{y}\left(\frac{V_{3}}{V_{1}}\right)+\partial_{z}\left(\frac{1}{V_{1}}\right)\right]
$$

The following discussion will be divided according to $\tau_{v}=0$ or $\Sigma=0$.

Appendix B.3.1. $\tau_{v}=0$ case Setting $\tau_{v}=0$ in eq. (B.22), one finds a local function $F=F(x, y, z)$ satisfying

$$
\frac{V_{3}}{V_{1}}=-\partial_{z} F, \quad \frac{1}{V_{1}}=\partial_{y} F
$$

Inserting this into $S_{a b} e^{a}=0$, one finds a function $f_{1}=f_{1}(x)$ such that

$$
\partial_{y} V_{2} \partial_{y} F+2 V_{2} \partial_{y}^{2} F+\frac{\partial_{x} \partial_{y} F-\partial_{z} F \partial_{y} \partial_{z} F}{\partial_{y} F}=f_{1},
$$


which is further integrated to give

$$
V_{2}=-\frac{1}{\left(\partial_{y} F\right)^{2}}\left[\partial_{x} F-\frac{1}{2}\left(\partial_{z} F\right)^{2}-f_{1}(x) F+\frac{1}{2} f_{2}(x, z)\right]
$$

where $f_{2}=f_{2}(x, z)$ is an arbitrary function of $x$ and $z$. Inspecting eqs. (B.23) and (B.25), one obtains

$$
R=0, \quad \varphi=\frac{1}{2} \log \left(-\frac{\partial_{z}^{2} f_{2}}{2\left(\partial_{y} F\right)^{2}}\right)
$$

Substitution of this expression of $\varphi$ into $\sigma \equiv £_{e} \varphi+\tau_{v}+\tau_{e}=0$, one gets $\partial_{z}^{3} f_{2}(x, z)=0$. Upon integration, we find

$$
f_{2}(x, z)=f_{20}(x)+f_{21}(x) z+f_{22}(x) z^{2} .
$$

The condition $S_{v v} \neq 0$ asks for $f_{22}(x) \neq 0 . \Sigma \equiv f_{v}\left(e^{-\varphi}\right)+\kappa_{v} e^{-\varphi}$ is now computed to

$$
\Sigma=\frac{-2 f_{1}(x) f_{22}(x)+f_{22}^{\prime}(x)}{2\left(-f_{22}(x)\right)^{3 / 2}}
$$

We have all ingredients in place to obtain the explicit metric form. Defining $\tilde{y}=F(x, y, z)$, the metric becomes

$$
\mathrm{d} s^{2}=2 \mathrm{~d} \tilde{y} \mathrm{~d} x+\mathrm{d} z^{2}+\mathrm{d} x^{2}\left[f_{20}(x)+f_{21}(x) z+f_{22}(x) z^{2}+\tilde{y} f_{1}(x)\right]
$$

Further change of variable $x=h(\hat{x}), \tilde{y}=\hat{y} / h^{\prime}(\hat{x})$ renders the metric into

$$
\mathrm{d} s^{2}=2 \mathrm{~d} \hat{x} \mathrm{~d} \hat{y}+\mathrm{d} z^{2}+\mathrm{d} \hat{x}^{2}\left[\hat{f}_{20}+\hat{f}_{21} z+\hat{f}_{22} z^{2}+\frac{\hat{y}}{h^{\prime}(\hat{x})}\left(\hat{f}_{1}+h^{\prime \prime}(\hat{x})\right)\right],
$$

where $\hat{f}_{1}(\hat{x})=h^{\prime}(\hat{x})^{2} f_{1}(h(\hat{x}))$ and $\hat{f}_{2 i}(\hat{x})=h^{\prime}(\hat{x})^{2} f_{2 i}(h(\hat{x}))(i=0,1,2)$. By choosing $h_{1}^{\prime \prime}(\hat{x})=$ $-\hat{f}_{1}(\hat{x})$ and omitting hats, one obtains the metric of the following form

$$
\mathrm{d} s^{2}=2 \mathrm{~d} x \mathrm{~d} y+\mathrm{d} z^{2}+\mathrm{d} x^{2}\left[f_{20}(x)+f_{21}(x) z+f_{22}(x) z^{2}\right] .
$$

This amounts to setting $f_{1}(x)=0$ in the metric (B.29). Thus, equation (B.28) is integrated to give

$$
f_{22}(x)=-\frac{1}{\left(\Sigma x-c_{1}\right)^{2}}
$$

where $c_{1}$ and $\Sigma$ are constants. This metric describes the pp-wave, whose $4 \mathrm{KVs}$ were already obtained in section 5.2. The special case $\Sigma=0$ corresponds to the locally symmetric space which admits a covariantly constant Ricci tensor $\nabla_{a} R_{b c}=0$. 
Appendix B.3.2. $\Sigma=0$ case We assume $\tau_{v} \neq 0$ henceforth, since the metric (B.31) with $\Sigma=0$ in eq. (B.32) is recovered for the $\tau_{v}=0$ case. Equation (B.22) is solved as

$$
\partial_{y} V_{3}=-2 \tau_{v}+\frac{\partial_{z} V_{1}+V_{3} \partial_{y} V_{1}}{V_{1}}
$$

Inserting this into $S_{a b} e^{b}=0$, one finds a function $k_{1}(x)$ satisfying

$$
\partial_{y} V_{2}=-k_{1} V_{1}+V_{3}\left(-2 \tau_{v}+\frac{\partial_{z} V_{1}}{V_{1}}\right)+\frac{2 V_{2} \partial_{y} V_{1}}{V_{1}}+\partial_{x} V_{1}
$$

It follows that the scalar curvature is a negative constant $R=-6 \tau_{v}^{2}<0$. The first and third conditions in eq. (B.16) give rise to

$$
\varphi=-2 \tau_{v} z+\log \left(k_{2}(x) V_{2}\right)
$$

where $k_{2}=k_{2}(x)$ is an arbitrary function. Setting $\Sigma=0$ in the second condition of eq. (B.16), $k_{1}$ is subjected to

$$
k_{1}(x)=-\frac{k_{2}^{\prime}(x)}{k_{2}(x)}
$$

Comparison of $S_{v v}=e^{2 \varphi}$ in a coordinate basis with the one given by eq. (B.35) assures the existence of a function $k_{3}=k_{3}(x, y)$ such that

$$
\begin{aligned}
k_{3}(x, y)= & -\frac{\partial_{x} V_{3}}{V_{1}}+\frac{-2 V_{2}+V_{3}^{2}}{V_{1}^{3}} \partial_{z} V_{1}-\frac{V_{3}}{V_{1}^{2}} \partial_{z} V_{3}+\frac{V_{3}}{V_{1}} \partial_{x} V_{1}+\frac{\partial_{z} V_{2}}{V_{1}^{2}} \\
& +\frac{V_{3}}{V_{1}^{2}}\left(-k_{1} V_{1}-\tau_{v} V_{3}\right)+\frac{2 \tau_{v} V_{2}}{V_{1}^{2}}+\frac{e^{-4 \tau_{v} z}}{4 \tau_{v}} k_{2}^{2}
\end{aligned}
$$

which is arranged into

$$
\partial_{x}\left[-\frac{V_{3} e^{2 \tau_{v} z}}{V_{1} k_{2}}\right]=\partial_{z}\left[\frac{e^{2 \tau_{v} z}}{2 k_{2}}\left(\frac{-2 V_{2}+V_{3}^{2}}{V_{1}^{2}}+\frac{k_{3}}{\tau_{v}}+\frac{e^{-4 \tau_{v} z}}{4 \tau_{v}^{2}} k_{2}^{2}\right)\right] .
$$

This implies the existence of a function $F_{1}=F_{1}(x, y, z)$ such that the terms in the square bracket on the left-hand side is $\partial_{z} F_{1}$ and the terms in the square bracket on the left-hand side is $\partial_{x} F_{1}$. This condition is simplified to

$$
\begin{aligned}
V_{2} & =\frac{e^{-4 \tau_{v} z}}{8 \tau_{v}^{2}} V_{1}^{2}\left[k_{2}^{2}+4 e^{4 \tau_{v} z} \tau_{v} k_{3}+4 \tau_{v}^{2} k_{2}^{2}\left(\partial_{z} F_{1}\right)^{2}-8 e^{2 \tau_{v} z} \tau_{v}^{2} k_{2} \partial_{x} F_{1}\right] \\
V_{3} & =-e^{-2 \tau_{v} z} k_{2} V_{1} \partial_{z} F_{1}
\end{aligned}
$$

The compatibility of eqs. (B.33) and (B.39) gives

$$
V_{1}=-\frac{e^{2 \tau_{v} z}}{k_{4}(x, y)-k_{2}(x) \partial_{y} F_{1}(x, y, z)}
$$


where $k_{4}(x, y)$ is a function independent of $z$. Together with eq. (B.40), the compatibility of eqs. (B.34) and (B.39) gives

$$
k_{3}=k_{3}(x), \quad k_{4}(x, y)=k_{2}(x) k_{41}(y) .
$$

We have exhausted the constrains coming from the vanishing of second obstruction matrix in class 3 of Segre [(21)].

Let us now move on to obtaining the metric form. Defining $\tilde{x}=\int k_{2}(x) \mathrm{d} x, \tilde{y}=$ $F_{1}(x, y, z)-\int k_{41}(y) \mathrm{d} y$, we get

$$
\mathrm{d} s^{2}=2 e^{-2 \tau_{v} z} \mathrm{~d} \tilde{x} \mathrm{~d} \tilde{y}+\mathrm{d} z^{2}+\mathrm{d} \tilde{x}^{2}\left(-\frac{e^{-4 \tau_{v} z}}{4 \tau_{v}^{2}}-\frac{\tilde{k}(\tilde{x})}{\tau_{v}}\right)
$$

where $\tilde{k}(\tilde{x})=k_{31}(x) / k_{2}(x)^{2}$. We change coordinates further to

$$
\hat{x}=\int e^{-2 \tau_{v} h(\tilde{x})} \mathrm{d} \tilde{x}, \quad \tilde{y}=\hat{y}-\frac{1}{2 \tau_{v}} e^{2 \tau_{v}(\hat{z}+h(\tilde{x}))} h^{\prime}(\tilde{x}), \quad z=\hat{z}+h(\tilde{x}),
$$

and choose $h(\tilde{x})$ to satisfy $\tilde{k}(\tilde{x})+\tau_{v} h^{\prime}(\tilde{x})^{2}+h^{\prime \prime}(\tilde{x})=0$. Then, the resulting metric is eq. (B.42) replaced by $(\tilde{x}, \tilde{y}, z) \rightarrow(\hat{x}, \hat{y}, \hat{z})$ and a vanishing $\tilde{k}(\tilde{x})$. Namely, we obtain

$$
\mathrm{d} s^{2}=2 e^{-2 \tau_{v} z} \mathrm{~d} x \mathrm{~d} y+\mathrm{d} z^{2}-\frac{1}{4 \tau_{v}^{2}} e^{-4 \tau_{v} z} \mathrm{~d} x^{2},
$$

where we have dropped hats from the variables. This metric represents the plane-wave (or equivalently the Kundt class), but not the pp-wave. The KVs are given by

$$
K_{1}=\partial_{x}, \quad K_{2}=\partial_{y}, \quad K_{3}=y \partial_{y}+\frac{1}{2 \tau_{v}} \partial_{z}, \quad K_{4}=-\frac{e^{2 \tau_{v} z}}{2 \tau_{v}^{2}} \partial_{x}+y^{2} \partial_{y}+\frac{y}{\tau_{v}} \partial_{z},
$$

whose nonvanishing commutation relations are

$$
\left[K_{2}, K_{3}\right]=K_{2}, \quad\left[K_{2}, K_{4}\right]=2 K_{3}, \quad\left[K_{3}, K_{4}\right]=K_{4} .
$$

This subalgebra is $\mathfrak{s l}(2, \mathbb{R})$. One can easily find that the above metric (B.44) recovers eq. (B.47d) below.

Appendix B.3.3. Remarks We conclude that the Segre [(21)] allows two metrics (B.31) and (B.44) with $4 \mathrm{KVs}$. Let us compare our results with those in the literature. The classification of spacetimes admitting $4 \mathrm{KVs}$ for Segre [(21)] has been addressed by [25]. In this work, Kruchkovich contrastively obtained the following four classes of metrics

$$
\begin{aligned}
\mathrm{d} s^{2} & =2(2-c) e^{c x_{1}} \mathrm{~d} x_{1} \mathrm{~d} x_{2}+e^{2 x_{1}} \mathrm{~d} x_{3}^{2}, \quad c \neq 1,2, \\
\mathrm{~d} s^{2} & =e^{2 x_{1}}\left(2 \mathrm{~d} x_{1} \mathrm{~d} x_{2}-\mathrm{d} x_{3}^{2}\right), \\
\mathrm{d} s^{2} & =e^{-q x_{1}}\left[2 \mathrm{~d} x_{1} \mathrm{~d} x_{2}-\frac{4}{\omega^{2}} \cos ^{2}\left(\frac{\omega x_{1}}{2}\right) \mathrm{d} x_{3}^{2}\right]+k \mathrm{~d} x_{1}^{2}, \quad \omega=\sqrt{4-q^{2}}, \quad q^{2}<4, \\
\mathrm{~d} s^{2} & =e^{2 x_{3}} \mathrm{~d} x_{1}^{2}+2 n e^{x_{3}} \mathrm{~d} x_{1} \mathrm{~d} x_{2}+\varepsilon \mathrm{d} x_{3}^{2}, \quad n \neq 0, \quad \varepsilon= \pm 1 .
\end{aligned}
$$


However, this classification turns out to be redundant and consistent with our results. Indeed, one can bring the metrics (B.47a))-(B.47c) into a universal form:

$$
\mathrm{d} s^{2}=2 \mathrm{~d} x \mathrm{~d} y+\frac{a_{0}+a_{2} z^{2}}{x^{2}} \mathrm{~d} x^{2} \pm \mathrm{d} z^{2}
$$

where $a_{0}$ and $a_{2}$ are constants. This is nothing but the spacetime (B.31) up to the metric signature. The desired coordinate transformations are: For the metric (B.47a), plus sign in eq. (B.48) with

$$
\begin{aligned}
x_{1} & =\frac{1}{c} \log \left(\frac{c x}{2-c}\right), & x_{2} & =y+\frac{z^{2}}{2 c x}, \\
a_{2} & =\frac{1-c}{c^{2}}, & a_{0} & =0 .
\end{aligned}
$$

For the metric (B.47b), the minus sign in eq. (B.48) with

$$
x_{1}=\frac{1}{2} \log (2 x), \quad x_{2}=y-\frac{z^{2}}{4 x}, \quad x_{3}=\frac{z}{\sqrt{2 x}}, \quad a_{2}=\frac{1}{4}, \quad a_{0}=0 .
$$

For the metric (B.47c), the minus sign in eq. (B.48) with

$$
\begin{aligned}
& x_{1}=-\frac{1}{q} \log (q x), \quad x_{3}=\frac{\sqrt{4-q^{2}} z}{2 \sqrt{q x}} \sec \left(\frac{\sqrt{4-q^{2}}}{2 q} \log (q x)\right), \quad a_{0}=\frac{k}{q^{2}}, \\
& x_{2}=-y+\frac{z^{2}}{4 q x}\left[q-\sqrt{4-q^{2}} \tan \left(\frac{\sqrt{4-q^{2}}}{2 q} \log (q x)\right)\right], \quad a_{2}=\frac{1}{q^{2}} .
\end{aligned}
$$

It follows that the metrics admitting $4 \mathrm{KVs}$ in Segre [(21)] type are classified into two: one is the pp-wave (B.31) and the other is the plane-wave (B.44), both of which are homogeneous. This refines the analysis in [25].

\section{References}

[1] F. C. Klein, A comparative review of recent researches in geometry, arXiv:0807.3161 [math-DG].

[2] J. B. Zuber, Invariances in Physics and Group Theory, IRMA Lect. Math. Theor. Phys. 23, 307 (2015) doi:10.4171/148-1/12 [arXiv:1307.3970 [hep-th]].

[3] G. Darboux, Leçons sur la théorie générale des surfaces et les applications géométriques du calcul infinitésimal, III partie, Gauthier-Villars, Paris (1894).

[4] L.P. Eisenhart, A treatise on the differential geometry of curves and surfaces, Ginn and Company, Boston (1909).

[5] I. M. Singer, Infinitesimally homogeneous spaces, Comm. Pure Appl. Math. 13, 685-697 (1960).

[6] K. Nomizu, On Local and Global Existence of Killing Vector Fields, Ann. Math. 72, no.1, 105-120 (1960).

[7] F. Prüfer, F. Tricerri, L. Vanhecke, Curvature invariants, differential operators and local homogeneity, Trans. Amer. Math. Soc. 348, no. 11, 4643-4652 (1996).

[8] S. Console, C. Olmos, Curvature invariants, Killing vector fields, Connections and Cohomogeneity, Proc. of A.M.S. 137, no.10, 1069-1072 (2008).

[9] E. Cartan, Leçon sur la géometrie des Espaces de Riemann, II ed., Paris (1946).

[10] A. Karlhede, M. A. H. MacCallum, Gen. Rel. Grav. (1982) 14: 673. https://doi.org/10.1007/BF00761458 
[11] H. Stephani, D. Kramer, M. A. H. MacCallum, C. Hoenselaers and E. Herlt, Exact solutions of Einstein's field equations, doi:10.1017/CBO9780511535185

[12] B. Kruglikov and K. Tomoda, A criterion for the existence of Killing vectors in 3D, Class. Quant. Grav. 35, no. 16, 165005 (2018) doi:10.1088/1361-6382/aad11d [arXiv:1804.11032 [gr-qc]].

[13] R.P. Kerr, Scalar invariants and groups of motions in a $V_{n}$ with positive definite metric tensor, Tensor 12, 74-83 (1962).

[14] T. Houri and Y. Yasui, A simple test for spacetime symmetry, Class. Quant. Grav. 32, no. 5, 055002 (2015) doi:10.1088/0264-9381/32/5/055002 [arXiv:1410.1023 [gr-qc]].

[15] B. Kruglikov, The gap phenomenon in the dimension study of finite type systems, arXiv:1111.6315 [mathDG].

[16] B. O'Neill, The Geometry of Kerr Black Holes, A K Peters, Ltd., Wellesley, Massachusett, 1995.

[17] J. Santos, M. J. Rebouças, A. F. F. Teixeira, Gen Relat Gravit (1995) 27: 989. https://doi.org/10.1007/BF02113081

[18] S. Kachru, X.Liu, M. Mulligan, Gravity duals of Lifshitz-like fixed points, Phys. Rev. D78, 106005 (2008) doi:10.1103/PhysRevD.78.106005 [arXiv:0808.1725[hep-th]].

[19] H. Weyl, The classical groups, Princenton University Press (1946).

[20] A. Koutras, C. McIntosh, A metric with no symmetries or invariants, Class. Quantum Grav. 13, 4749 (1996).

[21] A. Coley, R. Milson, V. Pravda, A. Pravdová, Vanishing scalar invariant spacetimes in higher dimensions, Class. Quantum Grav. 21, 5519-5542 (2004).

[22] A. Coley, S. Hervik, N. Pelavas, Spacetimes characterized by their scalar curvature invariants, Class. Quantum Grav. 26, 025013 (2009).

[23] M. Banados, C. Teitelboim and J. Zanelli, The Black hole in three-dimensional space-time, Phys. Rev. Lett. 69 (1992) 1849 [hep-th/9204099].

[24] M. Banados, M. Henneaux, C. Teitelboim and J. Zanelli, Geometry of the (2+1) black hole, Phys. Rev. D 48 (1993) 1506 Erratum: [Phys. Rev. D 88 (2013) 069902] [gr-qc/9302012].

[25] G. I. Kruchkovich, Invariant criteria of spaces $V_{3}$ with the group of motions $G_{4}$, Uspekhi Mat. Nauk, 10:1(63) (1955), 129-136. To the paper "Classification of three-dimensional Riemannian spaces according to groups of motions”, Uspekhi Mat. Nauk, 9:3(61) (1954), 285.

[26] D. Klemm and M. Nozawa, Geometry of Killing spinors in neutral signature, Class. Quant. Grav. 32 (2015) no.18, 185012 [arXiv:1504.02710 [hep-th]]. 